\title{
Recherches écologiques sur les faunes des plages arbitées de la région d'Arcachon ${ }^{1}$
}

\author{
M. Amanieu \\ Institut de Biologie marine de l'Université de Bordeaux, \\ Arcachon, Gironde, France
}

\begin{abstract}
Ecological research on the faunas of the sheltered beaches of the region of Arcachon. In these ecological studies of the sheltered beaches of the bay of Arcachon (France), the author distinguishes and locates four traditional zones: slikke, upper slikke, schorre and parhalian zone. Animal communities and edaphic structures of each zone are treated in detail. In the slikke, four fundamental facies are distinguished: Zostera nana zones, bare sands (such as Arenicola sands), Nereis diversicolor slime and Scrobicularia slime. Each facies is analyzed with regard to its amount of water, porosity, salinity, $\mathrm{pH}$ and dissolved oxygen content, as well as its hypsometric distribution. With respect to the fauna of sand and slime slikkes, the importance of the endogeous macrofauna, its infralittoral characteristics and the low degree of mobility of its species are emphasized. In the upper slikke five facies are described: Bledius spectabilis clay sands, Coropbium volutator slimy sands, Bledius arenarius porous sands, Spartina maritima areas and micro cliffs. Again, abiotic characteristics and animal communities of each facies are studied successively. The importance of edaphic structures for the distribution of animal communities, and vice versa, is stressed. For instance, the amphipod Coropbium volutator and the coleopteran Bledius spectabilis build tubes with firm and permanent inner surfaces only in certain types of sediment: yet, while the Bledius spectabilis tubes are dense and watertight, those of Corophium volutator are less tight and often flooded. Thus, the presence of the insect allows a supralittoral fauna to establish itself, whereas that of the amphipod is, in contrast, accompanied by a mediolittoral fauna resistant to flooding. In the schorre and parhalian zone, endogeous macrofauna elements are entirely absent; the vagrant epigeous species exhibit considerable degrees of mobility, migrating according to tidal rhythms. In summer, the schorre zone shelters both marine supralittoral or halobiotic species as well as adlittoral "haloxene" species which, coming from the parhalian zone, are attracted by the trophically rich Puccinellietum. In winter, however, frequent floodings of the schorre force the fauna to retreat to the parhalian. zone for shelter. A classification is presented of the facies studied, based on the amount of water ecologically usable by their respective macrofaunas. The relationships between slikke, upper slikke, schorre and parhalian zone on the one hand, and the intertidal zones on the other are illustrated in the form of a synopsis. Finally, a comparison is made between animal communities of sheltered and of partly sheltered beades.
\end{abstract}

${ }^{1}$ Ce travail constitue la 2ème partie d'une thèse de Doctorat ès Sciences naturelles soutenue à la Faculté des sciences de l'Université de Bordeaux (no d'ordre 197) et intitulée «Recherches écologiques sur les faunes des plages arbitées et des étangs saumatres de la region d'Arcachon». L'exemplaire dactylographié de la thèse in extenso a été déposé au C.N.R.S. et enregistré sous le no A.O. 1612. 


\section{CARACTERES GENERAUX DES PLAGES ABRITEES}

\section{Slikkes et schorres}

L'originalité des diverses plages d'une côte tient, notamment, à un ensemble de conditions abiotiques dans lesquelles, depuis fort longtemps, on a coutume de distinguer trois grands ordres de phénomènes; la nature du substratum ou faciès (РRUvoт 1897, faciès rocheux ou sableux); l'exposition à l'océan ou mode (DE BeAuchamp 1914, mode abrité ou exposé); les caractéristiques hydrologiques ou régime (Monon 1926, régime marin ou saumâtre).

Dans la région d'Arcachon, le mode permet de reconnaître trois types de plages: les plages océaniques (côte ouest du Cap-Ferret, littoral au sud de Pineau), les plages semi-abritées (côte est du Cap-Ferret, est du banc d'Arguin, littoral entre les Abatilles et Pyla sur mer), les plages abritées (pourtour de la baie à l'est et au nord-est d'Arcachon, pourtour de l'Ile aux Oiseaux).

Les plages abritées, auxquelles est consacré le présent travail, sont caractérisées par la tranquilité des eaux qui les recouvrent. Cette tranquilité s'accompagne, lorsque le sédiment est meuble, comme c'est le cas à Arcachon, d'une part d'une augmentation de la fraction fine dans le matériel sédimenté, d'autre part d'un allongement souvent considérable des dimensions de l'estran.

Massart (1907/1908) a introduit dans la description des plages abritées, particulièrement dans les régions d'estuaires, les termes flamands de schorre et de slikke, dont la définition s'appuie sur deux critères distincts: d'une part un critère bathymétrique: la slikke est inondée à toutes les marées, le schorre seulement aux marées de vive eau; d'autre part un critère bionomique: la couverture végétale de la slikke est pauvre et généralement discontinue (algues, diatomées, herbiers de Zostera nana), alors qu'elle est dense et continue sur le schorre (phanérogames halophiles dressées).

De Beauchamp (1914) et Sollaud (1925) appliquèrent les notions de slikke et de schorre aux estuaires de la Penzé (Roscoff) et de la Slack (Ambleteuse), dans le sens défini par Massart. Monod (1926), dans un travail classique sur la Basse Seule, examine de manière beaucoup plus attentive la définition initiale de l'auteur belge et met très justement l'accent sur le fait que, d'une part, les critères bathymétrique et bionomique retenus par MASSART conduisent chacun à des définitions distinctes de la slikke et du schorre, d'autre part, sur l'intérêt essentiellement bionomique, beaucoup plus que bathymétrique, des notions introduites par cet auteur. C'est de même à une défnition morphologique, très voisine de la conception bionomique de Monod, que s'arrête Francis-BOEUf (1947).

\section{Haute slikke}

JACQUET (1949) à propos d'un travail portant sur l'extension de la spartine de Townsend dans la Manche, est amené à réexaminer le critère bathymétrique de MAsSART. Il souligne que, dans le Havre de Regnéville par exemple, les marées hautes de morte eau culminent à $+9,20 \mathrm{~m}$, tandis que la bordure externe du pré salé ne descend pas au-dessous de $12,82 \mathrm{~m}$, se terminant par un abrupt de 1 mètre environ, dont la 


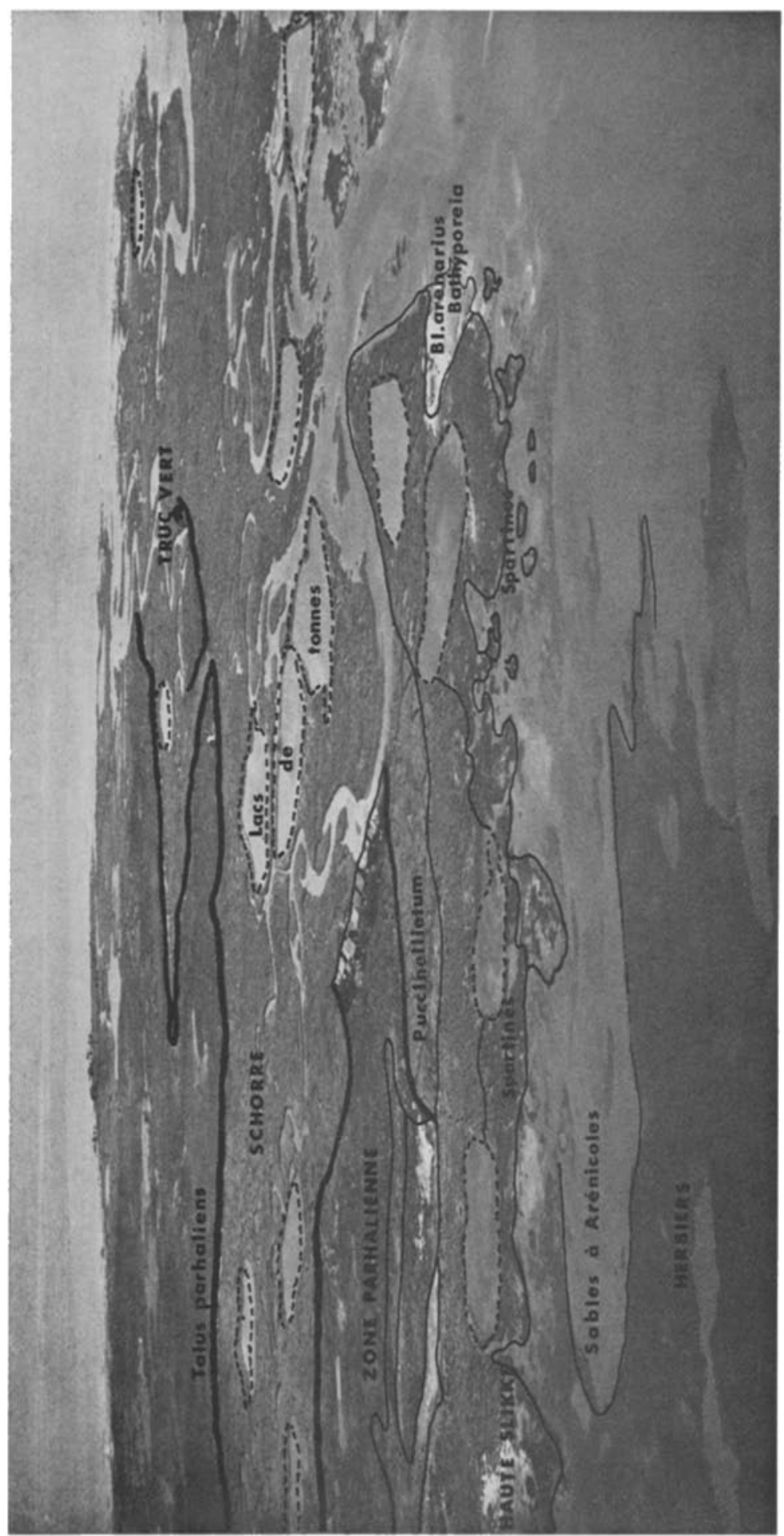

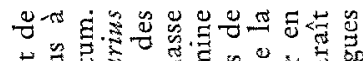

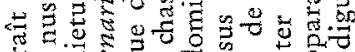


능. त.

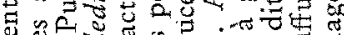
过 0

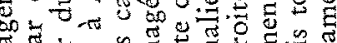

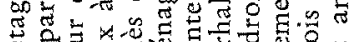


볼 告 于

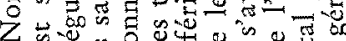

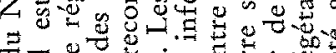

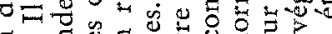



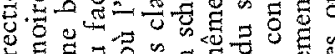

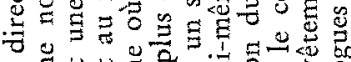



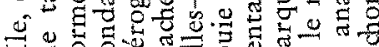


षै \&

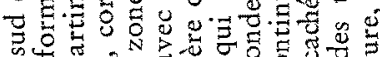
कै

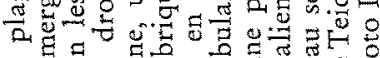


क षे जै

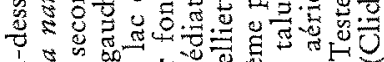

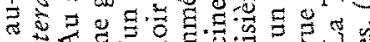

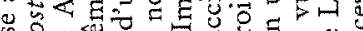

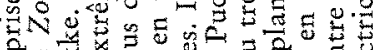

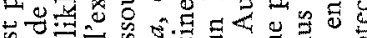
它 \% ข इंड ช

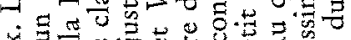
F के

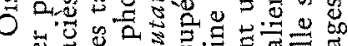

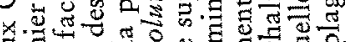
है

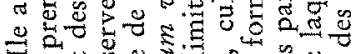

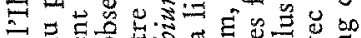

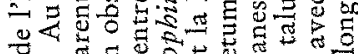

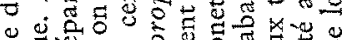

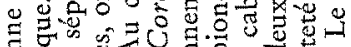
.

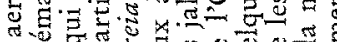

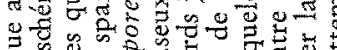
$>$ of

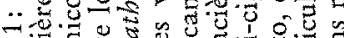




base se situe donc à la cote moyenne de $11,82 \mathrm{~m}$. JACQUET est ainsi amené à individualiser un troisième niveau, qu'il nomme «haute slikke», intercalé topographiquement entre la slikke et le schorre. La notion de haute slikke dèpasse le seul intérêt topographique et correspond à une unité bionomique sur laquelle nous reviendrons.

\section{Limites externes des slikkes et des schorres. Zone parhalienne}

Du côté inférieur, la slikke est limitée sans conteste au bord du chenal ou lit mineur de la rivière. Elle inclut, lorsqu'il existe, l'herbier de Zostera nana (Massart 1907/1908).

Du côté supérieur, la limite continentale du schorre est beaucoup plus difficile à établir. Francis-Boevf (1947), considère le schorre comme un ensemble morphologique, d'origine géologique, pouvant inclure des terrains qui ne sont plus actuellement inondables par la marée, par exemple les ouldjas cultivables de l'estuaire du BouRegreg au Maroc. Comme l'a souligné JACQUET (1949), si cette opinion est soutenable aux regards d'un géographe, il ne saurait en être de même à ceux d'un écologiste. VERGER (1956) a très justement opposé les schorres littoraux aux schorres d'estuaires. Dans les schorres littoraux, le critère bathymétrique coincide avec la marée de salinité; un accident topographique, digue, cordon littoral, roche en place, marque topographiquement la limite des hautes eaux; cet accident s'accompagne alors de la présence d'un niveau bionomique original de faible extension, la zone parhalienne, qui marque nettement le passage des formations halophiles aux formations continentales. Dans les schorres d'estuaires, la rupture morphologique de la pente de la plage, lorsqu'elle existe, est généralement moins accusée; en outre, les fortes marées s'accompagnent encore d'inondations, mais l'eau recouvrante est une eau presque douce, provenant des débordements de la rivière, qui vient buter sur la haute barrière des eaux marines. Dans le domaine littoral, la limite des eaux coincidant avec la marée de salinité, il n'y a aucun inconvénient à s'appuyer sur le critère bathymétrique, d'appréciation plus aisée. Au fond de l'estuaire au contraire, seul le critère hydrologique à un sens mais, loin d'être nettement limité comme le précédent, il évolue ici d'une manière continue, le long d'une frange plus ou moins vaste où toute définition se dilue.

JACQUET (1949) définit la zone parhalienne comme «une région semi-halophile, non inondable et qui ne reçoit que les embruns». En fait, dans les plages abritées, les embruns sont rares et de faible amplitude. La zone parhalienne correspond alors non plus à une zone d'embruns mais aux niveaux $d$ ' inondation des hautes mers hivernales exceptionnelles, à Arcachon, au de là de $+4,60$ m environ (Fig. 1).

\section{LA SLIKKE}

\section{Introduction}

Nous aborderons en premier lieu l'étude de la slikke. En ce qui concerne l'hydrologie des eaux recouvrantes nous renvoyons au travail de Amanieu (1966). Ici même nous étudierons les conditions édaphiques et les peuplements. 
Nous reconnaîtrons dans les slikkes de la baie d'Arcachon quatre ensembles fondamentaux qui ont valeur de faciès: (1) les herbiers de Zostera nana, (2) les sables à Arénicoles, (3) les vases à Nereis diversicolor et (4) les vases à Scrobicularia plana. Les conditions édaphiques sont décrites séparément pour chacun des faciès. Les peuplements sont étudiés, d'une part sous la forme de listes faunistiques, d'autre part sous la forme de remarques systématiques et écologiques.

\section{Les herbiers de Zostera nana}

Les herbiers de Zostères du littoral français ont, depuis longtemps, fait l'objet de remarquables travaux de faunistique. Plus récemment ont été abordés les problèmes écologiques et biocénotiques (Peres \& Picart 1955 et 1964, Lubet 1956, Blois et al. 1961, Ledoyer 1962 et 1964, True Schenlz 1965) ou pédologiques (Deguen \& Molinier 1961, Blots et al. 1961).

\section{Herbiers d'Arcacbon - Teneur en eau}

La teneur en eau des sédiments est déterminée par la perte de poids d'un échantillon frais après passage à l'étuve à $110^{\circ}$ jusqu'à poids constant $(24 \mathrm{~h}$ en moyenne). Elle est exprimée ici par rapport à $100 \mathrm{~g}$ de sédiment sec. Six herbiers repérés sur la carte Figure 2 ont été explorés. Sur chaque herbier 20 échantillons, de $100 \mathrm{~g}$ chacun en moyenne, ont été prélevés en quinconce à 1 mètre de distance les uns des autres.

Tableau 1

Teneur en eau des sols d'herbiers (Arcachon 1965)

\begin{tabular}{|ccccccc|}
\hline Localités & Janvier & Avril & Août & Octobre & Moyenne & $\begin{array}{c}\text { Plus grand } \\
\text { ecart }\end{array}$ \\
\hline $\begin{array}{l}\text { La Hume } \\
0-5 \mathrm{~cm}\end{array}$ & 54 & 48 & 38 & 48 & 47 & 16 \\
$-10-15$ & 33 & 32 & 30 & 31 & 31,5 & 3 \\
$\begin{array}{l}\text { Cassy } \\
0-5 \mathrm{~cm}\end{array}$ & 165 & 165 & 134 & 183 & 162 & 49 \\
$-10-15$ & 39 & 42 & 38 & 45 & 41 & 7 \\
$\begin{array}{l}\text { Taussat } \\
0-5 \mathrm{~cm}\end{array}$ & 139 & 127 & 116 & 139 & 130 & 23 \\
$-10-15$ & 43 & 41 & 41 & 41 & 41,5 & 2 \\
$\begin{array}{l}\text { Andernos } \\
0-5 \mathrm{~cm}\end{array}$ & 228 & 209 & 215 & 203 & 214 & 25 \\
$-10-15$ & 39 & 41 & 33 & 32 & 36 & 9 \\
$\begin{array}{l}\text { Lège } \\
0-5 \mathrm{~cm}\end{array}$ & 245 & 201 & 245 & 217 & 227 & 44 \\
$-10-15$ & 124 & 118 & 128 & 121 & 123 & 10 \\
$\begin{array}{l}\text { Ile } \\
0-5 \mathrm{~cm}\end{array}$ & 99 & 98 & 95 & 133 & 106 & 38 \\
$-10-15$ & 32 & 35 & 32 & 35 & 33,5 & 3 \\
\hline
\end{tabular}


Pour chaque échantillon, j'ai mesuré séparément la teneur en eau des strates respectivement comprises entre 0 et $-5 \mathrm{~cm}$, puis entre -10 et $-15 \mathrm{~cm}$. La séparation de ces deux strates est effectuée sur place: 1 manchon métallique de $12 \mathrm{~cm}$ de diamètre est enfoncé verticalement dans le sol à marée basse; les strates des différents niveaux sont récoltées avec une petite pelle à l'intérieur du manchon. Les teneurs en eau de chaque herbier concernent la moyenne des vingt échantillons récoltés dans chaque station. Les résultats sont indiqués dans le Tableau 1. Ce tableau fait ressortir la présence quasi constante, sauf à la Hume, d'une strate superficielle largement sursaturée, qui inclut une couche de vase thixotrophique, à très haute teneur en eau. Cette strate souligne l'originalité des herbiers en mode très calme de la baie d'Arcachon. Par opposition, la teneur en eau de la strate profonde apparaît comme moins élevée et plus stable.

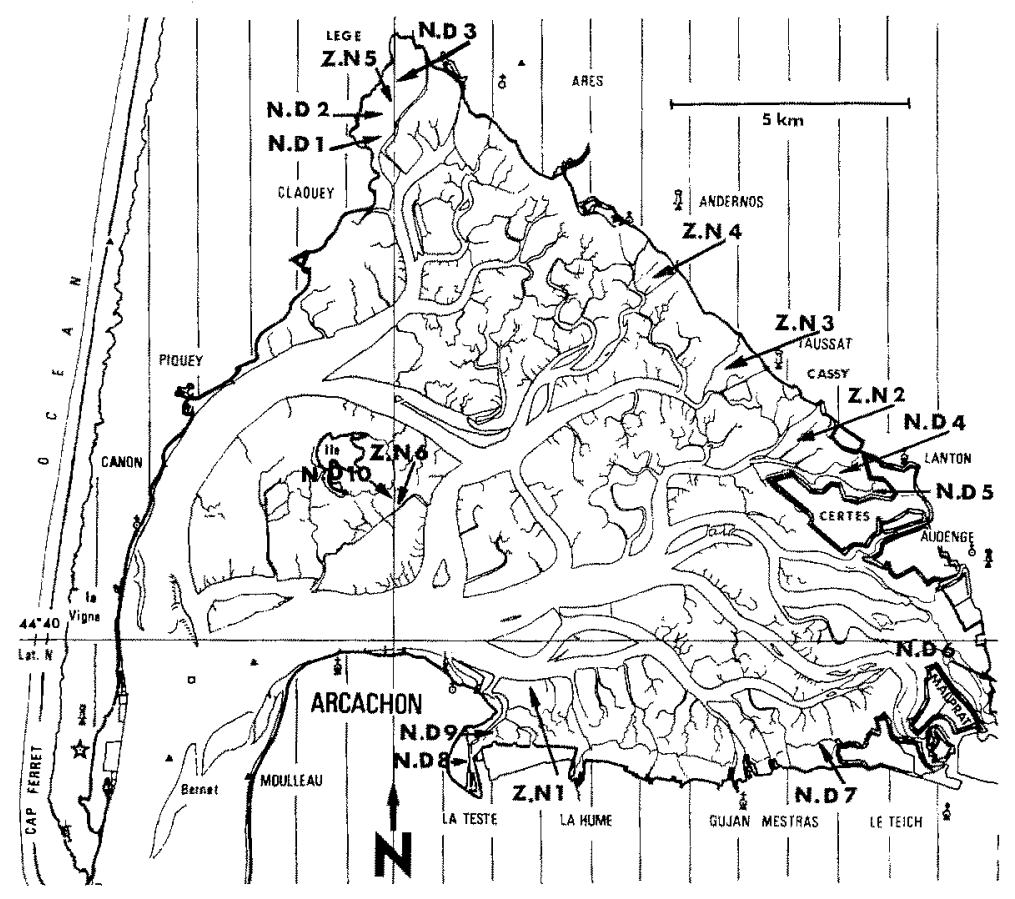

Fig. 2: Emplacement des stations prospectées dans la slikke. ZN 1 à $6=$ herbiers de Zostera nana. Les sables à arénicoles étudiés sont contigus à ces herbiers. ND 1 à $10=$ Vases à Nereis diversicolor

\section{Salinité des eaux interstitielles}

J'ai étudié comparativement une station à Lège (sud de la dune de Lillan) et l'autre sur l'Ile aux Oiseaux. Les moyennes mensuelles sont calculées à partir de 1 à 3 prélèvements hebdomadaires. L'eau dite des flaques est directement récoltée à même le sol. L'eau interstitielle est extraite mécaniquement du sédiment, en le comprimant entre les mâchoires d'un presse fruit. Les résultats sont rassemblés graphiquement 
Tableau 2

Salinités des eaux interstitielles des herbiers de l'lle aux oiseaux et de Lège (en 1962)

\begin{tabular}{|c|c|c|c|c|c|c|c|}
\hline \multicolumn{2}{|c|}{ Eyrac eau du Chenal } & \multicolumn{2}{|c|}{$\begin{array}{l}\text { Minimum } \\
\text { Annuel }\end{array}$} & \multicolumn{2}{|c|}{$\begin{array}{c}\text { Maximum } \\
\text { Annuel }\end{array}$} & $\begin{array}{r}\text { Ecart } \\
5,33\end{array}$ & $\begin{array}{c}\text { Moyenne } \\
\text { Annuelle } \\
31,67\end{array}$ \\
\hline $\begin{array}{l}\text { Ile aux } \\
\text { Oiseaux }\end{array}$ & $\begin{array}{l}\text { Flaques } \\
\text { Strate 0- } 5 \mathrm{~cm} \\
\text { Strate } 10-15 \mathrm{~cm}\end{array}$ & $\begin{array}{l}\text { Avril } \\
\text { Février } \\
\text { Avril }\end{array}$ & $\begin{array}{l}26,0 \\
26,5 \\
27,5\end{array}$ & $\begin{array}{l}\text { Août } \\
\text { Septembre } \\
\text { Septembre }\end{array}$ & $\begin{array}{l}39,5 \\
38,0 \\
33,0\end{array}$ & $\begin{array}{r}13,5 \\
11,5 \\
5,5\end{array}$ & $\begin{array}{l}31,4 \\
32,08 \\
30,50\end{array}$ \\
\hline $\begin{array}{l}\text { I.ège } \\
\text { (Lillan) }\end{array}$ & $\begin{array}{l}\text { Strate } 0-5 \mathrm{~cm} \\
\text { Strate } 10-15 \mathrm{~cm}\end{array}$ & $\begin{array}{l}\text { Mars } \\
\text { Janvier }\end{array}$ & $\begin{array}{l}15,5 \\
19,5\end{array}$ & $\begin{array}{l}\text { Août } \\
\text { Septembre }\end{array}$ & $\begin{array}{l}30,5 \\
25,0\end{array}$ & $\begin{array}{r}15,0 \\
5,5\end{array}$ & $\begin{array}{l}22,6 \\
21,95\end{array}$ \\
\hline
\end{tabular}

Figure 3 et résumés sur le Tableau 2. On note, dans les deux stations, que la salinité de la strate profonde est beaucoup plus stable que celle des strates superficielles. En outre, à Lège, l'ensemble du sédiment est imprégné d'une eau beaucoup plus dessalée que sur l'Ile aux Oiseaux.

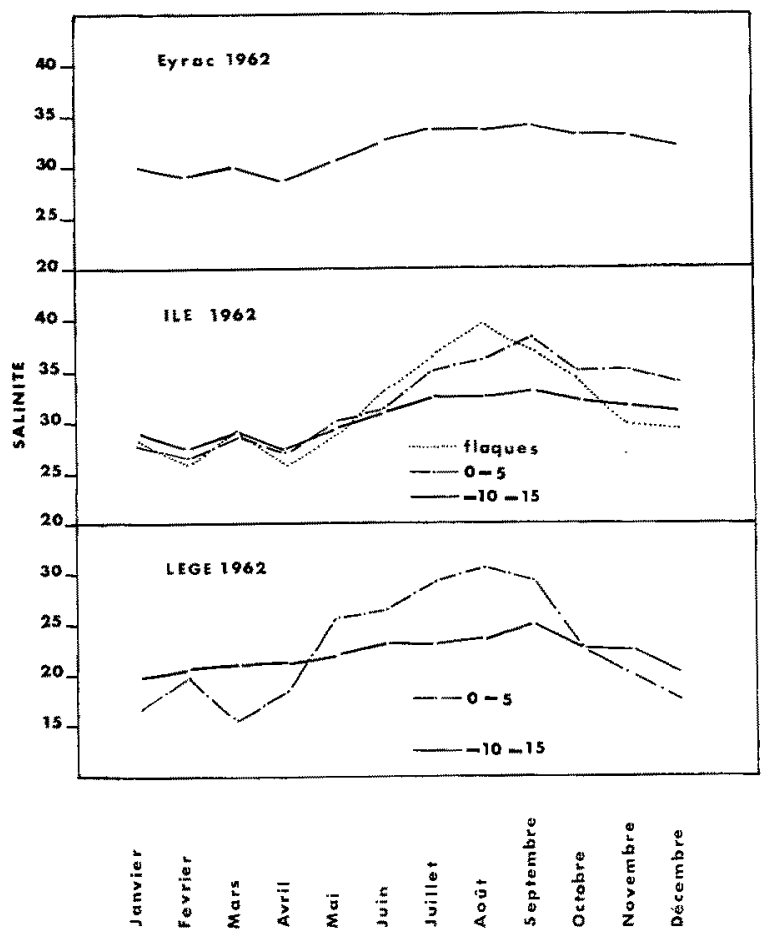

Fig. 3: Graphique des variations saisonnières de salinité (\%) dans les herbiers de Lège et de l'Íle aux Oiseaux. Comparaison avec les eaux du Teychan à Eyrac

Sur l'Ile aux Oiseaux on remarque que la salinité de la strate superficielle suit étroitement les variations de celle des flaques de janvier à août, c'est à dire pendant la période où la salinité croît régulièrement dans les flaques. D'août à septembre, la 
salinité decroît dans les flaques, mais continue à croître dans la strate superficielle. Durant l'été, la strate superficielle s'imprègne des eaux de ruissellement, sursalées par suite de l'évaporation, leur densité élevée favorisant alors leur pénétration. En revanche, lorsque la salinité des flaques décrôtr, les échanges avec l'eau interstitielle sont alors plus difficiles; l'eau qui imprègne le sédiment superficiel provient non plus de l'eau des flaques légère et de faible épaisseur, mais de l'eau recouvrante apportée par la marée haute, dont la pression facilite l'intrusion.

A Lège la salinité des flaques est trop fluctuante pour permettre l'établissement de moyennes significatives. La salinité de la strate superficielle supporte elle même de grands écarts saisonniers (de 15,5\% en mars à 30,5\% en août). La salinité de la strate profonde est plus stable mais sa moyenne reste basse $(21,95 \%)$.

\section{pH et oxygène dissous}

Les mesures effectuées le 17 juillet 1965 (marée de coefficient 69, basse mer à $13.42 \mathrm{~h}$ ) sur les herbiers du sud de l'lle aux Oiseaux, donnent des indications sur la variation de ces deux facteurs au cours d' une marée (Tab. 3). L'oxygène dissous a été dosé par la méthode de WinkLer. Les mesures de $\mathrm{pH}$ ont été faites in situ, à l'aide d'un $\mathrm{pH}$ mètre portatif Métrohm à piles. J'ai comparé les données recueillies sur l'herbier, à celles qui concernent les eaux du chenal du Teychan voisin.

Au dessus de l'herbier, l'eau est à la fois plus alcaline, plus oxygénée, plus chaude et plus salée que dans le chenal, ensemble de conditions liées à la forte insolation dont bénéficient les herbiers depuis le matin.

A marée basse, on doit distinguer sur l'herbier, d'une part l'eau superficielle, qui se collecte librement dans les dépressions et forme des flaques, d'autre part l'eau de gravitation, qui exsude lentement au niveau des «dégoulinants», terme local imagé, désignant les sources temporaires formées lors du tassement de l'herbier après le retrait de la mer. L'eau des flaques est alcaline et oxygénée (photosynthèse), inversement l'eau

Tableau 3

Mesures de $\mathrm{pH}$ et oxygène dissous (Ile aux Oiseaux)

\begin{tabular}{|c|c|c|c|c|}
\hline Région & $\mathrm{pH}$ & $\begin{array}{l}\mathrm{O}_{2} \text { dissous } \\
(\mathrm{mg} / \mathrm{l})\end{array}$ & $\begin{array}{c}\text { Température } \\
\left({ }^{\circ} \mathrm{C}\right)\end{array}$ & $\begin{array}{c}\text { Salinité } \\
(\% 0)\end{array}$ \\
\hline $\begin{array}{l}\text { (A) Entre } 12 \mathrm{~h} \text { et } 12.30 \mathrm{~h} \\
\text { Milieu du chenal } \\
\text { Sur l'herbier ( } 30 \mathrm{~cm} \text { du sol) }\end{array}$ & $\begin{array}{l}8,2 \\
8,4\end{array}$ & $\begin{array}{r}7,55 \\
10,25\end{array}$ & $\begin{array}{l}22^{0} \\
24^{0}\end{array}$ & $\begin{array}{l}33,0 \\
33,2\end{array}$ \\
\hline $\begin{array}{l}\text { (B) Entre } 13.40 \text { h et } 14 \mathrm{~h} \text { (Etale de } \\
\text { Milieu du chenal } \\
\text { Flaques stagnantes sur l'herbier } \\
\text { Eeau qui sourd d'un «dégoulinant» }\end{array}$ & $\begin{array}{r}\text { mer) } \\
8,2 \\
8,9 \\
6,8\end{array}$ & $\begin{array}{c}7,58 \\
18,7 \\
3,2\end{array}$ & $\begin{array}{l}22^{\circ} \\
24^{\circ} \\
24^{0}\end{array}$ & $\begin{array}{l}33,0 \\
33,8 \\
33,9\end{array}$ \\
\hline $\begin{array}{l}\text { (C) Entre } 15 \text { h et } 15.15 \text { h (Flot) } \\
\text { Milieu du chenal } \\
\text { Nappe du flot recouvrant l'herbier } \\
\text { Zones où l'herbier est déjà sous } \\
30 \mathrm{~cm} \text { d'eau }\end{array}$ & $\begin{array}{l}8,2 \\
6,9 \\
8,1\end{array}$ & $\begin{array}{l}8,0 \\
4,6 \\
8,0\end{array}$ & $\begin{array}{l}22^{0} \\
23,3^{0} \\
22,2^{0}\end{array}$ & $\begin{array}{l}33,1 \\
33,8 \\
33,2\end{array}$ \\
\hline
\end{tabular}


d'imprégnation est très acide et peu oxygénée (action du sol). A marée basse, les grands herbiers plats de Zostera nana sont recouverts d'une mince nappe d'eau, qui est en réalité non pas l'eau résiduelle de la marée, mais l'eau interstitielle qui exsude du sédiment.

Ces relevés font ressortir que le flot refoule devant lui la nappe acide et réduite de l'eau de gravitation qui suinte pendant la basse mer, et la remplace très rapidement par une nappe plus alcaline et plus oxygénée, qui vient du chenal. Plus lentement ensuite, durant toute la marée haute de jour, les caractéristiques hydrologiques évoluent vers une plus grande oxygénation, s'accompagnant d'une alcalinisation. En fin de marée haute, la teneur en oxygène dissous est en effet passée à 11,3 $\mathrm{mg} / 1$ (temps couvert) et le $\mathrm{pH}$ à 8,7 ; cette évolution est évidemment en relation avec l'activité photo synthètique des zostères.

\section{Répartition bypsométrique}

Zostera nana couvre, au nord d'une ligne Arcachon Bélisaire, la majeure partie de la portion inférieure des plages sablo-vaseuses de la Baie. Selon Boucher (1962b), la limite inférieure de Zostera nana coïncide généralement avec la limite supérieure de Zostera marina et la courbe de niveau $+0,30 \mathrm{~m}$ environ. Les cotes indiquées ici, sont toujours exprimées par rapport au zéro des cartes marines locales. A partir de repères qui m'ont été indiqués par M. Beaupuy et par M. Bordenave, Ingénieur à l'I.G.N., que je remercie vivement, j'ai nivelé en 408 points, répartis autour de la Baie, la bordure supérieure des différents herbiers. Les resultats sont rassemblés çi-dessous.

La Teste: $+2,86 \mathrm{~m}$ au contact de vases.

La Hume: $+2,40 \mathrm{~m} \grave{a}+2,80 \mathrm{~m}$ au contact de sables à arénicoles.

Mestras: $+2,70 \mathrm{~m} \grave{a}+2,85 \mathrm{~m}$ au contact de sables vaseux envahis par des spartines.

Malprat: au nord $+2,80 \mathrm{~m}$ au contact de sables vaseux envahis par des partines, au sud $+3,12 \mathrm{~m}$ l'herbier remonte lorsque l'on avance dans l'estuaire.

Certes: au nord, baie de Lanton, $+3,05 \mathrm{~m}$, au contact de vases à Nereis diversicolor; au sud, $+2,40 \mathrm{~m}$, au contact de sables propres et bien égouttés.

Lège: entre $+2,60 \mathrm{~m}$ et $+2,80 \mathrm{~m}$, au contact de sables ou de vases variés.

Ile aux Oiseaux: plage sud, $+1,95 \mathrm{~m}$ à $+2,45 \mathrm{~m}$, au contact des sables à arénicoles, plage est, $+2,00 \mathrm{~m}$ à $+2,10 \mathrm{~m}$, au contact de sables variés, plage nord, $+2,55 \mathrm{~m}$ $\grave{a}+2,65 \mathrm{~m}$, au contact de sables vaseux. $+2,45 \mathrm{~m}$ à $+3,05 \mathrm{~m}$, dans les dépressions ou le long des rives d'esteys.

\section{Remarques}

(1) Les nivellements rapportés concernent les mesures faites durant l'été 1963.

(2) Malprat, Certes, et l'Ile aux Oiseaux appelent des renseignements complémentaires.

Malprat: A la pointe nord de l'lle, l'herbier vient au contact de sables vaseux envahis par des spartines et culmine à $+2,80 \mathrm{~m}+2,85 \mathrm{~m}$. Le long de la digue ouest, 
les apports de la Leyre accentuent la dessalure, les spartines sont remplacées progressivement par Juncus maritimus, puis, plus encore en amont de la rivière, par Scirpus maritimus (digue sud). L'herbier s'arrête vers le sud, au niveau de l'extrême pointe des réservoirs de la Saussouze (dits de Bayonne). En longeant la digue ouest, du nord vers le sud, lherbier remonte progressivement de plus en plus haut sur la pente de la plage, de la cote précédente $+2,80 \mathrm{~m}$ à la cote $+3,12 \mathrm{~m}$; sa frange supérieure ne suit donc pas une courbe de niveau, mais au contraire s'élève progressivement, au fur et à mesure que l'on s'avance dans l'estuaire de la rivière.

Certes: Les réservoirs du domaine de Certes sont entourés d'une longue digue qui s'avance profondément dans la baie vers l'ouest. A l'extrémité ouest (pointe de Branne), l'herbier de Zostera nana vient au contact des plus basses spartines, et culmine à la cote moyenne $+2,95 \mathrm{~m}$. Vers l'est, en pénétrant dans la petite baie de Lanton, l'herbier se dissocie des spartines, une bande de vases à Nereis diversicolor d'abord étroite, puis de plus en plus large à mesure que l'on se dirige vers Lanton, vient s'intercaler entre les spartines et les zostères. Simultanément, la bordure supérieure de l'herbier remonte très légèrement et culmine à $+3,00 \mathrm{~m},+3,05 \mathrm{~m}$ vers Lanton. En longeant au contraire la digue ouest des réservoirs, depuis la pointe de Branne jusqu'à l'entrée du port de Certes, ce ne sont plus des vases, mais des sables, qui viennent s'intercaler entre spartines et zostères; la limite supérieure de l'herbier s'abaisse progressivement, de la cote $+2,95 \mathrm{~m}$ (pointe de Branne), jusqu'à la cote $+2,40 \mathrm{~m}$. En définitive, la frange supérieure de l'herbier autour des digues de Certes, s'élève progressivement de la cote $+2,40 \mathrm{~m}$ (sud de la digue ouest), à la cote $+3,00 \mathrm{~m}$ (est de la digue nord).

Ile aux Oiseaux: Plage sud, l'herbier qui borde l'estey de Gahignon vient au contact de sables à arénicoles, le long d'une ligne très fluctuante qui oscille entre $+1,95 \mathrm{~m}$ et $+2,45 \mathrm{~m}$. Entre cet herbier et le schorre, on retrouve des zostères, installées dans des cuvertes, formant plus ou moins enclaves au milieu des sables à arénicoles, cuvettes dont les zostères occupent le fond, puis les bords, jusqu'à la cote $+2,70 \mathrm{~m}$. A l'est des cabanes Tchanquées, l'herbier qui borde le chenal culmine à $+2,00 \mathrm{~m},+2,10 \mathrm{~m}$; mais lorsque l'on remonte le petit estey qui conduit au Truc Vert, la limite supérieure des herbiers qui en tapissent les rives, s'élève progressivement jusqu'ì la cote $+2,45 \mathrm{~m}$. A l'est du puit artésien, plage nord, les herbiers qui bordent-le" chenal culminent à $+2,55 \mathrm{~m},+2,65 \mathrm{~m}$, mais au dela on retrouve des zostères dans des cuvettes formant enclaves, jusqu'à la cote $+3,05 \mathrm{~m}$.

(3) Ces chiffres montrent qu'ainsi que l'on pouvait s'y attendre, la limite supéricure de Zostera nana dans la baie d'Arcachon, est loin de se distribuer sur une courbe de niveau, puisqu'elle oscille entre les cotes extrêmes de $+1,95 \mathrm{~m}$ (plage sud de l'Ile aux Oiseaux) à $+3,12 \mathrm{~m}$ (dans l'estuaire de la Leyre). Cette limite est cependant nettement plus élevée que ne l'estime LubET (1956), qui situe le sommet de Zostera nana «au niveau des basses mers moyennes de mortes eaux: cote $+1,10 \mathrm{~m}$ par rapport au 0 des cartes marines (limite de l'étage méso-littoral et de l'étage infralittoral)».

(4) Parmi les cotes les plus basses, nous notons celles de la Hume, $+2,40 \mathrm{~m}$, Certes, $+2,40 \mathrm{~m}$, l'lle aux Oiseaux, $+1,95 \mathrm{~m}$. Dans tous ces cas, l'herbier vient au contact de sables propres bien égouttés, retenant peu d'eau à marée basse; incontestablement l'abaissement de la frange supérieure est alors lié à la bonne perméabilité du sédiment. 
(5) Parmi les cotes les plus élevées, nous notons celles de Malprat, $+3,12 \mathrm{~m}$, Certes (baie de Lanton), + 3,05 m, l'lle aux Oiseaux, $+3,05 \mathrm{~m}$. Dans tous ces cas, l'herbier vient au contact de vases qui restent imprégnées d'eau après le retrait de la mer, ou bien est installé dans des dépressions formant cuvettes.

(6) En définitive, l'herbier de Zostera nana culmine habituellement à $2,80 \mathrm{~m}$, $+3,00 \mathrm{~m}$ environ. De part et d'autre de cette valeur moyenne, la frange supérieure de l'herbier s'abaisse jusqu'à la cote $+1,95 \mathrm{~m}$ sur les plages bien égouttées à sédiment perméable: elle s'élève au contraire au delà de $3 \mathrm{~m}$, lorsque la nature du sol, ou la configuration du terrain, entrietiennent une humidité permanente, même après le retrait de la mer.

(7) La zone occupée par les herbiers de Zostera nana se situe en résumé entre $+0,30 \mathrm{~m}$ (Bouchet 1962b) et $+2,80 \mathrm{~m}$ environ; elle a donc une ampleur de $+2,50 \mathrm{~m}$, soit plus de la moitié de la zone intertidale. Zostera nana occupe la majeure partie de la moitié inférieure des plages abritées de la baie d'Arcachon. Bien qu'une partie des herbiers ait été aménagée pour l'installation des concessions ostréicoles, le faciès de la petite zostère couvre, dans l'ensemble de la baie, plusieurs milliers d'hectares.

\section{Les sables à arénicoles}

Situés topographiquement entre l'herbier de Zostera nana et la haute slikke, les sables à arénicoles de la région d'Arcachon sont surtout développés d'une part autour de l'Ile aux Oiseaux, d'autre part sur les plages de la Hume et de Lège. Vers le sud de la Baie, ils passent progressivement aux sables des plages semi-abritées, étudiées par Davant \& Salvat (1961). Vers le nord et vers l'est, ils passent au contraire aux vases à Nereis diversicolor.

\section{Teneur en eau}

Les sables à arénicoles se séparent en deux strates, une strate superficielle peu épaisse, oxygénée et de couleur claire, une strate profonde, foncée et réduite. La teneur en eau de ces deux strates a été mesurée séparément. La «densité» mesure le nombre de déjections au mètre carré. LAFONT (1953), a très justement mis en garde contre cette mesure qui, dans des conditions identiques de relevés, est cependant indicative de la

Tableau 4

Teneur en eau des sables à Arénicoles (Arcachon août 1965). Comparaison avec les sols d'herbiers immédiatement voisins

\begin{tabular}{|lcccccr|}
\hline Stations & La Hume & Cassy & Taussat & Andernos & Lège & $\begin{array}{c}\text { Sud de } \\
\text { l'tle }\end{array}$ \\
\hline Strate superficielle & $27^{0 \%}$ & 29 & 41 & 28 & 31 & 25 \\
Strate profonde & 24 & 20 & 36 & 24 & 20 & 21 \\
«Densité des arénicoles» & 143 & 75 & 61 & 78 & 54 & 137 \\
Herbiers voisins surface & 38 & 134 & 116 & 215 & 245 & 95 \\
Herbiers voisins fond & 30 & 38 & 41 & 33 & 128 & 32 \\
\hline
\end{tabular}


densité réelle des animaux. Les résultats sont rassemblés dans le Tableau 4. A chacune des six stations prospectées correspondent vingt échantillons, prélevés en quinconce à 1 mètre de distance les uns des autres, dont seules les moyennes figurent sur le tableau.

\section{Porosité}

Ruller (1959), signale que l'aire de répartition d'Arenicola marina dans l'Aber de Roscoff «correspond à la zone où l'on trouve 0 à $10 \%$ d'air et donc environ $20 \%$ d'eau d'imbibition du sable». La porosité totale des sables à arénicoles d'Arcachon est, sur l'Ile aux Oiseaux, de 34 à $37 \%$ en haut de plage (au pied des premières spartines), contre 21 à $28 \%$ en bas de plage (au contact des faciès de Leiochone clypeata). A la Hume, j'ai mesuré 23 à $31 \%$ en bas de plage (au contact de l'herbier), contre 34 à $42 \%$ en haut de plage (au contact des spartines). A cette porosité totale correspond un volume en air nul en bas de plage, mais qui peut atteindre 7 à 10\% en haut de plage. Ces chiffres sont du même ordre que ceux donnés par Amoureux (1966). Mais la porosité totale augmente brutalement lors de l'arrivée du flot, ce dont témoigne le fait que le pied s'enfonce davantage dans le sable recouvert d'une faible épaisseur d'eau, que dans le sable sec. J'ai mesuré la porosité du sédiment peu après le flot, sous $20 \mathrm{~cm}$ d'eau environ, au moyen d'un cube creux, enfoncé verticalement dans le sable, et dont les deux faces horizontales mobiles étaient refermées après l'enfoncement du cube. Les mesures sont, dans ces conditions, difficiles et très peu précises. Elles montraient cependant que, dans la demi-heure qui suit l'inondation, la porosité augmente rapidement, atteignant alors 50 à $60 \%$. Par ce mécanisme, la strate superficielle «respire» au rythme de la marée et s'oxygène. WeLls (1957), a montré l'importance de cette strate oxygénée, riche en microfaune et microflore, qui est la source alimentaire des arénicoles.

\section{Salinité des eaux interstitielles}

Le graphique Figure 4 montre l'évolution de la salinité des eaux d'imbibition des sables à arénicoles de l'lle aux Oiseaux au cours de l'année 1962. J'ai figuré simultanément le graphique de l'évolution de la salinité des eaux reouvrantes (assimilées ici à Eyrac). Il y a un parallélisme étroit entre l'évolution des eaux interstitielles et celle des eaux recouvrantes, qui contraste notamment avec ce que nous avons noté dans les herbiers. Cette remarque témoigne de la bonne perméabilité des sables à arénicoles. Ce faciès se développe cependant dans des zones où la salinité des eaux recouvrantes est très variable.

Au sud de la dune de Lillan, dans un petit estey qui draine à marée basse des eaux presque douce, j'ai mesuré en mars 1962 les salinités suivantes: (1) en aval, les arénicoles sont denses et cohabitent avec Nereis diversicolor; la salinité des eaux interstitielles est de $18,8 \%$; (2) en remontant le cours de 100 mètres en amont, les arénicoles quittent l'axe de l'estey, traversé par une eau totalement douce mais encore occupé par les Nereis, et se localisent sur les berges humides, imprégnées par une eau dont la salinité est de 10,4\% (3) environ 150 mètres plus en amont, les arénicoles 
sont devenues très rares, un individu tous les 2 ou 3 mètres carrés; la salinité est de $9,6 \%$.

Sur les rives du courant de Lège, les arénicoles sont localisées à l'embouchure de la rivière dont le lit est creusé entre les herbiers de zostères. Les arénicoles disparaissent très vite en amont par suite de la dessalure, la rivière drainant durant plusieurs heures,

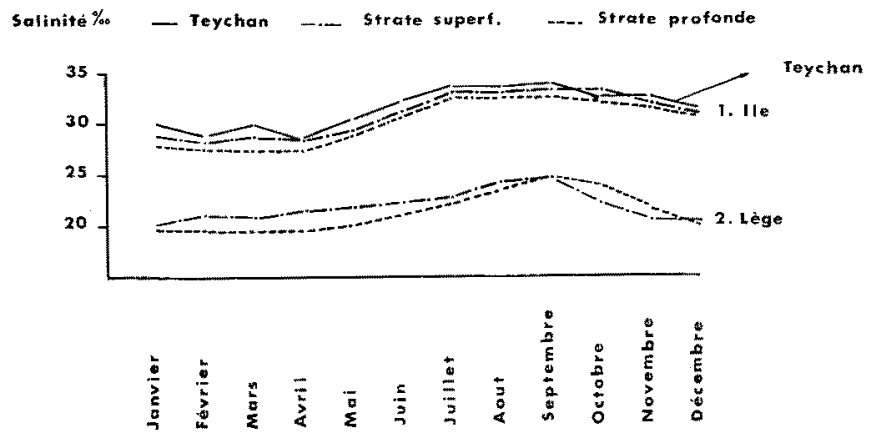

Fig. 4: Graphique de l'évolution annuelle de salinité des eaux interstitielles des sables à arénicoles. Lège et Ile aux Oiseaux. Comparaison avec le chenal du Teychan à Eyrac



Fig. 5: Le courant de Lège. Le débouché d'un estey venant du schorre, s'ouv rant dans la rivière. La photographie est prise au second point $A$. de la Figure 14. Dans le lit mêtme de la rivière, il n'y a plus d'arénicoles. Mais au débouché de l'estey, qui draine exclusivement les eaux salées qui recouvrent le schorre à marée haute, se développent de petits deltas où réapparaissent quelques arénicoles (au premier plan) 
à marée basse, une eau douce. Mais les arénicoles réapparaissent localement, aux débouchés des petits esteys qui se jettent dans le courant, après avoir drainé les eaux salées du schorre. Le 15 septembre 1964, alors que la rivière drainait une eau totalement douce à marée basse, les trois esteys explorés drainaient des eaux dont les salinités étaient respectivement de $13,2 \%, 11,4 \%$ et $21,9 \%$ (Fig. 5).

\section{$p H$}

Mesures in situ avec pH mètre Metrohm sur piles (juillet 1963). Nord de l'lle aux Oiseaux: de 7,25 à 7,65; Sud de l'Hle aux Oiseaux: de 7,45 à 7,70; La Hume: de 7,30 à 7,55; Lège-Arès: de 7,10 à 7,50 .

\section{Répartition bypsométrique}

Les sables à arénicoles couvrent de vastes étendues entre les herbiers de Zostères et les premières spartines de la haute slikke. Les arénicoles peuvent, en outre, coloniser le lit des esteys, atteignant alors des niveaux élevés. Excluant ces cas extrêmes, dont l'interprétation relève de conditions locales particulières, je préciserai la position hypsométrique de ces sables à partir d'exemples pris à la Hume, sur l'lle aux Oiseaux, et dans la région de Lège, où ils se présentent sous leur aspect le plus typique.

La Hume: Le long d'une radiale N.S., passant par le point géodésique GujanMestras $\mathrm{V}$, les arénicoles occupent, sur une profondeur de 300 mètres, distance qui sépare en cet endroit les spartines des zostères, l'espace de grève compris entre les cotes $+2,92 \mathrm{~m}$ et $+2,42 \mathrm{~m}$.

Ile aux Oiseaux: plage sud, radiale N.S. passant par les Tchanquées. Les arénicoles occupent, sur une profondeur de 250 mètres, l'espace de grève compris entre les cotes $+2,98 \mathrm{~m}$ et $+1,97 \mathrm{~m}$; plage est, radiale E.W. passant par le puit artésien. Les arénicoles occupent, sur une profondeur d'environ 300 mètres, l'espace de grève compris entre les cotes $+3,18 \mathrm{~m}$ et $+2,56 \mathrm{~m}$. Elles colonisent en outre l'herbier jusqu'à la cote $+2,25 \mathrm{~m}$.

Lège-Arès: Mesures discontinues entre $+2,40 \mathrm{~m}$ et $+3,00 \mathrm{~m}$.

Ces chiffres font ressortir que le faciès des sables à arénicoles se distribue, en définitive, autour de la Baie d'Arcachon, dans une strate d'assez faible amplitude.

\section{Les vases à Nereis diversicolor}

Nous prendrons le terme de «vase» dans le sens défini par Debyser (1961) «le terme de vase caractérise un état et non la nature pétrographique du sédiment». Les vases à Nereis diversicolor d'Arcachon sont, en fait, le plus souvent des sables vaseux et réduits, dans lesquels la fraction fine, inférieure à $50 \mu$, est pondéralement faible. Outre les vases qu'il nomme, Nereis diversicolor est susceptible de coloniser de nombreux autres territoires de manière plus ou moins sporadique. Nous considérons done les 
vases à Nereis diversicolor comme un faciès, au sens biocénotique strict, c'est à dire les aires où cette espèce constitue des peuplements denses et dans lesquelles la faune d'accompagnement est réduite.

\section{Teneur en eau}

Dix stations repérées sur la carte Figure 2, et notées ND 1 à ND 10, ont été explorées. Chaque station comprend vingt échantillons, prélevés en quinconce à 1 mètre de distance; les chiffres rapportés sur le Tableau 5 représentent la moyenne des mesures relatives aux vingt échantillons de chaque station. Dans toutes les stations ND 1 à ND 10, la densité de Nereis diversicolor dépassait 500 individus au mètre carré. La teneur en eau est élevée, homogène dans toute l'épaisseur du sédiment colonisé (aussi n'ai je pas discerné à son sujet diverses strates), comprise généralement entre 60 et $100 \%$, sauf pour ND 5 Lanton et ND 6 Comprian. Dans ces deux cas, il s'agissait non pas d'un véritable sédiment, mais d'un feutrage organique, dont la perte de poids par calcination au four était de $24 \%$ pour ND 5 et $31 \%$ pour ND 6.

Tableau 5

Vases à Nereis diversicolor. Stations N.D. 1 à N.D. 10

(Tableau des teneurs en eau et salinités des eaux interstitielles)

\begin{tabular}{|c|c|c|c|c|c|}
\hline \multirow{2}{*}{ Stations } & \multirow{2}{*}{$\begin{array}{c}\text { Teneur } \\
\text { en eau } \\
(0 / 0) \\
\text { Strate } \\
0-15\end{array}$} & \multicolumn{4}{|c|}{ Salinités (\%0) } \\
\hline & & Flaques & $\begin{array}{c}\text { Strate } \\
0-5\end{array}$ & $\begin{array}{c}\text { Strate } \\
5-10\end{array}$ & $\begin{array}{l}\text { Strate } \\
10-15\end{array}$ \\
\hline $\begin{array}{l}\text { N.D, } 1 \text { Jean de Boye } \\
16 \text { février } 1962\end{array}$ & 87 & 4,1 & 12,3 & 10,4 & 28,4 \\
\hline $\begin{array}{l}\text { N.D. } 2 \text { Hourquet } \\
15 \text { Mars } 1962\end{array}$ & 96 & 13,2 & 39,4 & 28,5 & 34,5 \\
\hline $\begin{array}{l}\text { N.D. } 3 \text { Dune de Lillan } \\
20 \text { juin } 1961\end{array}$ & 69 & 6,0 & & & 26,0 \\
\hline $\begin{array}{l}\text { N.D. } 4 \text { Lanton } \\
7 \text { septembre } 1961\end{array}$ & 89 & 15,6 & 28,7 & 23,6 & 40,7 \\
\hline $\begin{array}{l}\text { N.D. } 5 \text { Lanton } \\
7 \text { septembre } 1961\end{array}$ & 316 & 16,0 & & & 43,0 \\
\hline $\begin{array}{l}\text { N.D. } 6 \text { Comprian } \\
4 \text { janvier } 1961\end{array}$ & 710 & 2,0 & & & 8,0 \\
\hline $\begin{array}{l}\text { N.D. } 7 \text { Le Teich } \\
29 \text { novembre } 1961\end{array}$ & 61 & 1,8 & 2,0 & 6,3 & 23,9 \\
\hline $\begin{array}{l}\text { N.D. } 8 \text { La Teste } \\
7 \text { septembre } 1961\end{array}$ & 84 & 31,3 & 31,0 & 30,5 & 38,3 \\
\hline $\begin{array}{l}\text { N.D. 9 La Teste } \\
29 \text { novembre } 1961\end{array}$ & 88 & 22,7 & 22,0 & 18,3 & 22,4 \\
\hline $\begin{array}{l}\text { N.D. } 10 \text { Ile aux Oiseaux } \\
8 \text { février } 1962\end{array}$ & 71 & 26,4 & & & 28,4 \\
\hline
\end{tabular}




\section{Salinité des eaux interstitielles}

Pour l'analyse des salinités des eaux interstitielles (Tab. 5) j'ai distingué 3 strates de sédiment $(0-5 \mathrm{~cm},-5-10 \mathrm{~cm},-10-15 \mathrm{~cm})$ dont les salinités ont été comparées entre elles et à celles des flaques. La salinité est généralement plus élevée dans la strate profonde que dans la strate superficielle, contrairement aux sols d'herbiers, où c'est l'inverse, et aux sables à arénicoles, où les deux strates sont à ce point de vue identiques.

\section{$p H$}

Mesures in situ avec $\mathrm{pH}$ mètre Métrohm sur piles (juillet 1963). Le Teich: de 7,0 à 7,75 ; Arès: de 6,7 à 7,75 ; Claouey: de 6,8 à 7,4 . Les vases à Nereis sont souvent légèrement acides. Les zones de meilleure densité ont habituellement un $\mathrm{pH}$ inférieur à 7,5 .

\section{Autres habitats de Nereis diversicolor}

Outre son habitat préférentiel, celui des vases nues de la slikke, Nereis diversicolor occupe encore des biotopes variés.

(1) Les bancs de sable propre, mais très dessalé, qui jalonnent le lit des estuaires. Par exemple la station N.D. 11 se situe dans l'estuaire de la Leyre, entre les réservoirs de Bayonne et l'ile de Malprat. Nereis diversicolor, dont la densité ne dépasse pas une

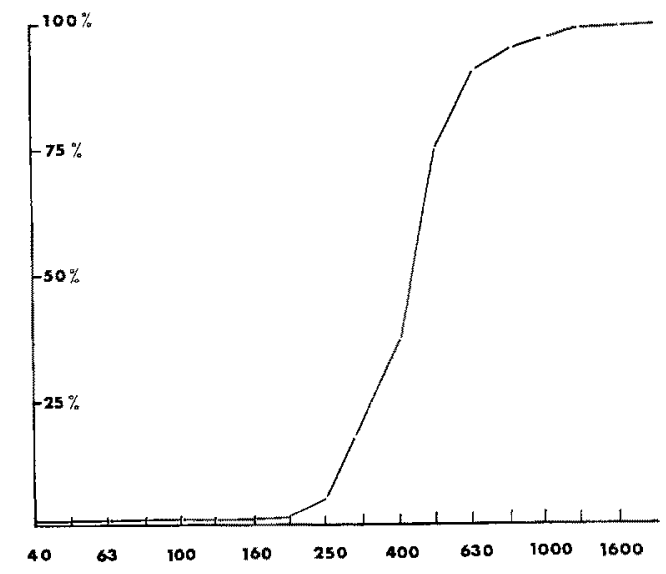

Fig. 6: Granulométrie station N.D. 11 La Leyre

cinquantaine d'individus au mètre carré, est le seul représentant de l'endofaune; le sédiment est constitué par un sable assez grossier, l'eau interstitielle a habituellement une salinité faible ou nulle mais qui augmente quelque peu après des marées de fort coefficient ( $S=11,5 \%$ le 5 septembre 1963 à basse mer, après une marée du coeffi- 
cient 104). Plus en aval, le fleuve s'étale soit sur des vases à Nereis diversicolor typiques, soit sur des sables à arénicoles où cohabitent en fait arénicoles et Nereis.

(2) Les prairies hygrophiles saumâtres du Juncetum maritimi de la région de Lège. Nereis diversicolor s'étend au sud de la dune de Lillan, depuis les vases nues et les herbiers de la slikke, jusqu'à un niveau élevé, puisqu'il atteint la cote $+3,73 \mathrm{~m}$. Son habitat se situe alors entre les touffes de Juncus maritimus, en position médiolittorale, dans des faciès référables à la haute slikke, voire au schorre. Ces prairies présentent en effet une originalité marquée, dûe à leur position élevée dans l'estran et, simultanément, à la présence à proximité de la surface, d'une nappe d'eau douce d'origine continentale, plus ou moins mêlée cependant d'apports marins. La forte teneur en eau des sols de ces prairies, liée à cette nappe, permet par conséquent le maintien d'une endofaune infra ou médiolittorale, référable à la slikke ou à la haute slikke, mais dans laquelle sont sélectionnées les espèces les plus euryhalines, c'est à dire essentiellement Nereis diversicolor, qui y pullule. Les rares inondations par la marée, liées à la position hypsométrique, permettent simultanément l'installation d'une épifaune référable au schorre, (Orchestia gammarella, divers insectes, notamment des carabiques et des homoptères, diverses araignées etc.).

(3) Les vases nues à strate profonde dessalée, que nous étudierons à propos du faciès des vases à Scrobicularia plana. Nereis diversicolor y atteint une densité parfois aussi élevée que celle qu'il atteint dans les faciès qu'il nomme.

\section{Répartition bypsométrique des populations de Nereis diversicolor}

Diverses aires où Nereis diversicolor constitue des populations denses ont été nivelées autour de la baie d'Arcachon.

La Teste: Les Nereis diversicolor les plus bas apparaissent à la cote $+2,38 \mathrm{~m}$, associés à Scrobicularia plana, dans le lit de l'estey qui traverse les marécages dits prés-salés. Non loin de là ils abondent à la cote $+2,69 \mathrm{~m}$, sur des vases molles typiques puis, à 2,95 $\mathrm{m}$ de nouveau associés aux scrobiculaires. Ils culminent enfin sur le contrefort de la route, au voisinage du pont qui franchit la «craste douce», à la cote $+3,39 \mathrm{~m}$, soit très peu au-dessous des plus hautes spartines de cette région $(+3,44 \mathrm{~m})$. Au Lapin Blanc, les Nereis ne descendent pas au dessous de $+2,84 \mathrm{~m}$, limités aux vases molles qui bordent la frange supérieure de l'herbier de zostères.

Gujan. A la sortie du port de Larros, les vases à Nereis débutent à $+2,84 \mathrm{~m}$. Elles se prolongent jusque vers $+3,50 \mathrm{~m}$, parmi les spartines.

Metras. Les vases à Nereis font irrégulièrement suite aux herbiers de zostères audelà de $+2,80 \mathrm{~m}$. Elles se prolongent jusqu'à $+3,58 \mathrm{~m}$, souvent au milieu des spartines. Beaucoup plus haut dans le schorre, j’ai noté la présence de peuplements de Nereis dans le fond d'un ancien lac de tonne (lac amènagé pour la chasse aux canards), envahi par des vases nues, et situé à la cote $+4,28 \mathrm{~m}$.

Le Teich. Dans la petite baie située au nord des réservoirs de Cantarane, les vases à Nereis débutent vers $+2,50 \mathrm{~m}$ et culminent au fond de la baie à la cote $+3,50 \mathrm{~m}$. A la faveur des dépressions du schorre, on rencontre encore des peuplements sur fond de vase à la cote $+3,80 \mathrm{~m}$. 
Certes. Le long de l'estey qui conduit au port de Certes, les vases à Nereis diversicolor s'étendent sur les rives depuis la cote $+2,25 \mathrm{~m}$ jusqu'à la cote $+2,68 \mathrm{~m}$. Au nord des réservoirs de Certes, dans la petite baie de Lanton, les Nereis occupent de vastes étendues de vases molles entre le sommet de l'herbier de zostère, soit $+2,95 \mathrm{~m}$, et la cote $+3,50 \mathrm{~m}$, où elles s'éteignent au milieu des spartines. Les polychètes se retrouvent encore à la cote $+3,80 \mathrm{~m}$ dans les cuvettes à fond de vase nue sur le schorre.

Lège. Dans la région de Lège, les sables vaseux à Nereis et Scrobicularia descendent au moins jusqu'à la cote $+2,69 \mathrm{~m}$. Lorsque l'on passe de la slikke nue à la haute slikke à végétation phanérogamique dressée, les scrobiculaires disparaissent, tandis que les Nereis persistent non seulement entre les pieds de spartines qui, dans cette région, culminent vers $+3,35 \mathrm{~m}$, mais encore parmi les Juncus qui croissent sur un sol spongieux très humide; le faciès Nereis + Juncus (accompagné par l'épifaune vagile du schorre) s'étend sur plusieurs hectares, et culmine vers $+3,73 \mathrm{~m}$, donc très nettement au dessus de la frange supérieure des spartines.

Ile aux Oiseaux. Les peuplements denses de Nereis diversicolor débutent à la cote $+2,39 \mathrm{~m}$, sur les sables vaseux où se rencontrent également des scrobiculaires. En bordure de l'estey dit des Gnathia, les Nereis peuplent des sables propres, en compagnie de Nepbthys hombergii et d'Arenicola marina; dans la haute slikke ils remontent jusqu'à la cote $+3,39 \mathrm{~m}$.

\section{Remarques}

(1) Les nivellements rapportés concernent des mesures faites durant l'été 1963.

(2) Les résultats relatifs à la répartition hypsométrique des peuplements de Nereis diversicolor sont résumés dans le tableau çi-dessous (Tab. 6).

Tableau 6

Résultats relatifs à la répartition hypsométrique des peuplements de Nereis diversicolor

\begin{tabular}{|lccc|}
\hline Localités & Frange inferieure $(\mathrm{m})$ & Frange superieure $(\mathrm{m})$ & Cuvettes $(\mathrm{m})$ \\
\hline La Teste & 2,38 & 3,39 & \\
Gujan & 2,84 & 3,50 & \\
Mestras & 2,80 & 3,58 & 4,28 \\
Le Teich & 2,50 & 3,50 & 3,80 \\
Certes & 2,25 & 2,68 & 3,80 \\
Baie de Lanton & 2,95 & 3,50 & \\
Lège & 2,69 & 3,73 & \\
Ile aux Oiseaux & 2,39 & 3,39 & \\
\hline
\end{tabular}

La frange inférieure oscille entre les cotes extrêmes de $+2,25 \mathrm{~m}$ (port de Certes) et de $+2,95 \mathrm{~m}$ (Lanton), soit avec une amplitude de $0,70 \mathrm{~m}$. La frange supérieure, cuvettes exclues, entre $+3,73 \mathrm{~m}$ (Lège) et $+2,68 \mathrm{~m}$ (Certes), soit avec une amplitude $\mathrm{de}+1,05 \mathrm{~m}$.

(3) Sur les plages de vase Nereis diversicolor débute sensiblement au niveau de la bordure supérieure des zostères, occupe l'espace de slikke nue qui sépare les zostères des spartines, mais culmine habituellement au delà de la limite inférieure de ces dernières. 
(4) Cette répartition suggère que l'habitat préférentiel de Nereis diversicolor se situe dans des sols riches en eau, qu'ils soient ou non fréquemment inondés par la marée. La grande euryhalinité de cette espèce lui permet, en particulier dans la région de Lège, de coloniser dans presque toute leur étendue les prairies saumâtres du Juncetum maritimi, imprégnées d'une eau dont la faible salinité limite l'extension des autres peuplements de la slikke ou de la haute slikke, notamment des spartines.

(5) Parmi toutes les espèces de l'endofaune des plages abritées, Nereis diversicolor est celle qui présente la tendance la plus accusée à coloniser des niveaux élevés, au milieu même du schorre, à la faveur de dépressions ou de suintements qui entretiennent l'humidité. Lorsque, en été, la partie superficielle du sol se dessèche, Nereis diversicolor s'enfonce plus profondément. JACQUET (1949) cite cette espèce comme constituant de la faune du schorre, dans le havre de Regnéville. Je pense qu'il faut plutôt considérer les peuplements élevés de Nereis diversicolor, comme des enclaves de haute slikke dans le schorre.

\section{Les vases à Scrobicularia plana}

Le lamellibranche endogé Scrobicularia plana occupe des aires de dimensions variables dont le caractère saumâtre a été longuement discuté (MoNoD 1928, Fischer 1928, Spooner \& Moore 1940). Il est généralement considéré par les géologues comme spécifique des vases saumâtres, notamment avec les Myies (Bourcart \& Francrs-Boeur 1942). Les conditions écologiques de son habitat ont été bien décrites à Roscoff par Rullier (1959) et surtout Guerin (1961). Peres \& Picard (1964) classent les scrobiculaires comme caractéristiques de la biocénose lagunaire euryhaline et eurytherme. Le caractère largement euryhalin de Scrobicularia plana, a été étudié du point de vue du mécanisme de la régulation osmotique par FreEMAN \& Rigler (1957); il y a réalisation d'un équilibre osmotique avec le milieu extérieur; en cas de variation brusque de salinité, l'animal referme ses valves de manière à pouvoir réaliser progressivement son équilibre osmotique.

\section{Les vases à Scrobicularia plana de la régíon d'Arcachon}

Scrobicularia plana est largement répartie d'une part autour de l'tle aux Oiseaux, d'autre part au Nord Est d'une ligne La Teste-Claouey. Les scrobiculaires sont localisées principalement d'une part dans le cours moyen des grands esteys (Ile aux Oiseaux, littoral sud entre la Hume et le Teich), d'autre part dans les vases de la Slikke qui s'étendent au pied des dunes de la région de Lège et Arès (Dune de Lillan ou l'Illon).

Les caractéristiques des sols où stationnent les scrobiculaires ont été étudiées dans une dizaine de stations au cours des étés 1962 et 1963. Pour chaque station 5 à 6 gîtes individuels étaient explorés de la manière suivante: (1) un manchon cylindrique en aluminium de $12 \mathrm{~cm}$ de diamètre, centré sur la trace étoilée que dessine lanimal en surface, est enfoncé verticalement d'une dizaine de centimètres; on récolte alors la strate superficielle du sédiment. (2) le manchon est ensuite enfoncé jusqu'au niveau 
de la loge où est installé le mollusque et on prélève le sédiment autour de cette loge; (3) enfin on approfondit verticalement le trou, tout en enfonçant le manchon, jusqu'à $60 \mathrm{~cm}$ environ; sur une cinquantaine de gîtes ainsi explorés, j’ai noté 46 fois que l’on arrivait à un niveau de résurgence, où l'eau sourd très rapidement jusqu'à envahir en quelques secondes toute la cavité ainsi creusée, alors qu'au dessus de ce niveau, les suintements sont très faibles. Le Tableau 7 indique les caractéristiques de quelques gîtes choisis à titre d'exemple.

\section{Profondeur de la loge}

Elle est très variable d'une station à l'autre (de $10 \mathrm{~cm}$ en moyenne dans la slikke de Lillan, à $25 \mathrm{~cm}$ dans une station de l'lle aux Oiseaux), mais assez constante dans une même station.

\section{Température du sédiment}

A une même époque de l'année (prospections en aoùt), et dans une même station, la température du sédiment au niveau de la loge, et plus encore au niveau de résurgence, est à peu près constante et indépendante de la température de la strate superficielle; ainsi en août 1963, la température de la strate de résurgence était de $21^{\circ} \mathrm{C}$ à $22^{\circ} \mathrm{C}$, alors que dans la strate superficielle elle variait de $10^{\circ} \mathrm{C}$ à $28^{\circ} \mathrm{C}$.

\section{Teneur en eau}

La teneur en eau de la strate superficielle est toujours élevèe et plus élevée que celle des couches sous-jacentes. Le sédiment, aussi bien en surface qu'au fond, est toujours intégralement saturé d'eau mais, sa texture étant plus fine en surface qu'au fond (voir granulométrie), les couches superficielles fixent davantage d'eau que les couches profondes. La strate superficielle inclut en outre une certaine épaisseur de vase thixotropique très riche en eau.

\section{Salinité des eaux interstitielles}

L'eau interstitielle a été extraite mécaniquement au laboratoire, en pressant le sédiment entre les mâchoires d'un presse fruits; l'eau de résurgence est directement. recueillie sur place. La salinité de l'eau interstitielle de la strate superficielle est presque toujours plus élevée que celle de la strate profonde; toutefois il arrive que ce soit linverse (station S. p. 5) mais celà est rare ( 6 cas sur les 50 gîtes explorés). La dessalure de la strate profonde mérite d'autant plus d'être signalée que, nous l'avons vu à propos des vases à Nereis diversicolor, en l'absence d'apports souterrains, les vases de la slikke ont tendance à retenir en profondeur une eau plus salée que l'eau superficielle. 


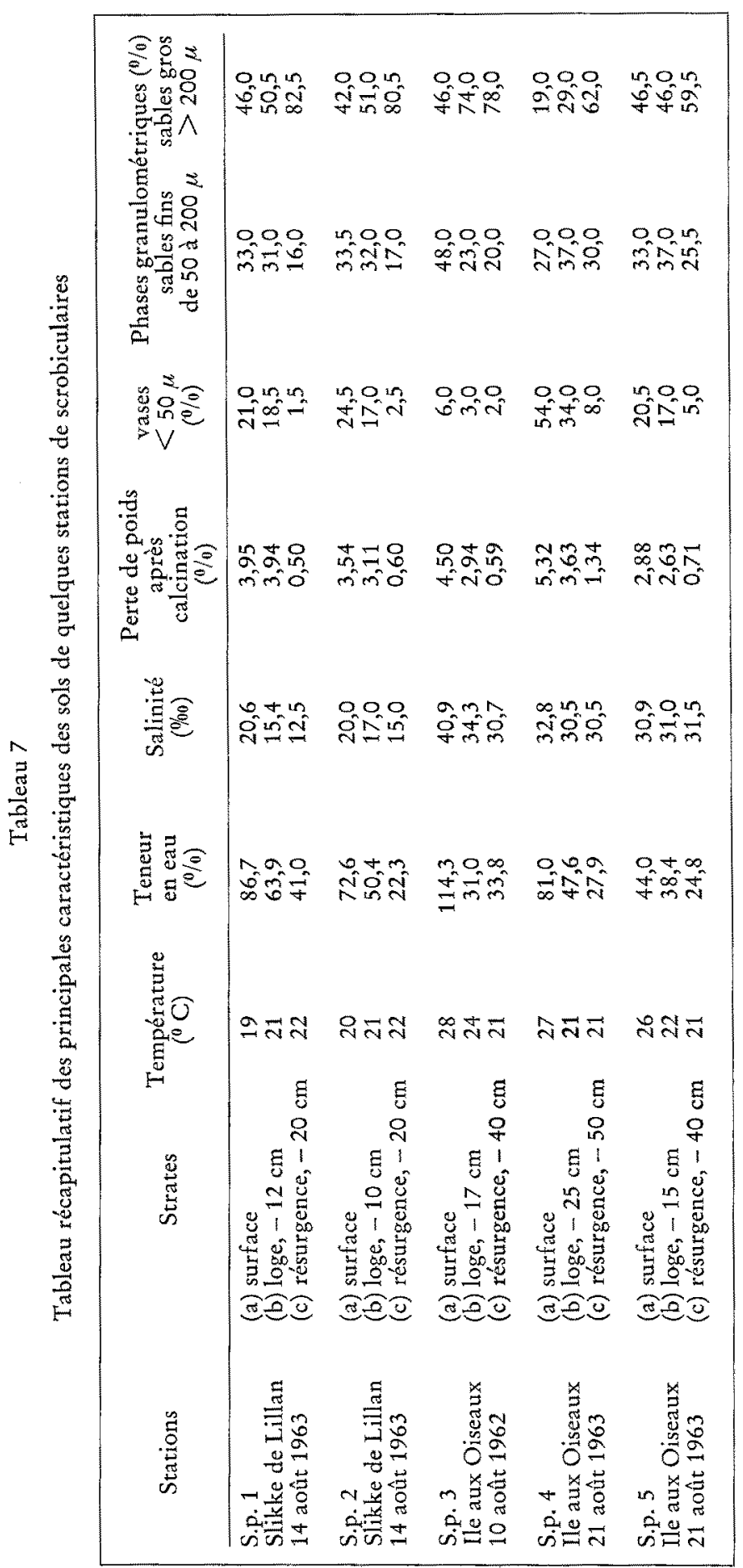


GUERIN (1961) signale que la teneur en oxygène dissous des eaux interstitielles des biotopes à scrobiculaires de la région de Roscoff est variable, mais parfois élevée, surtout dans le faciès des tâches. Autour de ces biotopes, la teneur en oxygène des eaux qui imprégnent les vases est au contraire faible ou nulle. GuErin ne donne pas d'indications sur la manière dont il a recueilli l'eau interstitielle. J'ai mesuré dans les stations explorées la teneur en oxygène dissous des eaux superficielles qui recouvrent le sédiment à marée basse d'une part, et des eaux profondes qui jaillissent au niveau de la nappe de résurgence d'autre part. La nappe superficielle n'est jamais complètemont réduite et peut même avoir une teneur en oxygène élevée (de 6 à $10 \mathrm{mg} /$ litre, en août 1962, à Baste Vieille); cette teneur toutefois ne diffère pas sensiblement de celle que l'on peut mesurer sur les flaques qui recouvrent les vases à Nereis diversicolor. Il est possible qu'un plus grand nombre de mesures fasse apparaître statistiquement des différences significatives. La teneur en oxygène dissous de la nappe qui sourd au niveau de résurgence est difficile à mesurer, par suite de l'apport de particules fines réductrices qui polluent les eaux. Cependant, chaque fois que l'eau qui sourd est limpide, on note une teneur en oxygène variable, rarement nulle, parfois assez élevée; les valeurs observées vont de 0,5 à $2,5 \mathrm{mg} /$ litre pour Lillan, en août 1962, et de 0,5 à $4 \mathrm{mg} /$ litre sur l'Ile aux Oiseaux à la même ćpoque ${ }^{2}$. En l'absence de nappe de résurgence dans les vases à Nereis diversicolor, il est difficile d'établir des comparaisons chiffrées. Toutefois, on note que les vases à Nereis diversicolor sont toujours réduites et de couleur noire, aussi bien en surface qu'au fond, alors que dans les vases à scrobiculaires le sédiment est beige clair en surface, généralement noir et réduit au niveau de la loge, gris et lègèrement oxydé au niveau de la nappe de résurgence. Guerin (1961) signale même que dans certains cas le sable des biotopes à scrobiculaires reste clair dans toute son épaisseur.

\section{Teneur en matière organique}

La perte de poids, exprimée en pourcentage par rapport au poids sec du sédiment, par calcination au four après déchloruration et décalcification, montre que la teneur en matière organique décroît de la surface au fond.

$$
p H
$$

Comme pour les vases à Nereis diversicolor, le $\mathrm{pH}$ des eaux interstitielles des biotopes à Scrobicularia plana est nettement moins alcalin que le $\mathrm{pH}$ de l'eau recouvrante, parfois même nettement acide. Sur l'Ile aux Oiseaux, le pH de l'eau de résur-

2 Ces chiffres méritent d'autant plus d'être retenus que divers auteurs ont, récemment encore, souligné (voir Amoureux 1963) la réduction des eaux interstitielles, particulièrement dans les plages abritées. 
gence se situe, pour 40 mesures en juillet-Août 1963, entre 6,8 et 7,8. A Lillan, le pH de l'eau de résurgence se situe, pour 30 mesures à la même époque, entre 6,5 et 8,1 ; en outre, dans deux gîtes, le $\mathrm{pH}$ atteignait exceptionnellement 6,0 .

\section{Granulométrie}

Les courbes de la Figure 7 (courbes cumulatives, à partir des fins, en coordonnées semi-logarithmiques, tamisages sur tamis de la série A.F.N.O.R.) montrent la texture des différentes strates du sédiment. Afin de faciliter les comparaisons j'ai indiqué sur le Tableau 7 le pourcentage des phases granulométriques retenues par GUERIN (1961). La fraction fine, inférieure à $50 \mu$, est assez élevée dans la strate superficielle, alors qu'elle est toujours très faible au niveau de la strate de résurgence; la différence est d'ailleurs suffisante pour être directement appréciable sur place, à l'oeil et au toucher. Les couches superficielles sont donc colmatées et peu perméables, alors que la couche profonde permet la libre circulation de l'eau. Au niveau de la loge creusée par l'animal, la structure du sédiment est généralement du type de la strate superficielle (par exemple station S. p. 1 ou S. p. 5) plus rarement du type de la strate de résurgence (S. p. 3).



Fig. 7: Courbes granulométriques cumulatives des différentes strates des sols à scrobiculaires

\section{Répartition bypsométrique}

Les aires référables au faciès de vases à scrobiculaires n'ont pu être nivelées avec la même continuité que celles des faciès précédents; les chiffres ci-dessous sont donnés à titre indicatif:

La Teste: base d'un peuplement: $+2,38 \mathrm{~m}$; sommet du même peuplement: $2,99 \mathrm{~m}$. La Hume: base $+2,50 \mathrm{~m}$; sommet $+2,96 \mathrm{~m}$. 
Lège: base $+2,69 \mathrm{~m}$; sommet $+3,27 \mathrm{~m}$.

Ile aux Oiseaux: base $+2,53 \mathrm{~m}$; sommet $+3,11 \mathrm{~m}$.

Dans l'ensemble, le faciès des vases à scrobiculaires se distribue donc dans des niveaux correspondant bien à la slikke, et culmine sensiblement au dessous du sommet des peuplements de Nereis diversicolor.

\section{Conclusions sur la slikke}

Les sols référables à la slikke ont généralement une haute teneur en eau libre. Cette haute teneur tient d'une part à la position de la slikke dans l'estran, d'autre part à la présence d'une couche de vase superficielle thixotropique. Du point de vue hypsométrique, tous les faciès référables à la slikke culminent habituellement peu audessus du niveau atteint par toutes les marées. Quant à la couche de vase thixotropique, elle constitue, suivant l'expression de Bourcart \& Francis Boevf (1942) un «liquide rigide», formant un réseau enfermant dans ses mailles la phase liquide. Elle joue un rôle écologique fondamental; en effet, la rigidité de cette vase empêche qu'elle ne s'écoule avec le jusant, comme le ferait une nappe d'eau; en même temps la facilité avec laquelle l'équilibre thixotropique est rompu, permet à la petite faune de se mouvoir dans cette couche superficielle, presque aussi facilement que dans l'eau; par exemple de jeunes flets peuvent y nager.

Les mécanismes des échanges entre l'eau recouvrante et les eaux interstitielles, sont variables suivant chaque faciès. Ainsi, dans les sables à arénicoles, peu colmatés, les échanges par perméabilité sont rapides, l'eau interstitielle est constamment remaniée par l'eau recouvrante, avec laquelle elle reste en équilibre (Fig. 4). Dans les vases à Nereis au contraire, et même dans les herbiers, interviennent davantage les échanges par densité; les eaux recouvrantes pénètrent le sédiment losqu'elles sont plus denses que les eaux interstitielles; il y a une nette tendance à la stratification des eaux interstitielles, en fonction de la densité. Callame (1960) envisage un troisième type d'échange, par diffusion moléculaire; un tel mécanisme est trop lent pour, semble-t-il, intervenir régulièrement sur les slikkes ouvertes mais son rôle est à envisager dans les flaques, notamment sur les herbiers en début d'automne (Fig. 3). Enfin, l'importance de la circulation et de la résurgence des eaux souterraines est clairement mise en évidence à propos des faciès à scrobiculaires.

En définitive, si la haute teneur en eau libre de la slikke est un facteur général qui conditionne le caractère infralittoral de l'ensemble de la faune endogée, en revanche l'hydrodynamisme interstitiel, très variable suivant les biotopes, est un facteur différentiel de chaque faciès.

\section{La faune de la slikke}

Les espèces des slikkes de la baie d'Arcachon sont rassemblées dans le Tableau 8. 
Tableau 8

Liste des espèces de la slikke

\begin{tabular}{|c|c|c|c|c|}
\hline Espèces & 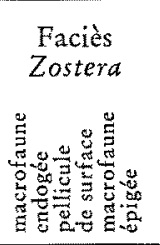 & 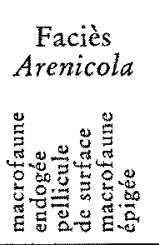 & 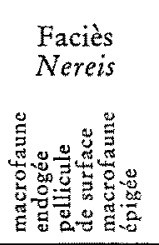 & 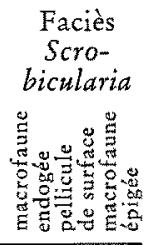 \\
\hline $\begin{array}{l}\text { Cnidaires Hexacoralliaires: } \\
\text { Cereus pedunculatus (PENNANT) } \\
\text { Ceriantbus membranaceus SPALL. } \\
\text { Diddumene luciae (VERRIL) } \\
\text { Edwarsia callimorpha (GossE) }\end{array}$ & $\begin{array}{l}+ \\
+ \\
+ \\
+\end{array}$ & & + & + \\
\hline $\begin{array}{l}\text { Annélides Polychètes: } \\
\text { Arabella iricolor (MONTAGU) } \\
\text { Diopatra neapolitana DELLE CHIAJE } \\
\text { Glycera convoluta KEFERSTEIN } \\
\text { Glycera unicornis SAVIGNY } \\
\text { Harmothoe lunulata (DELLE CHIAJE) } \\
\text { Lumbriconereis impatiens CLAP. } \\
\text { Lumbriconereis latreilli A. \& M. E. } \\
\text { Nephthys bombergii A. \& M. E. } \\
\text { Nereis diversicolor O. F. MÜLLER } \\
\text { Nereis irrorata (MALMGREN) } \\
\text { Pilargis verrucosa ST. JOSEPH } \\
\text { Phyllodoce mucosa OERSTED } \\
\text { Platynereis dumerilii (A. \& M. E.) } \\
\text { Ampharete grubei MALMGREN } \\
\text { Arenicola marina (LINNÉ) } \\
\text { Aricia foetida CLAPARĖDE } \\
\text { Audoninia tentaculata (MONTAGU) } \\
\text { Capitella capitata (FABRICIUS) } \\
\text { Heteromastus fliformis (CLAP.) } \\
\text { Leiochone clypeata ST. JOSEPH } \\
\text { Melinna palmata GRUBE } \\
\text { Owenia fusiformis DELLE CHIAJE } \\
\text { Polymnia nebulosa (MONTAGU) } \\
\text { Prionospio malmgreni CLAPARÈDE } \\
\text { Sabella pavonina SAVIGNY } \\
\text { Scoloplos armiger (O. F. MÜLLER) } \\
\text { Streblospio shrubsolii (BUCHANAN) }\end{array}$ & $\begin{array}{l}+ \\
+ \\
+ \\
+ \\
+ \\
+ \\
+ \\
+\end{array}$ & $\begin{array}{l}+ \\
+ \\
+ \\
+ \\
+ \\
+ \\
+ \\
+ \\
+ \\
+ \\
+ \\
+ \\
+ \\
+ \\
+ \\
+ \\
+ \\
+ \\
+ \\
+ \\
+ \\
+ \\
+ \\
+ \\
+ \\
+ \\
+\end{array}$ & + & + \\
\hline $\begin{array}{l}\text { Annélides Oligochètes: } \\
\text { Paranais littoralis (MÜLLER) } \\
\text { Peloscolex benedeni (UDEREM) } \\
\text { Tubifex costatus (CLAPARÈDE) }\end{array}$ & & & $\begin{array}{l}+ \\
+ \\
+\end{array}$ & \\
\hline $\begin{array}{l}\text { Mollusques Gastéropodes: } \\
\text { Bittium reticulatum (DA CosTA) } \\
\text { Gibbula cineraria (L.) } \\
\text { Gibbula magus (L.) } \\
\text { Gibbula umbilicalis (DA CosTA) } \\
\text { Hydrobia ulvae (PENNANT) } \\
\text { Littorina littorea (L.) } \\
\text { Littorina obtusata (L.) } \\
\text { Rissoa sp. } \\
\text { Acera bullata O. F. MüLLER }\end{array}$ & $\begin{array}{r}+ \\
+ \\
+ \\
+ \\
+ \\
+ \\
+ \\
+ \\
+\end{array}$ & + & ++ & ++ \\
\hline
\end{tabular}


Tableau 8 (suite)

\begin{tabular}{|c|c|c|c|c|}
\hline \multirow[t]{2}{*}{ Espèces } & $\begin{array}{l}\text { Faciès } \\
\text { Zostera }\end{array}$ & $\begin{array}{c}\text { Faciès } \\
\text { Arenicola }\end{array}$ & $\begin{array}{l}\text { Faciès } \\
\text { Nereis }\end{array}$ & $\begin{array}{c}\text { Faciès } \\
\text { Scro- } \\
\text { bicularia }\end{array}$ \\
\hline & 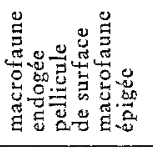 &  & 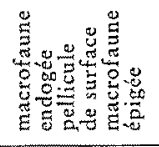 &  \\
\hline $\begin{array}{l}\text { Cylicbna cylindracea (PENNANT) } \\
\text { Haminea navicula (DA CosTA) } \\
\text { Retusa truncatula (BRUgutere) }\end{array}$ & $\begin{array}{l}++ \\
+t \\
++\end{array}$ & & & \\
\hline 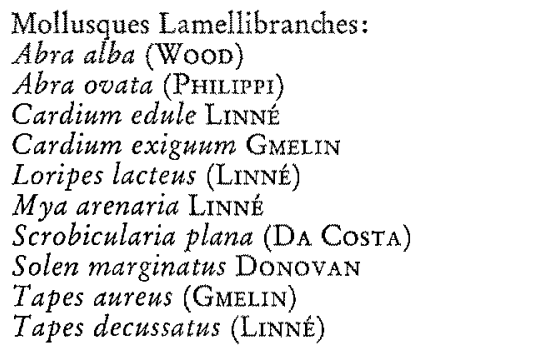 & $\begin{array}{l}+ \\
+ \\
+ \\
+ \\
+ \\
+ \\
+ \\
+ \\
+\end{array}$ & $\begin{array}{l}+ \\
+\end{array}$ & + & + \\
\hline $\begin{array}{l}\text { Crustacés Ostracodes: } \\
\text { Cyprideis torosa (JoNes) } \\
\text { Cytherois fischeri (SARS) } \\
\text { Leptocythere castanea (SARS) } \\
\text { Leptocythere fabaeformis (G. W. MÜLLER) } \\
\text { Leptocythere pellucida (BAIRD) } \\
\text { Loxoconcha elliptica BRADY } \\
\text { Paradoxostomaturabile (BAIRD) } \\
\text { Xestoleberis atrantia (BATRD) }\end{array}$ & $\begin{array}{l}+ \\
+ \\
+ \\
+ \\
+ \\
+ \\
+\end{array}$ & & $\begin{array}{l}+ \\
+ \\
+ \\
+\end{array}$ & $\begin{array}{l}+ \\
+ \\
+ \\
+ \\
+ \\
+\end{array}$ \\
\hline $\begin{array}{l}\text { Crustacés Mysidacés: } \\
\text { Neomysis integer (LEACH) } \\
\text { Paramysis nouveli LARAT }\end{array}$ & & + & + & + \\
\hline $\begin{array}{l}\text { Crustacés Tanaidacés: } \\
\text { Heterotanaïs oerstedi (KRöYER) } \\
\text { Leptocbelia dubia (KRÖYER) }\end{array}$ & + & & + & \\
\hline $\begin{array}{l}\text { Crustacés Isopodes: } \\
\text { Cyathura carinata (KRÖYER) } \\
\text { Eurydice pulcbra LEACH } \\
\text { Idotea viridis (SLABBER) } \\
\text { Sphaeroma rugicauda LEACH }\end{array}$ & $\begin{array}{l}++ \\
++ \\
++\end{array}$ & $+{ }^{+}$ & + & + \\
\hline $\begin{array}{l}\text { Crustacés Amphipodes: } \\
\text { Ampelisca brevicornis (A. COSTA) } \\
\text { Apberusa bispinosa (BATE) } \\
\text { Batbyporeia elegans WATKIN } \\
\text { Batbyporeia pilosa LnNDSRÖM } \\
\text { Corophinm insidiosum CRA WFORD } \\
\text { Corophitum sextoni CRAWFORD } \\
\text { Corophium volutator (PALLAS) } \\
\text { Dexamine spinosa (MONTAGU) } \\
\text { Erichthonius brasiliensis (DANA) } \\
\text { Erichthonius difformis H. M. E. } \\
\text { Hyale nilssoni (RATHEE) } \\
\text { Gammarus aequicauda MARTYNOV }\end{array}$ & $\begin{array}{l}+ \\
+ \\
+ \\
+ \\
+ \\
+ \\
+\end{array}$ & $\begin{array}{l}+ \\
+ \\
+\end{array}$ & & $\begin{array}{r}+ \\
++\end{array}$ \\
\hline
\end{tabular}


Tableau 8 (suite)

\begin{tabular}{|c|c|c|c|c|}
\hline \multirow[t]{2}{*}{ Espèces } & $\begin{array}{l}\text { Faciès } \\
\text { Zostera }\end{array}$ & $\begin{array}{l}\text { Faciès } \\
\text { Arenicola }\end{array}$ & $\begin{array}{l}\text { Faciès } \\
\text { Nereis }\end{array}$ & $\begin{array}{c}\text { Faciès } \\
\text { Scro- } \\
\text { bicularia }\end{array}$ \\
\hline & 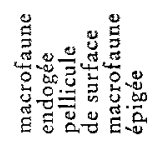 &  &  & 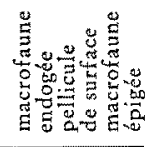 \\
\hline $\begin{array}{l}\text { Marinogammarus marinus (LEACH) } \\
\text { Melita palmata (MoNTAGU) } \\
\text { Microdentopus anomalus (RATHKE) } \\
\text { Microdeutopus gryllotalpa A. COSTA } \\
\text { Microdeutopus stationis DELLA VALLE }\end{array}$ & $\begin{array}{l}+ \\
+ \\
+ \\
+ \\
+\end{array}$ & & + & \\
\hline $\begin{array}{l}\text { Crustacés Decapodes: } \\
\text { Crangon crangon (L.) } \\
\text { Hippolyte sp. } \\
\text { Leander sp. } \\
\text { Palaemonetes varians (LEACH) } \\
\text { Upogebia pusilla (PETAGNA) } \\
\text { Clibanarius misanthropus (RIsso) } \\
\text { Carcinus maenas (L.) }\end{array}$ & $\begin{array}{l}+ \\
+ \\
+ \\
+ \\
+ \\
+ \\
+\end{array}$ & $\begin{array}{r}+ \\
+ \\
+\end{array}$ & + & + \\
\hline $\begin{array}{l}\text { Insectes Diptères (larves): } \\
\text { Ceratopogonidae } \\
\text { Chironomidae } \\
\text { Ceratinostoma ostiorum } \mathrm{H}_{\mathrm{AL}} . \\
\text { Coelopa frigida FALL. } \\
\text { Fucellia maritima HAL. }\end{array}$ & $\begin{array}{l}+ \\
+ \\
+ \\
+ \\
+\end{array}$ & & + & + \\
\hline $\begin{array}{l}\text { Echinodermes: } \\
\text { Labidoplax digitata (MONTAGU) } \\
\text { Leptosynapta galliennei (HERAPATH) } \\
\text { Paracentrotus lividus (LAMARCK) } \\
\text { Psammechinus miliaris (GMELIN) } \\
\text { Asterias rubens (L.) }\end{array}$ & + & + & & \\
\hline $\begin{array}{l}\text { Tuniciers Ascidies: } \\
\text { Molgula manhattensis DE KAY }\end{array}$ & + & & & \\
\hline $\begin{array}{l}\text { Vertébres Téléostéens: } \\
\text { Anguilla anguilla (L.) } \\
\text { Arnoglossus thori KYLE } \\
\text { Crenilabrus sp. }\end{array}$ & $\begin{array}{l}+ \\
+ \\
+\end{array}$ & + & & \\
\hline $\begin{array}{l}\text { Gobius microps KRÖYER } \\
\text { Gobius minutus PALLAS }\end{array}$ & + & + & + & + \\
\hline $\begin{array}{l}\text { Gobius niger L. } \\
\text { Mugil sp. }\end{array}$ & $\begin{array}{l}+ \\
+\end{array}$ & & & \\
\hline $\begin{array}{l}\text { Platichthys flesus (L.) } \\
\text { Solea solea (L.). }\end{array}$ & $\begin{array}{l}+ \\
+\end{array}$ & $\begin{array}{l}+ \\
+\end{array}$ & + & + \\
\hline Syngnathidae divers & + & & & \\
\hline
\end{tabular}

Remarques systématiques et écologiques

\section{Cnidaires Hexacoralliaires}

Cereus pedunculatus (Pennant); considéré par True Schlenz (1965), comme espèce caractéristique exclusive des herbiers méditerranéens de petites zostères; abondant à 
Arcachon certaines années (1962-1963) et sur certains herbiers (Nord de l'lle aux Oiseaux). En 1963, cette espèce était extrêmement dense dans les sables vaseux profonds, inférieurs au niveau de l'herbier, dans la lagune de Bernet. Spooner \& Moore (1940) ont déjà signalé la tendance de cette espèce à constituer des populations très denses ( 770 par mètre carré) sur les vases molles.

Ceriantbus membranaceus SPALL; localisé dans la partie inférieure des herbiers sablovaseux, s'étend aussi dans l'herbier de Zostera marina. Autrefois abondant dans la baie d'Arcachon, déjà signalé en régression par LUBET (1956), actuellement très rare.

Diadumene luciae (VERRIL), (= Sagartia luciae); petite espèce minuticole, qui appartient également à la haute slikke; rare dans l'herbier si ce n'est à l'est de Claouey, où elle pullule sur les vases molles où croissent les plus hautes zostères; abondante également dans les vases à Nereis diversicolor.

Edwarsia callimorpha (Gosse); recolté régulièrement sur herbiers des Hosses (Ile aux Oiseaux); accidentel ailleurs.

\section{Annélides Polychètes}

Malgré son grand intérêt, je ne m'attarderai pas sur ce groupe, qui a fait l'objet d'une publication de Borsseau (1962) et sur lequel C. Cazaux prépare actuellement un important travail d'ensemble.

Harmothoe lunulata (Delle Chiaje); Fauvel (1927) \& Borsseau (1962), signalent à Arcachon la variété synaptae, abondante sur les plages à synaptes (Eyrac, CapFerret); sur les plages à arénicoles de l'Ile aux Oiseaux et de la Hume, j'ai récolté une variété beaucoup plus sombre.

Nereis diversicolor O. F. MüzLER; présent dans tous les faciès de la slikke, pullule dans les vases qu'il nomme, ainsi que dans les vases à scrobiculaires. Beaucoup plus rare ailleurs. Dans l'estuaire des ruisseaux, il prend, vers l'amont, la suite des arénicoles, lorsque la salinité s'abaisse au dessous de 10\%. Dans un herbier saumâtre de la Sableyre, il constitue l'unique élément de l'endofaune, l'épifaune étant représentée par Idotea viridis. Dans les plages abritées sablo-vaseuses, c'est le seul polychète susceptible de coloniser les faciès médiolittoraux de la haute slikke.

Capitella capitata (Fabricius); remonte très loin dans le fond de la baie, s'étend notamment dans une bonne partie des vases molles à Nereis diversicolor (Claouey, le Teich); accidentel dans l'herbier.

Heteromastus filiformis (CLAP.); très abondant dans les sables vaseux avec ou sans herbier.

Leiochone clypeata ST. JosEpH; extrêmement dense dans les sables nus, légèrement vaseux, intercalés topographiquement entre l'herbier et la haute slikke. Signalé par BorssEAU (1962) comme très abondant au Cap-Ferret; pullule également sur l'Ile aux Oiseaux et dans les plages à arénicoles de la Hume, mais disparait à l'est de Mestras. Remonte beaucoup moins haut dans la zone intertidale que Arenicola marina. 
Melinna palmata Grube; Glemarec (1964) a montré, dans le golfe du Morbihan, que la densité de cette espèce diminuait depuis l'infralittoral non exondable $(6000$ individus au mètre carré), jusqu'au médiolittoral inférieur (400 individus au mètre carré). Le même auteur écrit: «A Arcachon, LUBET (1956) limite les herbiers de Zostera nana et hornemanniana à la partie inférieure de la zone de balancement des marées, c'est à dire entre les niveaux de basse mer de morte eau et basse mer de vive eau. Ils occupent donc la place théoriquement réservée au faciès exondable à Ampharetidae.» Melinna palmata occupe dans la baie, non seulement les mattes profondes de Zostera marina (Boisseau 1962), et le «centre des chenaux, dans les zones riches en matière organique et en poudre* (Bouchet 1962), mais encore souvent (sud de l'Ile aux Oiseaux) et de manière très dense, les herbiers superficiels de Zostera nana, où il entremêle ses tubes aux rhizomes de zostères, parfois associé à Abra alba et plus souvent à Tapes aureus.

Streblospio shrubsolii (BuchanAN); petite espèce qui échappe souvent au tri. Signalée par Boisseau (1962) dans les chenaux saumâtres de Touze, Audenge (estuaire de la Leyre), Claouey (estuaire de la rivière de Lège). C'est certainement le polychète qui remonte le plus loin en amont des estuaires, non pas tant dans la Leyre, où son extension est limitée par la pollution des eaux, mais surtout dans la rivière de Lège, où je l'ai récolté loin de l'estuaire, bien au delà de Nereis diversicolor. Il pullule également dans les réservoirs à poissons.

\section{Annélides Oligochètes}

Ce groupe fait l'objet d'un travail en cours de publication de LASSERre qui a déterminé les trois espèces citées; toutes trois ont été récoltées dans des vases saumâtres à Nereis diversicolor.

\section{Mollusques Prosobranches}

Bittium reticulatum (DA Costa), Gibbula cineraria (L.) divers Rissoa, sont des hôtes habituels de l'herbier de Zostera marina, accidentels dans l'herbier de Zostera nana. Gibbula magus (LinNé) est un eurytope, parfois abondant sur l'herbier de petites zostères.

Gibbula umbilicalis DA Costa, est lié à l'herbier de Zostera nana où il est abondamment représenté par une forme petite, à spire basse; la même espèce se retrouve dans un habitat très différent, sur les enrochements de la Vigne où de Saint-Yves, au niveau des patelles ou des plus hautes Gryphaea; elle se présente alors sous la forme d'individus de grande taille, à spire élevée, à test épais, très semblables aux exemplaires de Gibbula pennanti Prmippr, que j'ai examinés dans la collection Locard du Museum d'Histoire naturelle. B. S. Krsch, qui a eu l'amabilité d'examiner mes échantillons en 
provenance d'Arcachon, me confirme qu'il n'y a pas de différence spécifique entre les deux formes.

Hydrobia ulvae (Pennant) (= Peringia ulvae); Mars (1961), résume très exactement l'habitat de cette espèce, qui s'étend de manière continue de la basse sliklse à Zostera nana, jusqu'au sommet des schorres. C'est un herbivore qui se nourrit d'algues microscopiques. Les adultes sont surtout localisés soit dans le schorre, soit dans les vases nues à Nereis diversicolor, où ils atteignent saisonnièrement une densité de 15.000 individus au mètre carré (La Teste, novembre 1963). Les jeunes pullulent dans les ripple marks des sables nus à arénicoles. NEwELL (1962-1964), a donné d'intéressantes précisions sur le comportement écologique de cette espèce, et notamment sur la succession des phases de nage et d'enfouissement, en rapport avec le cycle de la marée. Très euryhaline, cette espèce s'étend dans toute la baie; sa densité s'accroît d'autant plus que l'on s'éloigne des passes; elle est essentiellement localisée aux plages, mais accidentellement, quelques individus peuvent en outre s'installer dans les réservoirs saumâtres en compagnie d'Hydrobia ventrosa (MTG.).

Littorina littorea L. est un eurytope qui, à Arcachon, se localise préférentiellement sur les herbiers de Zostera nana, qu'il envahit largement dans toute la baie.

Littorina obtusata (LINNÉ) (= Littorina littoralis) est surtout abondant sous les Fucus, d'où il envahit souvent l'herbier; moins euryhaline que la précédente, (Manigault 1932), cette espèce ne s'étend guère dans le fond de la baie.

\section{Mollusques Opisthobranches}

Fischer (1869), signale plusieurs opisthobranches des herbiers et crassats d'Arcachon; il serait intéressant de porter une attention systématique à ce groupe, qui est particulièrement bien représenté dans la faune des herbiers de petites zostères. D'après mes prospections, je pense que la plupart des espèces sont des hôtes plus ou moins accidentels, mais leur passage régulier à certaines saisons, surtout le printemps et l'automne, est sans doute en rapport avec une étape écologique précise.

Acera bullata O. F. Müller; cette espèce, irrégulièrement présente en été et en automne dans les herbiers sud de la baie, remonte parfois jusqu'à l'Ile aux Oiseaux, au-delà de laquelle je ne l'ai jamais récoltée. Accouplement en chaîne en août-septembre; moins abondante que Haminea navicula dont elle partage en partie l'habitat. Molander (1962), signale l'instabilité de cette espèce sur les fonds à Zostera des côtes suédoises, et rapporte cette instabilité à l'abondance variable des feuilles d'une anné à l'autre.

Cylicbna cylindracea (PenNant); accidentel dans les herbiers; provient peut être du large.

Haminea navicula (DA CosTA); envahit régulièrement les herbiers de l'Ile aux Oiseaux, surtout en septembre, où il dépose ses pontes en bordure des esteys; parfois également en Mai; certaines années (1961-1963) cette espèce remonte dans le fond de la baie jusque sur les herbiers d'Arès et Andernos. Signalé par Glemarec (1964) «de 
préférence sur les vasières intertidales» dans le Morbihan. Circule activement, à marée basse, dans la vase thixotropique, mais s'exonde rarement.

Retusa truncatula (BRUGuière); largement réparti dans tous les herbiers autour de l'Jle aux Oiseaux durant l'été 1964 (Amanieu \& Cazaux 1964); je l'ai retrouvé, moins dense, aux Hosses, en 1965. Mars (1961) considère cette espèce comme «très euryhaline, pénétrant dans la Baltique et dans la Mer Noire»; il ne semble pas que la population signalée à Arcachon en 1964, se soit véritablement installée dans la baie; d'une part elle ne s'est pas étendue au nord et à l'est de l'Ile aux Oiseaux, d'autre part elle était en nette régression en 1965.

\section{Mollusques Lamellibranches}

Abra alba (WooD); espèce liée aux fonds meubles instables des chenaux (Bouchet 1962); pullule occasionnellement dans les herbiers (février 1962, sud de l'lle aux Oiseaux).

Abra ovata (Philippi); lié surtout aux vases saumâtres à scrobiculaires. Les deux espèces Scrobicularia plana et Abra ovata constituent, avec Nereis diversicolor, l'endofaune extrêmement riche des réservoirs à poissons (voir AMANIEU 1967) parfois accompagnées par des formes lagunaires de Cardium edule.

Cardium exigunm GMELIN; accidentel, sans localisation précise. PICARD (1965) le considère comme indicateur de pollution.

Loripes lacteus (LiNNé); espèce liée aux sables fins sans couverture phanérogamique; accidentelle dans l'herbier.

Mya arenaria LINNÉ; espèce autrefois abondante dans les herbiers et les vases saumâtres de la région d'Andernos et Arès mais qui, activement recherchée pour la consommation, a disparu presque partout; est susceptible de se réinstaller, çà et là, et pullule alors rapidement tant que ses gîtes ne sont pas exploités; ainsi en 1964 se développait à Mestras, en face de la station P. 34 I, un très beau peuplement de Mya arenaria, qui s'étendit rapidement sur l'herbier, couvrant à la fin de 1964 plus d'un hectare; repéré et exploité au début de 1965, ce gisement avait disparu à la fin de l'année.

Tapes aureus (GMelin); c'est certainement le lamellibranche le plus commun dans les herbiers de Zostera nana, notamment autour de l'lle aux Oiseaux; il disparaît dans les herbiers saumâtres du nord et de l'est de la baie, au profit des myes et, plus rarement, des scrobiculaires.

\section{Arachnides Halacariens}

Je n'ai pas récolté les halacariens marins des slikkes d'Arcachon. On trouvera dans la note de ANDRE (1939) les renseignements concernant les espèces arcachonnaises littorales, saumâtres ou marines, appartenant à ce groupe. 


\section{Crustacés Ostracodes}

Ledoyer (1962) signale Asterope mariae et Xestoleberis communis sur les herbiers méditerranéens de Zostera nana. La liste des espèces récoltées à Arcachon m'a été communiquée par M. I. YAssinI qui vient de publier un travail d'ensemble sur ce groupe. Xestoleberis aurantia (BAIRD) et Leptocythere fabaeformis (G. W. MüLLER), semblent localisés aux herbiers, les autres espèces étant plus largement réparties dans les vases superficielles de la slikke.

\section{Crustacés Mysidacés}

Neomysis integer (LEACH); oxybionte, eurytherme, euryhaline, cette espèce abonde dans les flaques et les ruisselets de la région de Lège-Arès; plus rare dans l'estuaire de la Leyre. Dans le petit estuaire du courant de Lège, les bancs découvrant de sables nus, qui encombrent le lit de la rivière jusqu'au niveau de la passerelle en béton armé, sont caractérisés par la présence simultanée de Neomysis integer, qui nage en pleine eau dans les flaques, du petit tanaidacé Heterotanaïs oerstedi, qui vit au contact même du fond, et du polychète Streblospio shrubsolii qui appartient à l'endofaune; cette association est liée aux sables recouverts d'un peu de vase fine et claire, non réduite, bien oxygénée par la circulation permanente de l'eau; l'eau est entièrement douce à marée basse, inégalement salée à marée haute, atteignant parfois une salinité totale de $30 \%$.

Paramysis nowveli LABAT; pullule surtout en été dans les flaques des sables à arénicoles de l'Ile aux Oiseaux, la Hume, Gujan Mestras.

\section{Crustacés Tanaidacés}

Heterotanaïs oerstedi (KRÖYER); localisé dans l'estuaire du courant de Lège; je ne l'ai pas trouvé dans l'estuaire de la Leyre, entre le Teich et Audenge, sans doute par suite de la pollution des eaux; il est probable que des prospections plus attentives permettraient de le récolter dans la région de Certes et Lanton.

Leptochelia dubia (KRÖYER); signalé par LEDOYER (1962) dans les herbiers méditerranéens. A Arcachon, cette espèce colonise des habitats très variés dans toute la baie (AMANieu \& Cazaux 1962); elle est commune notamment dans les vases superficielles des herbiers de Zostera nana.

\section{Crustacés Isopodes}

Cyatbura carinata (KRÖYER); je donne par ailleurs des précisions sur l'écologie et le cycle de certe espèce à Arcachon (voir AManieu 1969). Cyathura carinata s'étend uniformément dans tous les faciès de la slikke, et dans une grande partie de la haute 
slikke, mais n'envahit pas le schorre. Récoltes aisées en été, par tamisage de la vase superficielle, lorsque la taille modale est maximum; sa raréfaction en fin d'été n'est qu'apparente, et tient à la difficulté de récolter alors les jeunes, de petite taille, qui composent l'essentiel de la population. Dans ses habitats préférentiels (certains herbiers, vases à scrobiculaires), elle dépasse couramment une densité de 1000 individus au mètre carré; dans les sables à arénicoles, elle ne dépasse pas 400 individus au mètre carré, mais elle reste cependant toujours plus abondante sur les sables nus des plages abritées, que sur les plages océaniques ou semi-abritées (108 individus au mètre carré à Arcachon selon Salvat 1962). L'augmentation de la densité de Cyathura carinata dans les plages abritées, peut tenir tout autant au mode abrité qu'au régime saumâtre. Considéré par l'école d'Endoume (Peres \& Picard 1964), comme caractéristique de la biocénose lagunaire euryhaline et eurytherme (avec notamment Idotea viridis, sur les feuilles des Ruppia), Cyathura carinata peuple également en abondance les réservoirs à poissons d'Arcachon.

Eurydice pulchra Leach; Salvat (1962) signale dans les sédiments meubles d'Arcachon divers Eurydice, dont Eurydice pulchra qui est l'espèce la plus commune; je l'ai récoltée, à l'exclusion de tout autre Eurydice, dans les sables légèrement vaseux des plages abritées, mais jamais en abondance.

Idotea viridis (SLABBER); déjà signalée d'Archacon par SaIvat (1962), cette espèce est largement répandue dans toute la baie, sous les Fucus, sous les entéromorphes, contre l'écorce des pignots, mais elle ne pullule réellement que dans les réservoirs saumâtres d'une part, parmi l'épifaune des herbiers du fond de la baie (La Sableyre notamment) d'autre part; ailleurs elle est accidentelle; considérée par LEDOYER (1962) et PICARD (1965) comme espèce caractéristique de la Biocénose lagunaire euryhaline et eurytherme. MatZakis (1956) en a étudié le développement postembryonnaire en méditerranée (étang de Leucate) et Howes (1939) en Angleterre.

Sphaeroma rugicauda LEACH, espèce de la haute slikke et du schorre, accidentelle dans la slikke.

\section{Crustacés Amphipodes}

Ampelisca brevicornis (A. Costa) espèce signalée par Chevreux \& Fage (1925) comme très commune dans le sable, à marée basse, en divers points de nos côtes. L'école d'Endoume (Peres \& Picard 1958, Belitan-Santini \& Picard 1963) la considère comme préférentielle, et peut être caractéristique, de la Biocénose des sables fins terrigènes. Salvat (1962) l'a récoltée tout au long de l'année «en bas de plage, dans les sédiments le plus souvent réduits de quelques estrans» et la considère avec CRAWFORd (1936) et Southward (1953) comme infralittorale. Ampelisca brevicornis pullule dans les fonds sablo-vaseux de divers chenaux d'Arcachon (récoltes Bouchet). Dans les sables intertidaux, cette espèce est généralement moins dense, mais atteint cependant 300 individus au mètre carré dans les sables à Leiochone clypeata de l'lle aux Oiseaux (juillet 1964) et 180 à la Hume (juillet 1964). Les formes intertidales me paraissent différentes, plus grandes et moins pigmentées, des formes récoltées dans les chenaux; la systématique du genre serait sans doute à préciser. 
Apberusa bispinosa (BATE); pullule généralement au printemps (mars-avril) dans les petites flaques des herbiers de Zostera nana et hornemanniana; adulte des deux sexes, toutes les femelles sont alors gestantes; non récolté en dehors de cette saison. SALVAT (1962) cite un exemplaire d'Apherusa ovalipes récolté dans les sables du Camp Américain en mars 1959; Chevreux \& Fage (1925) citent les deux espèces d'Arcachon. Ledoyer (1962) considère Apherusa bispinosa comme fréquent dans les herbiers méditerranéens de Zostera nana.

Bathyporeia elegans Watrin et Batbyporeia pilosa Lindström; SALVAT (1962) cite, dans les sédiments meubles intertidaux semi-abrités d'Arcachon, divers Haustoriidae dont Haustorius arenarius, trois Batbyporeia et quatre Urotboe. Dans les plages abritées, je n'ai jamais récolté que Batbyporeia pilosa et $B$. elegans, qui sont accidentels dans la partie supérieure des sables les plus meubles et oxygénés de la slikke. Nous retrouverons ces espèces dans certains faciès référables à la haute slikke.

Corophium insidiosum CRAwFord, Corophium sextoni CRAWFord, Corophium volutator (PALLAS); Corophium volutator est une espèce de la haute slikke, que l'on récolte accidentellement dans quelques sables vaseux à scrobiculaires. Corophium sextoni est une espèce extrêmement commune dans la baie d'Arcachon; on la récolte soit sur les pignots (Bertrand 1939, Dessenorx 1962), soit sur les bouées; elle pullule également sur des épaves varićes, échouées sur les herbiers, très souvent aussi dans les nids de Crénilabres; on ne peut considérer cette espèce comme caractéristique de l'herbier, car elle n'y occupe que des habitats très particuliers, surajoutés au biotope principal. Coropbium insidiosum est une espèce étroitement liée aux réservoirs saumâtres d'où elle envahit, parfois en masse, mais de manière accidentelle, les herbiers périphériques (AMANIEU 1967).

Dexamine spinosa (Montagu); cité comme des plus fréquents dans les herbiers méditerranéens par Ledoyer (1962). Salvat (1962) et moi même l'avons récolté à Arcachon dans les habitats les plus variés; certainement très euryhalin, il occupe les fonds des chenaux de Comprian, de la Sableyre, du Teich et la surface des herbiers qui les bordent; mais on le récolte aussi dans des biotopes beaucoup plus océaniques, à la Vigne et Arguin. Bien que largement répartie dans toute la baie, cette espèce n'est jamais abondante, les récoltes se limitant tout au plus à 1 ou 2 individus par station; j'ignore quel est son biotope préférentiel.

Erichthonius brasiliensis (DANA), Erichtbonius difformis H. MILNE EDwards; mêmes remarques que pour Corophium insidiosum.

Hyale nilssoni (RATHKE), petit Talitridae de la haute slikke, qui colonise accidentellement la slikke (dessous de Fucus). L'espèce est donnée comme sans signification précise des herbiers méditerranéens de petites zostères, par TRUE-Schlenz (1965). Giovannini (1965) a récemment précisé la systématique de cette espèce. Gammarus aequicauda Martynov; espèce du groupe Locusta déterminée par Straskraba, que je remercie vivement d'avoir bien voulu examiner de nombreux échantillons d'Arcachon; c'est le gammare le plus répandu dans toute la baie, dont il est susceptible d'occuper à peu près tous les biotopes intertidaux, saumâtres ou marins. Habitat préférentiel: réservoirs à poissons. 
Marinogammarus marinus (LEACH); les adultes des deux sexes, généralement accouplés, remontent régulièrement tous les ans au printemps, de mars à mai, sur les herbiers de l'Ile aux Oiseaux; très rare dans cet habitat en dehors de cette saison.

Melita palmata (MonTagu); espèce répandue un peu partout dans la baie d'Arcachon, surtout là où les influences saumâtres sont sensibles, mais indifférente au substrat (sables de l'horizon des sources, sous des débris, aux Abatilles, crassats saumâtres du fond de la baie). Lié à la dessalure et peut-être indicateur de pollution. Souvent signalé dans les herbiers par divers auteurs, parfois très abondant, mais irrégulièrement dans les herbiers de petites zostères à Arès et Andernos; son habitat préférentiel semble être cependant les réservoirs à poissons.

Microdeutopus anomalus (RATHKE), M. gryllotalpa DA CostA, M. stationis DellA VALLE; j'ai récolté les trois espèces sur divers herbiers à plusieurs reprises. $M$. anomalus a été signale par BERTRAND (1939) à Arcachon sur les pieux immergés; c'est en effet une espèce commune sur les pignots, d'où elle envahit accidentellement les différents herbiers. M. stationis est considéré comme une «espèce des herbiers» par SAlvat (1962), mais il est surtout lié à l'herbier de Zostera marina. M. gryllotalpa est considéré comme caractéristique des herbiers méditerranéens de Zostera nana par Ledoyer (1962) et True-Schlenz (1965); Chevreux \& Fage (1925) le citent des vasières et des marais salants (réservoirs à poissons) et des parcs à huitres; cette espèce est accidentelle sur les herbiers, alors qu'elle pullule dans les réservoirs saumâtres.

\section{Crustacés Décapodes}

Diverses crevettes (Hippolyte, Leander) pullulent dans les flaques d'herbiers. Grangon vulgaris FaBricius, l'été et surtout Palaemonetes varians (LEACH), toute l'année, occupent les flaques à salinité variable et le cours des esteys.

Upogebia pusilla (Petagna) est lié aux sables vaseux très compacts, peu saumâtres, dans lesquels il creuse ses galeries très profondes, Peres \& PICARD (1964) ont décrit, dans la biocénose des sables vaseux superficiels en mode calme, un faciès de l'Upogebia pusilla qui «qui correspond à des sédiments compactés en profondeur (où se situent les ramifications durables du terrier) et à des sédiments plus mobiles dans les quelques centimètres superficiels - (la position des terriers variant fréquemment)». Un habitat analogue se trouve à Arcachon.

\section{Insectes Diptères}

Fucellia maritima Hal. et Coelopa frigida Fall. pullulent en été sur les herbiers sableux au nord de l'Ile; Ceratinostoma ostiorum $\mathrm{H}_{\text {AL. }}$. est une espèce dont les larves sont abondantes dans les herbiers vaseux et saumâtres de Claouey et de Lanton, alors que les larves d'Ochrops sont récoltées plutôt dans les schorres sableux ou argilosableux. Outre ces formes banales, de nombreuses larves non identifiées ont été récoltées, par exemple, dans la région de Lanton et de Lège, les herbiers et les vases nues à Nereis diversicolor abritent une faune souvent très dense $(1 / 3$ de la biomasse de l'endofaune), de larves de Chironomidae et Ceratopogonidae. 


\section{LA HAUTE SLIKKE}

\section{Introduction}

Nous reconnaîtrons dans les aires référables à la haute slikke: (1) Les sables argileux à Bledius spectabilis, (2) les sables vaseux à Coropbium volutator, (3) les sables poreux à Bledius arenarius, (4) les aires envahies par Spartina maritima et (5) les microfalaises.

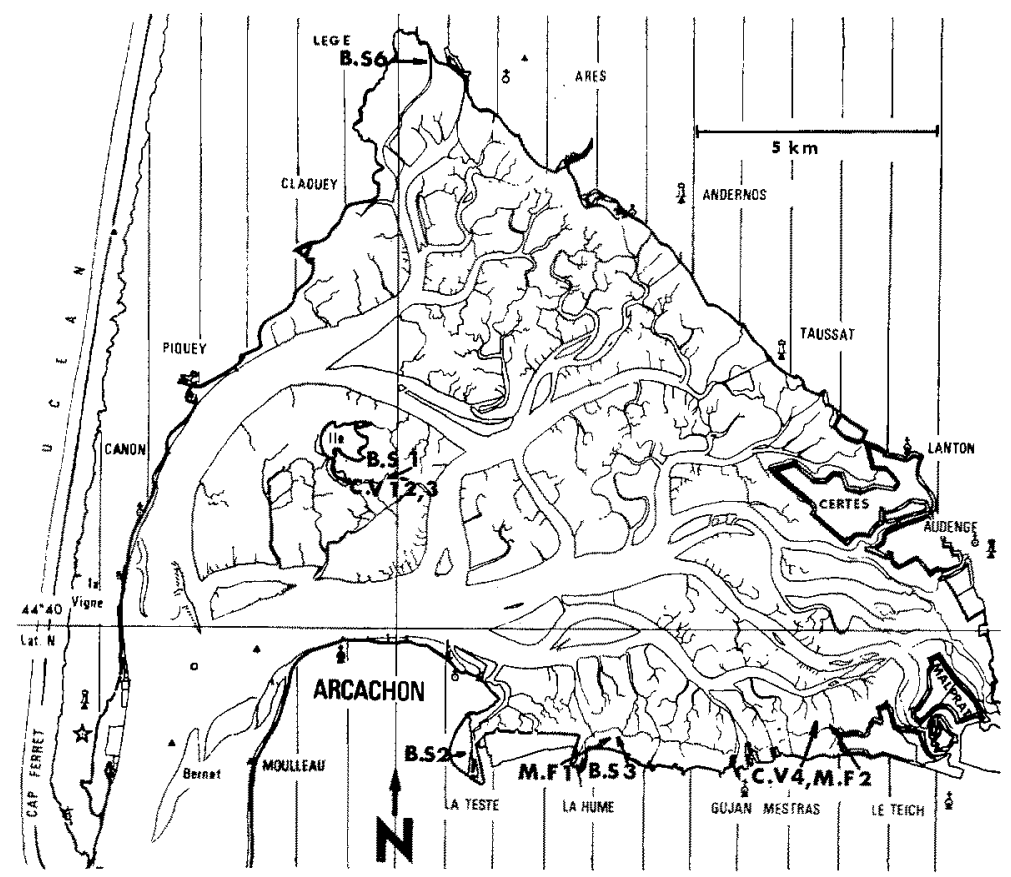

Fig. 8: Emplacement des stations prospectées dans la haute slikke. B.S. 1 à $6=$ Sables argileux à Bledius spectabilis. C.V. 1 à $4=$ Sables vaseux à Corophium volutator. M.F. 1 et $2=$ Microfalaises

Nous pensons que ces ensembles ont valeur de faciès; toutefois, comme nous l'avons déjà indiqué à propos de la slikke, seules des recherches d'écologie dynamique, qui sont le prolongement logique des résultats présentés ici, permettront de préciser la structure des biocénoses de la haute slikke (Fig. 8).

\section{Les sables argileux à Bledius spectabilis}

Les Bledius sont de petits coléoptères staphylinides, la plupart ripicoles ou marins; en particulier, Bledius spectabilis est lié à certains biotopes référables à la 
haute slikke (Fig. 9). Le faciès des sables argileux à Bledius spectabilis est caractérisé, à la fois, par sa position le long de l'estran, et par la compacité des sols qui lui correspondent; il apparaît en outre, au vu de l'analyse des contenus stomacaux de l'insecte (PAULtan 1941), que la microflore, et particulièrement les diatomées benthiques, jouent un rôle essentiel dans l'alimentation, donc dans la présence, de Bledius spectabilis.

Indépendamment des migrations de plus ou moins grande amplitude signalées par divers auteurs, Bledius spectabilis est une espèce vagile, qui effectue à marée basse
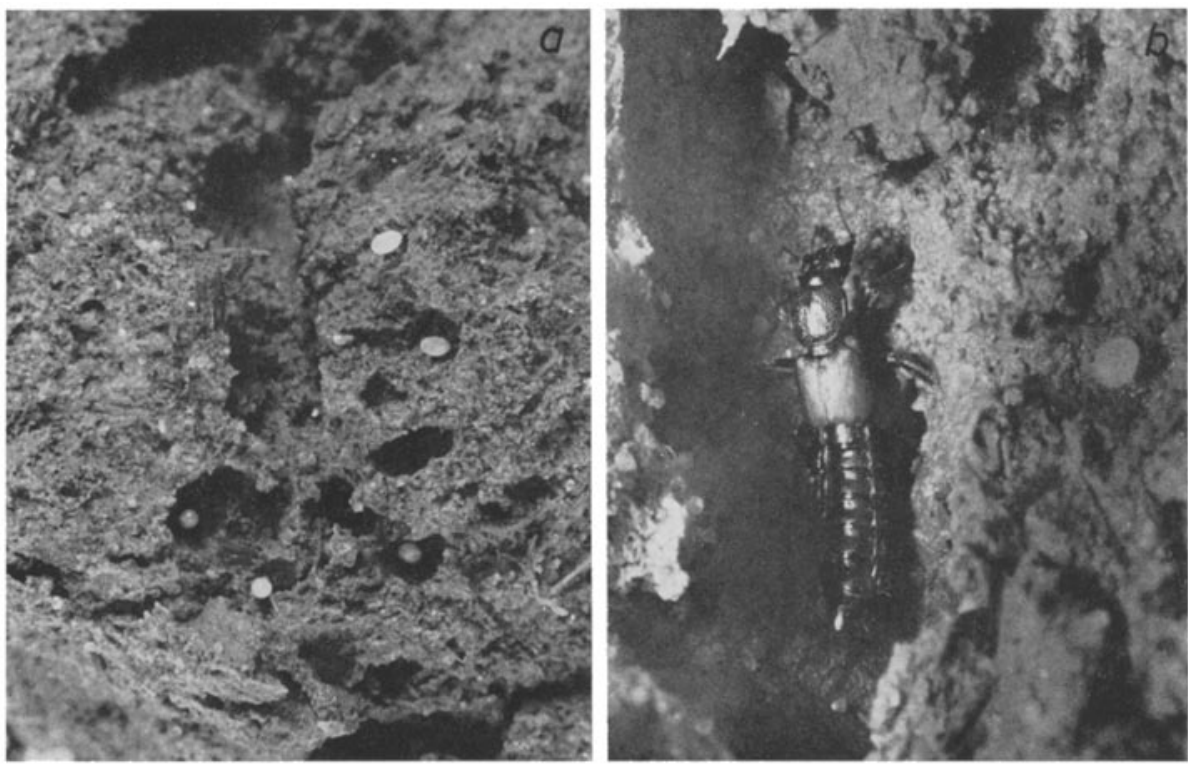

Fig. 9: Les sables argileux à Bledius spectabilis. (a) Disposition des pontes de Bledius spectabilis. Chaque oeuf est suspendu au plafond d'une logette indépendante; les logettes sont disposées en couronne autour de l'axe de la galerie principale. (b) Bleditus spectabilis, femelle occupée à pondre. L'insecte circule dans la galerie principale et vient de déposer un oeuf situé dans la logette, à droite de la photo

de petits déplacements à terre, au cours desquels il est susceptible d'envahir la majeure partie des faciès référables à la haute slikke, et même au schorre inférieur. Par contre, les galeries permanentes qu'il creuse dans le sédiment, particulièrement les galeries très structurées qu'il édifie au moment de la ponte, sont étroitement localisées à des aires de petites dimensions, parfois réduites à quelques mètres carrés, mais très dispersées. Une telle localisation implique des exigences écologique strictes, que nous préciserons dans les paragraphes suivants: dans ces aires, Bledius spectabilis représente l'espèce largement dominante et parfois unique de la macrofaune endogée; c'est à de telles aires que nous réserverons le terme de faciès des sables argileux à Bledius spectabilis. 


\section{Teneur en eau et volume en air}

Les sables argileux à Bledius spectabilis ont une teneur en eau homogène dans toute l'épaisseur du sol colonisé par l'insecte; les mesures rapportées, concernent donc uniformément la teneur en eau, depuis la surface jusqu'à $-15 \mathrm{~cm}$ environ. Cette teneur varie par contre suivant les stations, les saisons, les marées. L'étude de la variation en fonction des marées est la plus intéressante à considérer. J'ai choisi à cet effet une station (station B.S. 1 voir Fig. 8) sur l'lle aux Oiseaux, le long de la berge est d'un estey qui serpente en bas du schorre. Cette station a été retenue en raison de son homogénéité et de son importance; sur une cinquantaine de mètres de longueur, et quelques mètres de largeur, Bledius spectabilis occupe, de manière dense et uniforme, la majeure partie de la berge explorée. De janvier à mai 1961, diverses prospections ont été faites, les unes après une période de mortes eaux, les autres après les vives eaux. A chaque prospection, effectuée à marée basse, cinq échantillons de sol étaient prélevés puis ramenés et desséchés au laboratoire ( $110^{\circ}$ jusqu'à poids constant). Les chiffres donnés ci-dessous précisent la teneur moyenne des cinq échantillons et les teneurs extrêmes; la teneur en eau est toujours exprimée par rapport au poids sec du sédiment. On trouvera également indiqué dans ce tableau, les coefficients des six marées qui ont précédé la récolte; par «coefficient total» nous entendrons la somme de ces six coefficients.

\section{Tableau 9}

Teneur en eau des sables argileux à Bledius spectabilis en fonction des marées

\begin{tabular}{|cccccc|}
\hline Dates & $\begin{array}{c}\text { Coefficients des 6 marées } \\
\text { anterieures }\end{array}$ & $\begin{array}{c}\text { Coefficient } \\
\text { total }\end{array}$ & $\begin{array}{c}\text { Teneur en eauTeneur en eau } \\
\text { moyennes }(\%) \text { extrèmes }(0 / 0)\end{array}$ \\
\hline 21 janvier 1961 & $107,105,102,97,91,85$ & 587 & 64,8 & $60,1 \grave{a} 68,6$ \\
7 février 1961 & $78,76,73,69,65,61$ & 422 & 50,0 & 49 à 52,2 \\
17 février 1961 & $102,107,110,112,112,111$ & 654 & 75,5 & 71,3 à 76,9 \\
25 février 1961 & $52,46,41,38,36,37$ & 250 & 43,5 & $41,5 \grave{a} 47,2$ \\
17 avril 1961 & $104,105,105,103,100,97$ & 614 & 63,9 & 60 à 65,2 \\
26 avril 1961 & $35,34,35,38,42,47$ & 231 & 44,2 & 43,2 à 46 \\
16 mai 1961 & $91,93,94,94,93,91$ & 556 & 50 & 48,4 à 51,6 \\
\hline
\end{tabular}

Si l'on classe les teneurs en eaux en fonction des valeurs décroissantes des coefficients totaux, on obtient la série suivante: $654(75,5 \%) ; 614(63,9 \%) ; 587(64,8 \%)$; $556(50,0 \%) ; 422(50,0 \%) ; 250(43,5 \%) ; 231(44,2 \%)$.

S'il n'y a pas un rapport constant entre coefficients de marées et teneurs en eau, on voit cependant que les fortes marées s'accompagnent, dans l'ensemble, d'une teneur en eau plus élevée que les faibles marées. Ces mesures montrent, en outre, que malgré la quantité d'eau relativement importante retenue par ces sols, (la teneur en eau des

3 Divers auteurs emploient les termes de «teneur en air», et symétriquement, de «teneur en eau» pour désigner les volumes en air et en eau enfermés dans $100 \mathrm{ccm}$ de sédiment; or la «teneur en eau» définit conventionnellement, non pas un volume mais un poids rapporté à un poids, sec ou humide, de sédiment (Brajnikov, Francrs-Boeve \& Romanovsky 1943). Afin d'éviter toute confusion, je préfère employer, en ce qui concerne la porosité, les termes de «volume en air» et de «volume en eau», rapportés à un volume de sédiment. 
sables à arénicoles descend à $20 \%$ ), les sables argileux à Bledius spectabilis sont cependant loin d'être saturés par l'eau qui les inonde.

Le volume en air est variable, mais toujours élevé, et d'autant plus élevé que la densité des animaux est plus forte; dans les sables argileux à Bledius spectabilis, le volume en air mesure en effet le volume des galeries, beaucoup plus que le volume de l'air interstitiel qui est certainement très faible (Tab. 10). Sur l'lle aux Oiseaux (station B. S. 1), le volume en air atteint, ou même dépasse, $80 \%$ du volume total, le

Tableau 10

Volume en air des sols occupés par Bledius spectabilis

\begin{tabular}{|ccccccc|}
\hline Echantillons & 1 & 2 & 3 & 4 & 5 & Moyeane \\
\hline Marée haute & $63,9 \%$ & $78,3 \%$ & $61,0 \%$ & $80,0 \%$ & $75,6 \%$ & $71,8 \%$ \\
Marée basse & $62,9 \%$ & $83,4 \%$ & $64,8 \%$ & $78,9 \%$ & $83,7 \%$ & $74,7 \%$ \\
\hline
\end{tabular}

sol apparaissant alors comme criblé des galeries de l'insecte. En surface toutefois, les traces de l'activité de Bledius spectabilis sont discrètes, réduites à quelques petits tas où sont rassemblés les déblais; les ouvertures de galeries à la surface sont rares et réduites à d'étroites fissures, de telle manière qu'à marée haute l'eau ne puisse les inonder. La cohésion du sédiment, lié par la fraction argileuse, permet d'en mesurer le volume en air, sous l'eau, à marée haute; on obtient des valeurs qui ne sont que très légèrement inférieures à celles obtenues à marée basse, ainsi qu'en témoignent les échantillonnages du Tableau 10. Ces résultats prouvent nettement qu'à marée haute, les galeries de Bledius spectabilis ne sont pas ou ne sont que très faiblement inondées.

\section{Texture du sol}

Bledius spectabilis creuse ses galeries dans des sols argileux ou argilo-sableux, dans lesquels la teneur en éléments fins est variable mais généralement élevée. Sur l'Ile aux Oiseaux (station B. S. 1) le sédiment est constitué par $15 \%$ de sable fin (entre 50 et $200 \mu$ ) et $85 \%$ d'argile granulométrique (inférieux à $50 \mu$ ); à la Teste (station B. S. 2), la totalité du sédiment traverse le tamis de vide maille de $50 \mu$. A la Hume (station B. S. 3) la teneur en sable fin est de $5 \%$ et la teneur en argile de $95 \%$. Par

Tableau 11

Composition de la fraction argileuse des sols à Bledius spectabilis

\begin{tabular}{|lccccc|}
\hline Minèraux & $\begin{array}{c}\text { Ile aux Oiseaux } \\
\text { B.S. } 1\end{array}$ & $\begin{array}{c}\text { La Hume } \\
\text { B.S.3 }\end{array}$ & $\begin{array}{c}\text { Ile aux Oiseaux } \\
\text { Nereis } \\
\text { diversicolor }\end{array}$ & $\begin{array}{c}\text { La Hume } \\
\text { Nereis } \\
\text { diversicolor }\end{array}$ & $\begin{array}{c}\text { Ile aux Oiseaux } \\
\text { Corophium } \\
\text { volutator }\end{array}$ \\
\hline Illite-Muscovite & $30 \%$ & $30 \%$ & $40 \%$ & $40 \%$ & $50 \%$ \\
Kaolinite & $30 \%$ & $30 \%$ & $30 \%$ & $20 \%$ & $30 \%$ \\
Quartz & $40 \%$ & $40 \%$ & $30 \%$ & $30 \%$ & $20 \%$ \\
Gypse et $\mathrm{NaCl}$ & - & traces & traces & $10 \%$ & traces \\
\hline
\end{tabular}


contre à Mestras, dans une station de la haute slikke qui paraissait à priori convenir à 1'installation de Bledius spectabilis, j'avais été étonné de ne pas récolter cette espèce; or, à l'analyse, le sédiment s'est révélé contenir $12 \%$ de sable grossier (supérieur à $200 \mu$ ) $48 \%$ de sable fin (entre 50 et $200 \mu$ ) et seulement $40 \%$ d'argile granulométrique inférieur à $50 \mu$.

M. KLINGEBIEL, que je remercie vivement, a eu l'amabilité d'examiner à mon intention (diagramme de diffraction aux rayons $\mathrm{X}$ ) la fraction argileuse de quelques stations de Bledius spectabilis et, par comparaison, de quelques stations immédiatement voisines non peuplées par les Bledius.

La présence d'argile-minéral n'est cependant pas indispensable à Bledius spectabilis; dans l'estuaire de la Seudre (Chaillevette), l'insecte creuse ses galeries dans des vases salées, desséchées en surface, dans les quelles la fraction d'argile minéral est inférieure à $5 \%$; dans la baie même d'Arcachon, sur les bords du courant de Lège (station B. S. 6), on trouve une population installée sur des vases presque douces, constituées par des grains de quartz très fins liés par des collö̈des organiques. Ce qui compte essentiellement, ce sont les caractéristiques mécaniques du sol, qui doit être à la fois imperméable et cohérent, de manière à permettre l'édification de galeries non inondables dont les parois restent stables, et dans lesquelles les animaux circulent librement.

\section{Salinité de l'eau d'imprégnation}

L'eau qui imprègne les sols occupés par Bledius spectabilis a été extraite mécaniquement au laboratoire, par centrifugation du sédiment frais (15 minutes à 6000 tours minutes). La méthode habituelle, qui consistait à comprimer le sédiment entre les mâchoires d'un presse fruit, ne donne pas une quantité d'eau suffisante pour effectuer les dosages. Le Tableau 12 rapporte les résultats des échantillonnages effectués sur l'Ile aux Oiseaux (station B. S. 1) (moyenne et extrêmes de 5 échantillons par prise).

\section{Tableau 12}

Salinité des eaux qui imprègnent les sols à Bledius spectabilis

\begin{tabular}{|llccc|}
\hline \multicolumn{1}{|c}{ Dates } & $\begin{array}{c}\text { Coefficients des } 6 \text { marées } \\
\text { antérieures }\end{array}$ & $\begin{array}{c}\text { Coefficient } \\
\text { total }\end{array}$ & $\begin{array}{c}\text { Salinité moyenne } \\
\text { des } 5 \text { échantillons } \\
\% 0\end{array}$ & $\begin{array}{c}\text { Salinités } \\
\text { extrếmes }\end{array}$ \\
\hline 11 août 1960 & $106,104,101,96,91,85$ & 583 & 37 & 34,3 à 39,7 \\
30 août 1960 & $73,68,63,59,55,52$ & 370 & 42 & 39,5 à 44,0 \\
21 janvier 1961 & $107,105,102,97,91,85$ & 587 & 16,3 & 15,4 à 17,9 \\
25 février 1961 & $52,46,41,38,36,37$ & 250 & 18,8 & 18,0 à 20,6 \\
\hline
\end{tabular}

La salinité est plus élevée en été qu'en hiver, mais aussi après une période de mortes eaux qu'après une période de vives eaux. Sur les bords du courant de Lège (station B. S. 6) la salinité de l'eau d'imprégnation est très faible ( $5 \%$ en septembre 1964), ce qui n'empêche pas l'installation d'une population dense de Bledius spectabilis. En revanche, les espèces qui l'accompagnent habituellement dans les faciès 
qu'il nomme ont disparu, au profit du petit gastéropode Potamopyrgus jenkinsi, qui $s^{3}$ abrite dans la vase superficielle très humide.

\section{Répartition bypsométrique}

J'ai mesuré l'altitude des points culminants des aires à Bledius spectabilis à: La Hume: 6 mesures entre $+3,03 \mathrm{~m}$ et $+3,19 \mathrm{~m}$; Gujan Mestras: 12 mesures entre $+3,20 \mathrm{~m}$ et $+3,74 \mathrm{~m}$; Cap Ferret: 6 mesures entre $+3,11 \mathrm{~m}$ et $+3,25 \mathrm{~m}$; Ile aux Oiseaux: 12 mesures entre $+3,01 \mathrm{~m}$ et $+3,47 \mathrm{~m}$.

J'ai relevé l'amplitude de ces mêmes aires à: Gujan Mestras: Base d'un peuplement à $+3,22 \mathrm{~m}$, sommet du même peuplement à $+3,53 \mathrm{~m}$; amplitude $0,31 \mathrm{~m}$. Ile aux Oiseaux: Base d'un peuplement à $+2,99 \mathrm{~m}$, sommet du même peuplement à $+3,47 \mathrm{~m}$, amplitude $0,48 \mathrm{~m}$. Base d'un autre peuplement à $+2,70 \mathrm{~m}$, sommet du même peuplement à $+3,01 \mathrm{~m}$, amplitude $0,31 \mathrm{~m}$.

\section{Les sables vaseux à Coropbium volutator}

Coropbium volutator est lune des espèces la plus fréquemment citée comme représentant de la macrofaune des régions d'estuaires. Bien que cette espèce mène surtout une vie endogée, sa présence est facile à déceler grâce aux deux orifices par lesquels son tube en $U$, verticalement enfoncé dans le sol, s'ouvre librement à la surface. Comme la plupart des Corophium, C. volutator est susceptible de pulluler; par exemple Rullier (1959) écrit «... dans les marais saumâtres gagnés sur la mer (à Roscoff), les Coropbium volutator abondent au point de rendre la surface de la vase grumeleuse par leurs déjections et les orifices de leurs tubes et de laisser parfois un dépôt continu à la surface de l'eau, dépôt formé de leurs exuvies». A Arcachon je n'ai jamais observé une telle abondance; les tubes de Coropbium volutator occupent des aires de dimensions restreintes mais bien caractéristiques; contrairement aux aires sablo-argileuses de Bledius spectabilis, où les algues sont discrètes, très souvent les aires à Corophium volutator sont uniformément recouvertes par un tapis plus ou moins important de diverses Vatcheria (dont Vaucheria thuretii). La densité des tubes dépasse rarement une dizaine au décimètre carré, le plus souvent elle est de l'ordre de 3 à 4 au décimètre carré. Le terme de faciès des sables vaseux à Coropbium volutator sera appliqué exclusivement aux aires où sont édifiés les tubes. Outre cet habitat, qui est le plus caractéristique, l'espèce se rencontre sporadiquement dans les flaques saumâtres, dans les esteys, dans les réservoirs à poissons, où elle n'atteint jamais une densité élevée

\section{Teneur en eau et volume en air}

Sur l'Ile aux Oiseaux (stations C.V. 1 voir carte Fig. 8) la teneur en eau d'une aire à Corophium volutator voisine de la station B.S. 1 étudiée plus haut, a été 
mesurée dans les mêmes conditions (Tab. 13). Les deux stations ne sont pas exactement à la même altitude, les aires occupées par l'amphipode étant plus basses et plus proches de l'estey, que celles occupées par l'insecte. La teneur en eau des quinzes premiers centimètres d'épaisseur du sol, rapportée au poids sec de sédiment, est élevée $(75 \%$ à $80 \%)$ en vives eaux, et ne décroît que faiblement en mortes eaux $(73 \%$ à $78 \%$ ); la station se situe en effet aux environs de $+3,00 \mathrm{~m}$, elle est donc inondée à presque toutes les marées. Contrairement aux sables argileux à Bledius spectabilis,

Tableau 13

Teneur en eau des sables vaseux à Coroplinm volutator

\begin{tabular}{|ccc|}
\hline Localité & Strate algale & Strate sans algue \\
\hline Ile aux Oiseaux (C.V. 2) & $53,6 \%$ & $44,9 \%$ \\
Mestras (C.V. 4) & $112,76 \%$ & $63,9 \%$ \\
\hline
\end{tabular}

les sols à Coropbium volutator sont généralement stratifiés au point de vue de leur teneur en eau. Sur l'lle aux Oiseaux (station C.V.2) et à Mestras (station C.V.4), on a mesuré séparément, en septembre 1965, la teneur en eau de la strate superficielle qui correspond à l'épaisseur du sol $(2 \grave{\text { à }} 3 \mathrm{~cm}$ ) où le revêtement par les Vaucheria est suffisamment implanté pour permettre de déliter à la main un feutrage homogène, et la teneur en eau de la strate plus profonde (jusqu'à $10 \mathrm{~cm}$ environ) où se prolonge encore les tubes des amphipodes.

Le feutrage de Vaucheria forme une éponge organique qui s'imbibe rapidement d'eau à marée haute. Dans certaines stations, notamment les plus élevées, le revêtement de Vaucheria s'amenuise ou disparaît; la teneur en eau s'abaisse considérablement, la plus basse valeur notée étant de $28,7 \%$ (Ile aux Oiseaux C.V. 3, vives eaux de juillet 1961).

Le volume en air est également intéressant à mesurer. Rullier (1959) indique que l'air occupe un volume de $11,6 \%$ à $14,2 \%$ du volume total dans les sables à Corophizm de l'Aber de Roscoff; j'ai trouvé à Arcachon des chiffres un peu supérieurs (de 18 à 24\% sur l'lle aux Oiseaux), pour les mesures effectuées à marée basse, dans une station où la densité des animaux était de 11 terriers occupés au décimètre carré (C.V. 3 juillet 1961). J'ai également mesuré le volume en air à marée haute, sous l'eau; bien que l'échantillonnage soit plus difficile que pour les argiles à Bledius, la mesure conduit à des valeurs assez basses, de l'ordre de 3 à $5 \%$; il me paraît donc certain que, contrairement à ceux de Bledius spectabilis, les tubes de Corophium volutator sont en majeure partie inondés à marée haute.

\section{Texture des sols}

MoNon (1926) écrivait à propos de Coropbium volutator: «Sa localisation et son extraordinaire abondance dans la tangue ont pour cause la parfaite convenance du substratum (vase argileuse humide) à l'établissement de ses galeries.» Hart (1930) estime que: «The investigation of the habit shows that the nature of the substratum 
is the chief factor influencing the distribution of Corophium volutator.» RULLIER (1959) précise que: «. . . le biotope de Corophium volutator est donc défini par un sable très riche en particules très fines, argileuses, sans gros éléments, riche en matière organique et situé tout en haut de la plage supérieure.» La texture du sédiment d'une part, la position élevée des sols à Corophium volutator d'autre part, apparaissent en effet comme les facteurs les plus importants dans la répartition de cette espèce. A Ambleteuse, près de Wimereux, j'ai montré (AMAnIEu 1962) que les sables à Corophium volutator présentaient, du point de vue granulométrique, un premier maximum à $44 \mu$ et un second plus faible à $200 \mu$, alors que Nereis diversicolor pullule non seulement dans des sols de type analogue mais encore dans des sables grossiers, dans lesquels la fraction fine inférieure à $100 \mu$ est presque nulle, le mode se situant à $300 \mu$. A Arcachon, on a analysé pour 30 stations réparties autour de la baie, l'importance de la fraction fine inférieure à $50 \mu$, des sables fins entre 50 et $200 \mu$, des sables grossiers supérieurs à $200 \mu$. Les chiffres extrêmes sont les suivants (Tab. 14):

Tableau 14

Texture des sols à Coropbium volutator d'Arcachon

\begin{tabular}{|cccc|}
\hline Stations & Vases & Sables fins & Sables grossiers \\
& $<50 \mu$ & de 50 à $200 \mu$ & $>200 \mu$ \\
\hline Ile aux Oiseaux & $2,8 \%$ & $93,8 \%$ & $3,4 \%$ \\
Mestras & $91,7 \% \%$ & $5,2 \%$ & $3,1 \%$ \\
\hline
\end{tabular}

La teneur en sable grossier est toujours faible, alors que la teneur en vase et en sable fin est toujours élevée, mais la proportion de vase proprement dite varie considérablement. Bien qu’apparemment assez hétérogènes, ces conditions s'opposent à celles notées pour Nereis diversicolor. Les exigences de Coropbium volutator quant à la texture du sédiment sont certainement beaucoup plus strictes que celles de Nereis diversicolor qui l'accompagne souvent dans son habitat préférentiel. L'importance de la fraction fine, vases ou sables fins, dans les sols à Corophium volutator, paraît liée à la nécessité d'édifier des tubes dont les parois restent stables et cohérentes. J'ai recherché la quantité d'argile-minéral dans la fraction inférieure à $50 \mu$; cette quantité est très variable, mais généralement plus faible que dans les sables argileux à Bledius spectabilis; la fraction fine est représentée surtout par des grains de quartz liés par des collö̈des. J'ai donc préféré employer, pour les sols à Coropbium volutator d'Arcachon, le terme de sables vaseux.

\section{Salinité de l'eau d'imprégnation}

L'eau qui imprègne le sédiment a été extraite mécaniquement au laboratoire (Tab. 15). Cinq échantillons ont été prélevés sur l'lle aux Oiseaux (station C.V. 1) en août 1960 puis en février 1961. Etant donnée la faible épaisseur du revêtement de Vaucheria dans cette station, il n'a pas été fait de distinction entre strate superficielle et profonde. 
Tableau 15

Salinités des eaux qui imprègnent les sols à Coropbium volutator

\begin{tabular}{|lcccc|}
\hline \multicolumn{1}{|c}{ Dates } & $\begin{array}{c}\text { Coefficients des 6 marées } \\
\text { antérieures }\end{array}$ & $\begin{array}{c}\text { Coefficient } \\
\text { total }\end{array}$ & $\begin{array}{c}\text { Salinités } \\
\text { moyennes } \\
(\% 0)\end{array}$ & $\begin{array}{c}\text { Salinités } \\
\text { extrêmes } \\
(\% 0)\end{array}$ \\
\hline 30 août 1960 & $73,68,63,59,55,52$ & 370 & 39 & 38,3 a 40,8 \\
21 janvier 1961 & $107,105,102,97,91,85$ & 587 & 15,5 & 15,0 à 16,1 \\
\hline
\end{tabular}

Les variations saisonnières sont considérables. La salinité est un peu plus faible, et peut-être un peu plus homogène, que dans le faciès des sables argileux à Bledius spectabilis (comparer avec les valeurs rapportées pour les mêmes dates Tab. 12).

Corophium volutator et la majorité des espèces qui l'accompagnent dans le faciès qu'il nomme, s'accomodent cependant de salinités plus faibles; à Ambleteuse les sols à Corophium volutator etaient imprégnés, en mai 1961, d'une eau dont la salinité ne dépassait pas 13\% (AMANieu 1961); à la Hume, dans la baie d'Arcachon, janvier 1961, l'eau interstitielle des sables vaseux à Coropbium volutator avait une salinité totale de $10 \%$ seulement.

\section{Répartition bypsométrique}

Les habitats de Coropbium volutator, comme ceux de Bledius spectabilis, sont, dans la baie d'Arcachon, dispersés en de nombreuses aires de petites dimensions. J'ai mesuré l'altitude des points culminants de ces aires à: La Teste: 6 mesures entre $+3,26 \mathrm{~m}$ et $+3,57 \mathrm{~m}$; La Hume: 3 mesures entre $+3,00 \mathrm{~m}$ et $+3,08 \mathrm{~m}$; Gujan Mestras: 3 mesures entre $+3,00 \mathrm{~m}$ et $+3,10 \mathrm{~m}$; Lanton: 3 mesures entre $+3,20 \mathrm{~m}$ et $+3,30 \mathrm{~m}$; Cap-Ferret: (escourre du phare) 4 mesures entre $+2,83 \mathrm{~m}$ et $+3,04 \mathrm{~m}$; Ile aux Oiseaux: 9 mesures entre $+3,23 \mathrm{~m}$ et $+3,34 \mathrm{~m}$.

L'amplitude des bancs à Coropbium volutator, souvent associé à des algues du genre Vaucheria, ne dépasse pas généralement $50 \mathrm{~cm}$.

\section{Les sables poreux à Bledius arenarius}

Bledius arenarius, comme les deux espèces précédentes, occupe des aires très dispersées dans la haute slikke; cet insecte est étroitement lié à des bancs de sable propre, très poreux, inondables mais rapidement essorés après le retrait de la mer. Dans la baie d'Arcachon, les aires occupées par Bledius arenarius ne dépassent pas généralement quelques mètres carrés (Ile aux Oiseaux, littoral sud et est de la baie); toutefois, à partir de mai 1965, j'ai observé au Cap-Ferret de très vastes bancs où Bledius arenarius peuplait sur plusieurs hectares uniformément, des sables homogènes étendus au pied des dunes (côte est, abritée). Malgré leur grande dispersion autour de la baie d'Arcachon et leur aspect très varié, (ici de petits bancs de quelques mètres carrés appuyés sur la rive convexe d'un estey, là de vastes étendues de plusieurs 
hectares), les sables à Bledius arenarius sont remarquablement homogènes par leurs caractéristiques granulométriques et leur porosité élevée.

\section{Porosité, volume en air et volume en eau}

On désigne sous le nom de porosité le volume des espaces laissés entre les grains de sables; ces espaces peuvent être occupés soir par de l'air, soit par de l'eau; la porosité est exprimée en centimètres cubes pour 100 centimètres cubes de sédiment en place. La mesure de la porosité a été effectuée en s'inspirant de la méthode de Rullier (1959), qui s'applique bien aux sables étudiés ici. La porosité des sables à Bledius arenaritus est élevée, ainsi qu'il ressort d'une série de mesures effectuées en juillet-août 1963, en diverses stations: Lège: $43,5 \%$ - 48,6\% - 54,1\% - . Ile aux Oiseaux: $44,6 \%-46,0 \%-46,8 \%-47,5 \%-52,0 \%-$. La Hume: $35,3 \%-38,2 \%-$ $39,0 \%-47,5 \%-51,2 \%$ - Lugue du Cap-Ferret: $48,9 \%-48,9 \%-49,1 \%-$ $50,0 \%-51,3 \%-60,1 \%-$.

Ces chiffres témoignent de l'arrangement cubique des grains de sable (voir à ce sujet Davant \& Salvat 1961 et Renaud-Debyser 1963); dans certains cas (porosité supérieure à $50 \%$ ), on doit en outre admettre que l'on a faire à des sables alvéolaires, du type de ceux étudiés par Baudorn (1949 à 1954).

Etant donnée la perméabilité élevée des sables à Bledius arenarius, il serait de peu d'intérêt de préciser leur teneur en eau, essentiellement variable suivant l'heure de la marée. En revanche, il est intéressant de suivre la manière dont décroît, pendant la durée de l'exondation, le volume en eau au profit du volume en air. Davant \& SALVAT (1961) ont confirmé par quelques mesures que, sur des sables de haut de plage à la Vigne (baie d'Arcachon), pour une porosité totale restant inchangée entre deux marées consécutives, le volume en air croissait aux dépens du volume en eau pendant toute la durée de la basse mer.

Une série de mesures effectuées en août à Lège, dans des sables à Bledius arenarius a apporté les résultats suivants (Tab. 16):

Tableau 16

Mesures effectuées dans des sables à Bledius arenarius

\begin{tabular}{|lccccc|}
\hline Durée après l'émersion & $0.30 \mathrm{~h}$ & $1.00 \mathrm{~h}$ & $2.00 \mathrm{~h}$ & $3.00 \mathrm{~h}$ & $4.00 \mathrm{~h}$ \\
\hline Volume en air $(\%)$ & 14,4 & 23,2 & 31,0 & 35,8 & 36,0 \\
Volume en eau $(\%)$ & 34,2 & 25,1 & 17,4 & 12,9 & 12,5 \\
Porosité totale $(\%)$ & 48,6 & 48,3 & 48,4 & 48,7 & 48,5 \\
\hline
\end{tabular}

Les échantillons prélevés en une même station et, aux différentes heures, en des points aussi proches que possible, présentent une porosité totale remarquablement constante, les faibles écarts notés paraissant même inférieurs à ce que l'on aurait pu attendre des imprécisions expérimentales. En revanche, d'heure en heure, le volume en eau décrô̂t au profit du volume en air; pendant les premières heures, la perte d'eau est rapide, en rapport surtout avec l'écoulement par perméabilité; au bout de 
trois heures environ, la perte d'eau est beaucoup plus lente et sans doute imputable essentiellement à l'évaporation. La courbe Figure 10 donne une image plus parlante de ce phénomène. Il serait intéressant de connaître le volume en air du sédiment pendant l'immersion; celà n'est pas réalisable expérimentalement; sous l'eau, et même pendant la première demi-heure qui suit l'émersion, la structure du sable est trop

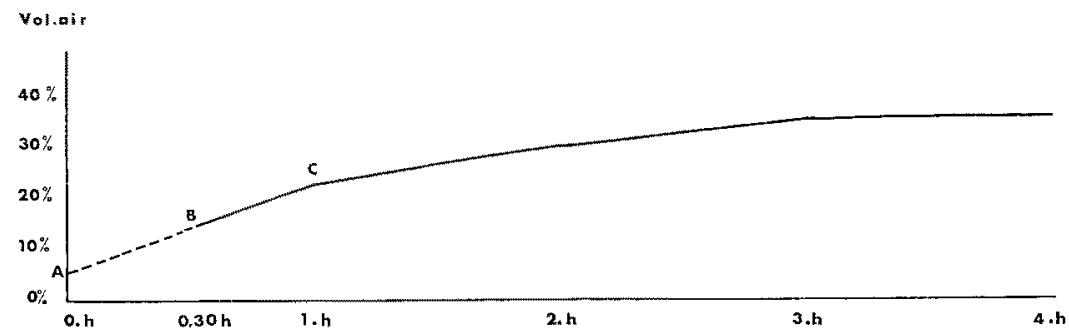

Fig. 10: Augmentation du volume en air dans les sables à Bledius arenarius au long de l'émersion

fragile pour permettre un prélèvement correct; on a cependant une certaine idée de ce volume en prolongeant linéairement vers la gauche le segment de droite $\mathrm{BC}$; la teneur en air au moment de l'immersion est au moins égale à l'ordonnée du point $\mathrm{A}$; cette teneur en air sous-immersion, évaluée indirectement, est faible $(5,6 \mathrm{ccm})$; cependant Bledius arenarius ne s'échappe pas du sable lors de l'arrivée de l'eau; il s'abrite dans ses galeries, se réfugiant dans de petits espaces étanches à l'eau, où il attend le retrait de la mer. Dans l'exemple choisi, le volume en eau après $4.00 \mathrm{~h}$ d'émersion est relativement élevé $(12,5 \mathrm{ccm})$; j'ai trouvé en d'autres circonstances des chiffres plus bas (jusqu'à $5 \mathrm{ccm}$ ) mais cependant Bledius arenarius ne peuple pas des sables qui s'asséchent totalement à marée basse.

Cep Ferre?

ife

Lège

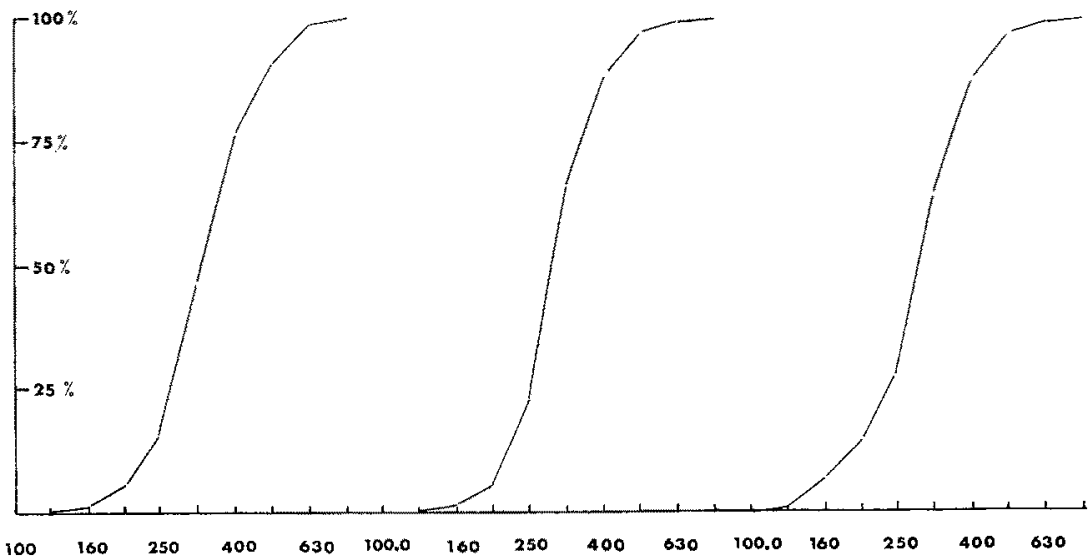

Fig. 11: Courbes granulométriques cumulatives de sables à Bledius arenarius. 3 stations: Cap Ferret, Ile aux Oiseaux et Lège 
Texture des sols

La Figure 11 représente les courbes cumulatives pondérales, en coordonnées semilogarithmiques, obtenues par tamisage (sur série de tamis AFNOR), des sables à Bledius arenarius provenant de diverses stations de la baie d'Arcachon. Nous soulignerons l'absence de particules à grains très fins inférieurs à $50 \mu$; les sables poreux à Bledius arenarius sont des sables propres, bien classés, dont le diamètre médian est de l'ordre de 250 à $300 \mu$, un peu plus grossier comme l'on devait s'y attendre, vers les passes que dans le fond du bassin. La similitude de ces sables, même lorsqu'ils proviennent de stations aussi éloignées que Lège et le Cap-Ferret (plus de $15 \mathrm{~km}$ ), tient à l'homogénéité du stock disponible dans l'ensemble de la Baie, beaucoup plus sans doute que de l'attachement préférentiel de Bledius arenarius à un type de sable de granulométrie constante.

En conclusion, les prospections effectuées dans la baie d'Arcachon permettent de préciser l'attachement de Bledius arenarius à des sables propres, de porosité élevée, non soumis à de trop longues immersions, bien oxygénés.

\section{Position bypsométrique}

Seules quelques stations de l'Ile aux Oiseaux ont été nivelées, elles culminaient entre 3,00 et $3,50 \mathrm{~m}$.

\section{Les peuplements}

Bledius arenarius est généralement le seul habitant des sables qu'il nomme; rarement j'ai noté la présence d'autres insectes: Diglossa submarina FArRM, Cillenus lateralis $\mathrm{S}_{\mathrm{AM}}$. Toutefois, il est un voisinage assez constant entre Bledius arenarius d'une part, Batbyporeia elegans et Batbyporeia pilosa d'autre part; ces deux amphipodes ont déjà été signalés à Arcachon par Salvat (1962); tandis que Batbyporeia pilosa est abondant «dans la partie inférieure de l'étage mésolittoral» des sables des plages semi-abritées de la Vigne, Bathyporeia elegans a été récolté en un seul exemplaire au banc de la Vigne. Leloup \& Konietzko (1956) dans le Bas-Escaut, ont signalé les deux espèces dans des sables saumâtres, $B$. pilosa étant beaucoup plus abondant que $B$. elegans. Dans les sables très dessalés de l'estuaire de la rivière de Lège, Batbyporeia pilosa est également beaucoup plus abondant que B. elegans. Les sables poreux à Bledius arenarius sont dispersés dans cette région en d'innombrables petits dômes déposés sur la rive convexe des esteys; l'insecte occupe la partie supérieure du dôme et les amphipodes la partie basse plus humide; il s'agit cependant de dépôts de sable homogène, ayant notamment même porosité et même granulométrie, seul diffère le volume en eau qui, à marée basse, descend à 10-12\% pour le sommet, alors que pour la base il n'est jamais inférieur à $25 \%$. J'ai retrouvé ces sables à Bledius arenarius et Batbyporeia dans la majorité des stations explorées dans la baie d'Arcachon (La Hume, l'Ile aux Oiseaux, lugue du Cap-Ferret); plus rarement 
(La Hume, l'Ile aux Oiseaux), lorsque le sous-bassement sableux devient plus compact et s'enrichit en vase, les Batbyporeia disparaissent au profit de Coropbium sp. et de Cyatbura carinata.

\section{Les peuplements de Spartina maritima}

Les phytosociologues reconnaissent dans la haute slikke une association caractéristique décrite sous le nom de Salicornieto-Spartinetum (Warming) Br.-BL. et DE LEEUw. Les spartines d'une part, les salicornes d'autre part, occupent des aires distinctes, parfois séparées, parfois imbriquées. Sur le terrain, les faciès que nous avons précédemment décrits (sables argileux à Bledius speciabilis, sables poreux à Bledius arenarius, sables vaseux à Coropbium volutator) apparaissent généralement comme des enclaves plus ou moins dispersées au milieu des peuplements de spartines; les conditions marégraphiques sont donc identiques pour les uns et les autres. Le nivellement des spartines, et particulièrement de leur base, donne une bonne idée d'ensemble de la position hypsométrique de la haute slikke. Ce nivellement est d'autant plus intéressant à considérer que d'une part, les spartines s'étendent souvent de manière homogène sur de vastes aires qu'il est donc possible de niveler en continu, et que d'autre part, faciles à identifier sur le terrain, elles se prêtent bien à des mesures rapides et nombreuses. De telles mesures nous ont en outre amené incidemment à mieux comprendre la position et la signification du Juncetum maritimi.

\section{Répartition bypsométrique de Spartina maritima à Arcachon}

Durant l'été 1963,514 points marquant la limite supérieure ou inférieure de Spartina maritima ont été nivelés autour de la baie d'Arcachon. Les résultats de ces relevés sont résumés dans le Tableau 17. Dans les flaques d'eau stagnante, les spartines les plus élevées atteignent $+3,79 \mathrm{~m}$ à Gujan, $+3,84 \mathrm{~m}$ à Certes, $+3,67 \mathrm{~m}$ à l'Ile aux Oiseaux.

\section{Tableau 17}

Limites supérieures et inférieures de Spartina maritima

\begin{tabular}{|lccc|}
\hline \multicolumn{1}{c}{ Localités } & $\begin{array}{c}\text { Limites } \\
\text { inférieures }(\mathrm{m})\end{array}$ & $\begin{array}{c}\text { Limites } \\
\text { supérieures }(\mathrm{m})\end{array}$ & $\begin{array}{c}\text { Amplitudes } \\
(\mathrm{m})\end{array}$ \\
\hline La Teste & $+2,95$ & $+3,72$ & 0,77 \\
La Hume & $+2,92$ & $+3,51$ & 0,59 \\
Gujan & $+2,83$ & $+3,50$ & 0,67 \\
Mestras & $+2,70$ & $+3,70$ & 1,00 \\
Cantarane & $+2,75$ & $+3,48$ & 0,73 \\
Malprat & $+2,83$ & $+3,30$ & 0,47 \\
Certes-Lanton & $+2,95$ & $+3,62$ & 0,67 \\
Lège-Arès & $+2,68$ & $+3,35$ & 0,67 \\
Ile aux Oiseaux & $+2,86$ & $+3,51$ & 0,65 \\
\hline
\end{tabular}




\section{Remarques}

(1) En ce qui concerne la base du peuplement, la plus basse cote notée est de $+2,68 \mathrm{~m}$ à Lège et la plus élevée de $+2,95 \mathrm{~m}$ à Lanton; la frange inférieure des spartines se distribue donc, autour de la baie d'Arcachon, le long d'une ligne dont le niveau, assez constant, oscille entre les cotes $+2,68$ et $+2,95$ (soit $27 \mathrm{~cm}$ d'amplitude); le sommet du peuplement quant à lui, oscille entre $+3,30$ à Malprat et $+3,72$ à la Teste (soit $42 \mathrm{~cm}$ d'amplitude) mais peut remonter jusqu'à $+3,84 \mathrm{~m}$ (Certes), dans des dépressions où l'eau ne s'écoule pas à marée basse; ce cas excepté, la zone d'extension des spartines se situe en définitive entre les cotes extrêmes $+2,68 \mathrm{~m}$ (frange inférieure à Lège) et $+3,72 \mathrm{~m}$ (sommet du peuplement de la Teste), avec par conséquent une amplitude totale de $1,04 \mathrm{~m}$.

(2) Les spartines débutent généralement par des peuplements monospécifiques, à recouvrement dense, qui ne s'étendent guère au-delà de 3,40 m. Ensuite, le peuplement initial se dégrade au fur et à mesure que s'y ajoutent diverses phanérogames du schorre; il y a ainsi un recouvrement très net, sur une hauteur de 30 à $40 \mathrm{~cm}$, entre les peuplements propres à la haute slikke et les végétaux du schorre, qui prendront leur pleine extension à un niveau supérieur.



Fig. 12: Répartition des Spartines dans la Baie de Cantarane 
(3) Une fraction non négligeable du peuplement des spartines empiète sur la zone inondée à toutes les marées; en effet, les spartines descendent d'une manière non exceptionnelle à $+2,80 \mathrm{~m}$ ou même $2,70 \mathrm{~m}$, alors que les hautes mers des plus faibles marées atteignent au moins le niveau $+2,95 \mathrm{~m}$.

(4) La base du peuplement des spartines se situe à un niveau voisin du sommet du peuplement des zostères; à plusieurs reprises nous avons en effet noté que les deux peuplements sont contigus. Souvent (Cantarane, Certes, Lège), les circonstances topographiques locales, qui favorisent une certaine rétention d'eau au delà de l'heure normale de basse mer, conditionnent l'apparition d'un faciès de vase nue à Nereis diversicolor, qui vient s'intercaler entre spartines et zostères; c'est souvent au détriment du peuplement des spartines, dont la base est délaminée, que se développe ce faciès; en revanche, on ne note pas dans ces cas qu'il y ait un relèvement du sommet du peuplement des spartines, qui culmine à une hauteur normale. Les nivellements de la baie de Cantarane illustrent cette remarque:

Au nord des réservoirs de Cantarane s'est constituée une petite baie (Fig. 12) ouverte vers l'ouest, fermée au nord par un schorre long de 500 mètres et large de 50 , fermée également à l'est et au sud par les digues des réservoirs. A l'entrée de la baie, la base des spartines s'établit à la cote $+2,75 \mathrm{~m}($ D.N. 1) et $+2,80 \mathrm{~m}$ (D.N. 13); le sommet du peuplement culmine à $+3,48 \mathrm{~m}$ (D.N. 12). D'est en ouest, le sommet du peuplement reste horizontal (de + 3,48 m D.N. 12 à + 3,46 m D.N. 8). En revanche, en ce qui concerne la base de ce peuplement on note que: (a) à l'intérieur de la baie elle se relève d'W. en E. de + 2,80 m (D.N. 13) à + 3,46 m (D.N. 8). Au fond de la baje, les spartines sont réduites à quelques pieds situés à la cote $+3,46 \mathrm{~m}$; tout le peuplement a été délaminé à partir de sa base par les vases à Nereis diversicolor; (b) à l'extérieur de la baie au contraire, la base du peuplement de spartines ne se relève que faiblement, de $+2,78 \mathrm{~m}$ (D.N. 11) à $+2,96 \mathrm{~m}$ (D.N. 9).

De même, dans la baie de Lanton, de la pointe de Branne au port de Lanton, le



Fig. 13: Répartition des Spartines et des Zostères autour des rèservoirs de Certes. (A) Contact Zostères - sables; les Zostères culminent à $+2,40 \mathrm{~m}$. (B) Contact sables - talus de la digue. (C) Contact Zostères - spartines à $+2,95 \mathrm{~m}$. (D) Contact Zostères - vases; les Zostères culminent $\grave{a}+3,00 \mathrm{~m}$. (E) Contact vases-spartines; les vases culminent à $+3,50 \mathrm{~m}$ et les spartines descendent jusque vers $+3,20 \mathrm{~m}$ 
sommet du peuplement des spartines reste stable $(+3,60 \mathrm{~m}$ à Branne et $+3,62 \mathrm{~m}$ à Lanton), alors que la base se relève progressivement de l'entrée de la baie $(+2,95 \mathrm{~m}$ à Branne) jusqu'au fond de celle-ci $(+3,20 \mathrm{~m}$ à Lanton). La frange inférieure délaminée est colonisée par les vases molles à Nereis diversicolor.

\section{Regression des spartines au profut de Juncus maritimus dans la région de Lège}

Dans la région de Lège-Arès, les spartines culminent au maximum à $+3,35 \mathrm{~m}$, souvent même elles sont confrontées, dès $+3,00 \mathrm{~m}$, à Juncus maritimus, qui s'étend de manière monotone sur des vases consolidées, également envahies par Nereis diversicolor. Une série de dosage de salinité des eaux interstitielles, dont les prélèvements ont été effectués en juin et septembre 1964 puis en mars 1965, aux stations indiqueées Figure 14, est rapportée sur le Tableau 18. Les valeurs figurées sur ce tableau montrent que les salinités des eaux interstitielles varient suivant les stations, les profondeurs, les saisons. Toutefois certaines considérations générales apparaissent clairement.

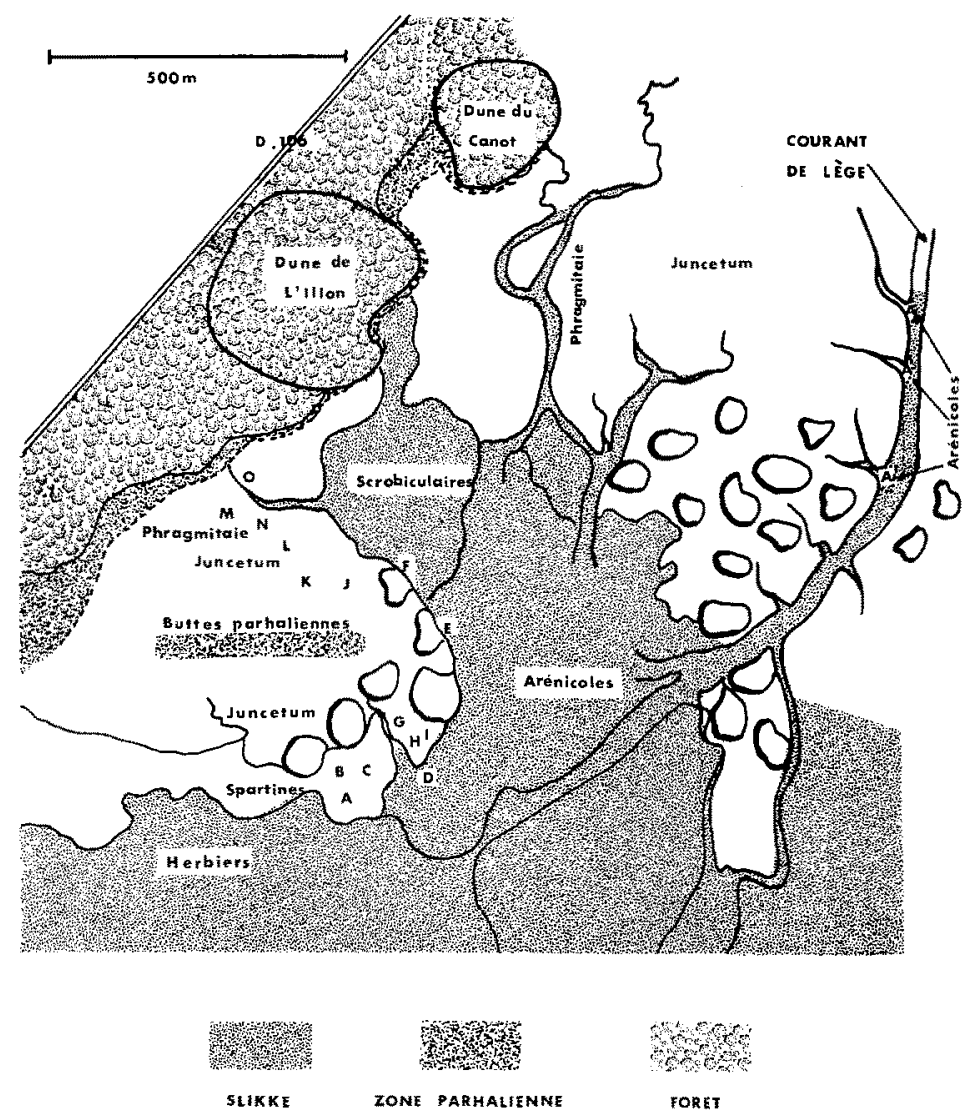

Fig. 14: Région de Lège. Répartition des différents faciès. Emplacement des stations 
(1) Au milieu des peuplements de spartines, la salinité des strates profondes cst généralement aussi élevée ou même plus élevée que dans les strates superficielles ou les flaques. Il n'y a pas, à proximité de la surface, de strate de résurgence, sauf en mars, époque de la remontée de la nappe phréatique, et en bordure supérieure du peuplement. (2) Dans les peuplements de Juncus au contraire, la salinité décroît généralement avec la profondeur, ce qui est en rapport avec la proche circulation d'une nappe phréatique qui apparaît à $-50 \mathrm{~cm}$ de la surface. (3) En bordure des roseaux, on trouve toujours une nappe souterraine, dont la profondeur est variable suivant les saisons, et dont la salinité est nulle en mars mais peut-être élevée en septembre.

Il apparaît en définitive que, dans la région de Lège-Arès, les spartines se tiennent à la limite de la zone où leurs racines restent hors d'atteinte du contact direct avec les eaux douces souterraines, d'origine continentale. Leur limite d'extension vers le haut n'est plus en rapport avec le niveaux hypsométrique mais directement avec la salinité des eaux interstitielles.

Comparativement, des dosages analogues ont été effectués sur les plages sud de l'lle aux Oiseaux. Les résultats sont monotones, en particulier dans la traversée des faciès à Spartina maritima de la haute slikke. On n'a jamais observé de nappe de résurgence; la salinité est généralement un peu plus élevée au niveau des racines $(-15 \mathrm{~cm})$ qu'en surface. Les écarts suivant les saisons sont importants; la salinité au niveau des racines est de l'ordre de 35 à $36 \%$ en septembre, 30 à $33 \%$ en juin et seulement de 16 à $17 \%$ en mars; il n'y avait ni roseaux ni joncs dans le site exploré; les eaux interstitielles de la partie superficielle du sol sont certainement peu influencées durant la majeure partie de l'année par les nappes douces, trop profondes. Il ne fait aucun doute que, sur l'lle aux Oiseaux, la limite d'extension des spartines vers le schorre, ost uniquement en rapport avec le niveau hypsométrique.

\section{Conclusion}

La strate dans laquelle dominent les faciès référables à la haute slikke est susceptible d'être colonisée par plusieurs phanérogames.

La zone d'extension de Spartina maritima est réglée par des facteurs marégraphiques d'une part, par la présence éventuelle d'une nappe d'origine continentale, circulant à proximité de la surface du sol, d'autre part. La frange inférieure des spartines est délaminée, sans qu'il y ait relèvement d'ensemble du peuplement, lorsque les circonstances topographiques locales retardent l'écoulement normal de l'eau à marée basse. La frange supérieure s'élève normalement, lorsque les apports souterrains d'eau douce sont faibles; son extension est au contraire précocement limitée lorsque, au moins de manière saisonnière, la nappe phréatique douce circule à proximité immédiate de la surface du sol.

Juncus maritimus est moins halophile, les aires où cette espèce s'étend de manière dense et constante ne sont pas zonées en fonction de la marée. Sur l'Ile aux Oiseaux, le Juncetum maritimi se situe en position élevée, il n'est alors inondé que par les très fortes marées; dans la région de Lège Arès au contraire, le Juncetum maritimi empiète largement sur la haute slikke; son extension est alors en rapport avec la présence 


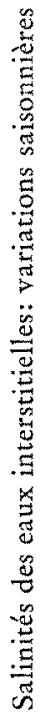

政

की

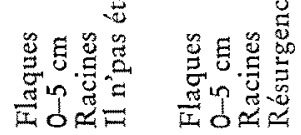



sins

U

氙

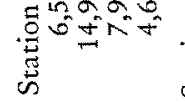

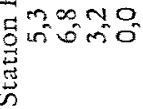

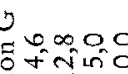

焉

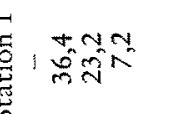

通苟

है

TON

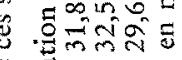

है

0

$\sin \infty$

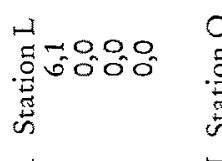

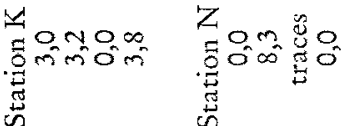

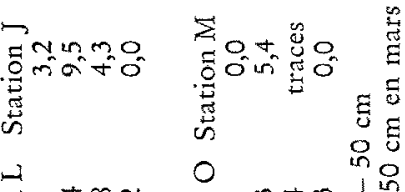

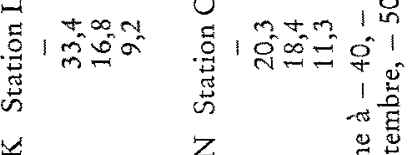


हूँ 品 동

$1 \sum_{0}+0$ in 8


U है

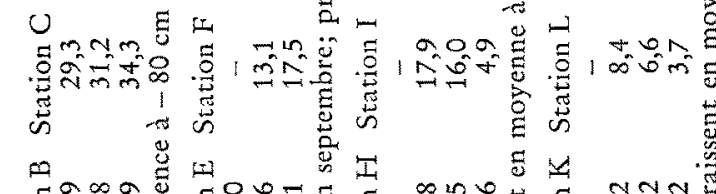

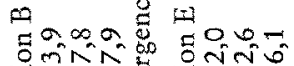

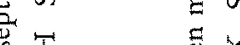

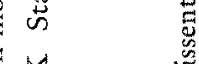

in

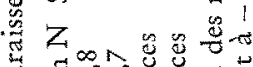

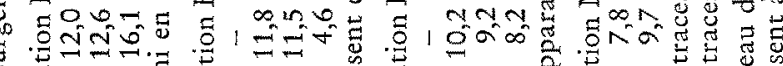

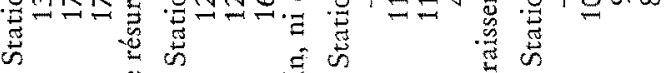

कि



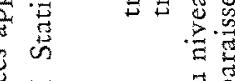

i

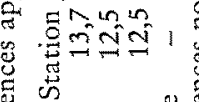

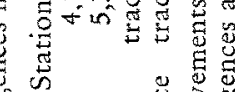
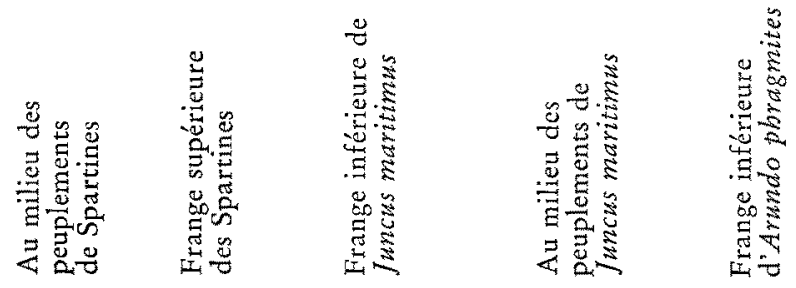
d'une nappe d'eau dessalée, et même saisonnièrement douce, qui, imprègne le sol à une faible profondeur. Cette nappe, elle même liée à la proximité des dunes, entretient une humidité importante du sol jusqu'à un niveau élevé, au moins $+3,75 \mathrm{~m}$, permettant une large extension de Nereis diversicolor.

\section{Les microfalaises}

La marge maritime des schorres est très généralement marquée par un abrupt ou microfalaise, dont l'amplitude est liée au mannage. Ces microfalaises ont été interprétées, soit comme le témoignage d'un passé géologique récent (FRANCIS BoEUF: constitution d'un dépôt lors de la plus grande extension de la transgression flandrienne, exondé à la suite de la faible régression post-flandrienne), soit comme résultant du jeu des phénomènes actuels. JACQuet (1949) dans la Manche et Verger (1956) dans l'anse de l'Aiguillon, ont donné des exemples de l'édification actuelle de schorres et de leurs microfalaises.

Ces microfalaises ont-elles un intérêt bionomique? Deux points les concernant méritent d'être examinés; leur position d'une part, l'originalité de leur peuplement d'autre part.

En ce qui concerne la position des microfalaises, JACQUET (1949) écrit: «Dans le havre de Régneville, les marées hautes de mortes eaux ordinaires arrivent ... à une hauteur seulement de $9,20 \mathrm{~m}$ au-dessus du zéro de la carte marine. Or, la bordure externe du pré salé est à une hauteur de $12,82 \mathrm{~m}$ au-dessus de ce zéro, et la ligne de $11,82 \mathrm{~m}$ suit l'abrupt du schorre...» VERGER (1956) estime de même que: «le plus souvent, la microfalaise se trouve partiellement ou même entièrement à un niveau plus élevé que celui de la solution de continutité entre le schorre et la slikke, au sens de J. Massart, C'est à dire que sa base se situe au-dessus des hautes mers de morte eau. Topographiquement, les microfalaises séparent donc la haute slikke du schorre et constituent à ce titre un bon repère bionomique. Dans la baie d'Arcachon, le faible marnage d'une part, le mode abrité des plages d'autre part, favorisent peu la formation des microfalaises qui sont discrètes; souvent il n'y a pas une seule mais deux microfalaises successivement étagées.

A la Hume (M.F. 1), on observe un premier talus dont la base se situe $a+2,91 \mathrm{~m}$ et le sommet à $+3,03 \mathrm{~m}$; bien que de faible amplitude, ce talus est nettement marqué et sépare la slikke de la haute slikke; il est confronté d'une part à des sables monotones à arénicoles, d'autre part à une aire où les touffes de spartines séparent des sables argileux à Bledius spectabilis qui s'élèvent en pente douce jusqu'à la cote $+3,19 \mathrm{~m}$; cette cote marque la base d'un second talus dont le sommet culmine à + 3,47 m; ce second talus sépare la haute slikke du Puccinellietum qui la surmonte; le Puccinellietum repose ici sur un substrat vaseux, humide, en partie occupé par les dernières spartines auxquelles se mêlent d'autres phanérogames. A Mestras (M.F. 2), la haute slikke se raccorde à la slikke par deux petits talus entre $+2,80 \mathrm{~m}$ et $+3,20 \mathrm{~m}$; elle s'étale ensuite en pente douce jusqu'au pied d'une microfalaise haute, dont la base se situe $\grave{a}+3,56 \mathrm{~m}$ et le sommet $\grave{a}+4,38 \mathrm{~m}$; au delà commence le schorre tabulaire typique, à sol ferme, à recouvrement phanérogamique dense. 
La base de la microfalaise haute de Mestras se situe au dessus $(+3,56 \mathrm{~m}) \mathrm{du}$ sommet de celle de la Hume $(+3,47 \mathrm{~m})$. La présence d'une haute slikke et de la microfalaise qui la limite, n'est donc que faiblement liée aux conditions marégraphiques, mais beaucoup plus aux facteurs pédologiques et aux conditions locales de l'érosion ou du dépôt; l'extension des végétaux, et surtout de Spartina maritima qui croît au dessus de l'abrupt à la Hume mais non à Mestras, est beaucoup plus en rapport avec les conditions marégraphiques.

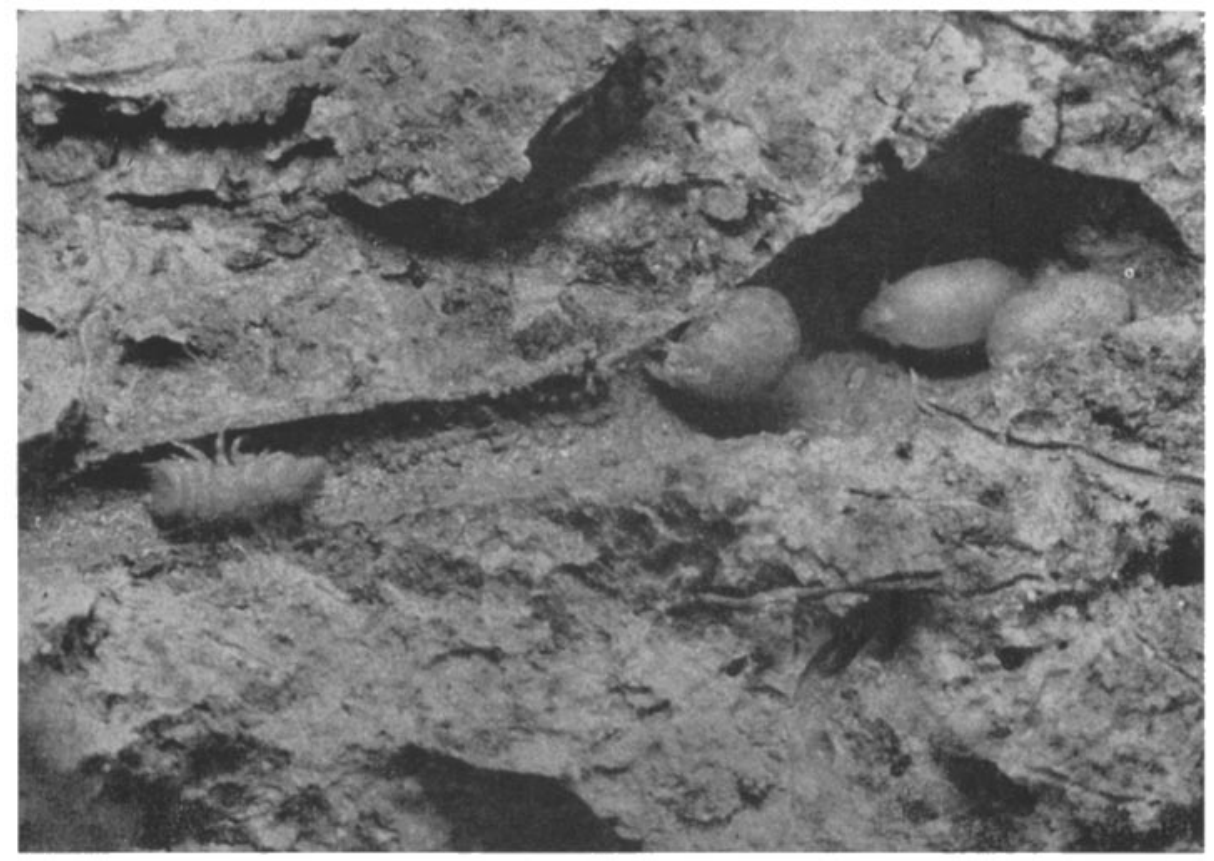

Fig. 15: Un «nid» de Paragnathia formica. A droite une femelle et deux pranizes au fond de la logette. A gauche le mâle s'échappe par le couloir d’accès qui va de la surface du sol à la logette

Sur l'tle aux Oiseaux, les microfalaises qui marquent la base du Puccinellietum s'étendent en moyenne entre $+2,80 \mathrm{~m}$ (base) et $+3,40 \mathrm{~m}$ (sommet); elles séparent, comme à la Hume, une haute slikke argileuse à Bledius spectabilis, d'un schorre inférieur incliné, partiellement envahi par les plus hautes spartines. Bien que mal zonées, il apparaît en définitive que les microfalaises séparent des aires référables à la haute slikke d'une part, à recouvrement faible et discontinu, et des aires référables au Puccinellietum, à recouvrement dense, d'autre part; suivant la position et l'amplitude des microfalaises, la haute slikke est confrontée soit à un schorre humide, en pente douce, où s'éteignent les dernières spartines (La Hume, l'Ile aux Oiseaux), qui correspond au schorre inférieur ou «marge maritime du schorre» de VERGER (1956), soit directement à un schorre tabulaire ferme, à recouvrement végétal dense et homogène (Mestras), qui correspond au schorre supérieur ou «schorre typique» de VERGER. 
En ce qui concerne leur peuplement, les microfalaises ont été parfois présentées comme un faciès individualisé, abritant une faune originale; nous ne partageons pas ce point de vue. Il est certain que quelques espèces, par exemple l'isopode Paragnatbia formica (Fig. 15), ou les coléoptères du genre Heterocerus, présentent une exceptionnelle densité sur le front des microfalaises; mais si l'on en fait un relevé faunistique plus attentif, on constate que les microfalaises abritent en réalité toutes les espèces de la haute slikke et une bonne partie des espèces vagiles du Puccinellietum; il nous paraît plus exact de considérer les microfalaises comme une frontière, un «écotone» entre deux biotopes distincts; l'originalité de leur peuplement réside dans sa richesse et sa densité, non dans sa spécificité.

\section{Conclusions sur la haute slikke}

Malgré la diversité et la dispersion des faciès qui lui sont référables, la haute slikke constitue un ensemble homogène, doué d'une originalité propre.

\section{Les conditions édaphiques}

Au point de vue hypsométrique, les faciès référables à la haute slikke s'étendent au plus entre $+2,70$ et $+3,70 \mathrm{~m}$. Au contact du Puccinellietum, la haute slikke se localise habituellement dans une zone plus étroite entre $+3,00$ et $+3,40 \mathrm{~m}$. Son extension de part et d'autre de ces niveaux préférentiels, particulièrement au dessus de $+3,40 \mathrm{~m}$, est en rapport avec la présence d'une nappe souterraine d'origine continentale, circulant à proximite de la surface du sol. Etroitement liées à la structure des sols, les aires de la haute slikke se présentent sous la forme d'indentations irrégulières, pénétrant plus ou moins profondément dans le schorre inférieur.

Contrairement à ce que l'on observe dans la slikke, où le volume en air des sédiments à marée basse est nul (cas général), ou faible (certains sables à arénicoles), le volume en air des sols de la haute slikke est toujours élevé à marée basse, élevé (sables argileux à Bledius spectabilis) ou non, (sables vaseux à Corophium volutator) à marée haute. Ce volume en air est lié soit à la présence de galeries aménagées par l'endofaune (Bledius, Corophium, Nereis) soit à la texture même des sédiments (sables poreux à Bledius arenarius).

La texture des sols de la haute slikke est très variée, ici ce sont des sables, là des argiles, ailleurs des vases peu humides; mais la teneur en eau et la texture des sols sont associées de telle sorte, que le sédiment se prête bien à l'edification de galeries à parois stables, cohérentes, durables. L'humectation par la marée, ou par une nappe phréatique souterraine, intervient en tant que mécanisme régulateur de l'humidité des sols, et donc de leurs propriétés mécaniques; celà explique à la fois la dispersion des faciès de la haute slikke et leur zonation peu stricte. Ce qui compte pour la faune, ce n'est pas tant la durée des inondations mais davantage l'aptitude des sols à l'aménagement des galeries; les sables poreux à Bledius arenarius semblent échapper à cette règle, mais tant l'estimation indirecte, que l'observation des animaux lors de l'arrivée 
du flot, montrent que ces sables, malgré leur grande perméabilité, ne sont pas complètement inondés à marée haute.

\section{La faune de la hate slikke}

Le slistes de faune et diverses remarques concernant les espèces citées sont données plus loin. Il importe de souligner:

(1) L'importance quantitative de la macrofaune endogée; même les espèces vagiles de surface (insectes, araignées) sont capables, lors de l'inondation, de chercher un abri contre l'immersion dans les galeries creusées par la faune endogée, ou dans des abris aménagés sommairement.

(2) Le caractère tantôt médiolittoral, tantôt supralittoral, de la macrofaune endogée. Nous avons établi la distinction entre faunes médio et supralittoral sur un critère simple, à savoir que les galeries sont ou non inondées lors de la haute mer. Par des exemples et des mesures précises (Coropbium volutator, Bledius spectabilis), nous avons testé cette différence en appréciant le volume en air du sol lors de la haute mer. Il n'est pas douteux que, loin de constituer des exceptions, ces exemples situent au contraire des types d'adaptation fondamentalement différents, dont le degré de généralité dépasse largement les espèces choisies.

(3) La cohabitation à des niveaux identiques et dans des aires proches de ces faunes médio et supralittorale. Cette cohabitation est rendue possible par la texture des sols, qui se prêtent bien à l'édification de galeries permanentes, ouvertes ou non à la pénétration de la marée suivant les exigences écologiques de chaque espèce. La zonation est rétablie dans le cas de sédiment poreux très perméable; par exemple Bleditus arenarius, insecte supralittoral, surmonte les amphipodes médiolittoraux du genre Bathyporeia.

(4) Le caractère strictement halophile de la majeure partie des espèces étudiées d'origine continentale (insectes, myriapodes). Les araignées de la haute slikke toutefois, sont plutôt hygrophiles ou paludicoles que véritablement halophiles. Dans son ensemble, la haute slikke apparaît comme un milieu hautement sélectif, où pullulent un petit nombre d'espèces étroitement adaptées, difficilement colonisé par d'autres espèces même réputées ubiquistes.

De tels caractères soulignent l'originalité de la haute slikke et l'opposent à la fois à la slikke et au schorre. Les espèces de la haute slikke sont rassemblés dans le Tableau 19.

\section{Remerques systématiques et écologiques}

\section{Cnidaires Hexacoralliaires}

Diadumene luciae (VERRIL); petite espèce qui appartient également aux niveaux élevés de la slikke, très abondante par endroit dans la haute slikke, notamment dans les sables vaseux de Claouey, entre les salicornes; constante dans toute la baie dans les sables vaseux à Corophium - Vaucheria, les sables argileux à Bledius spectabilis, les microfalaises; densité très variable. 
Tableau 19

Liste des espèces de la haute slikke

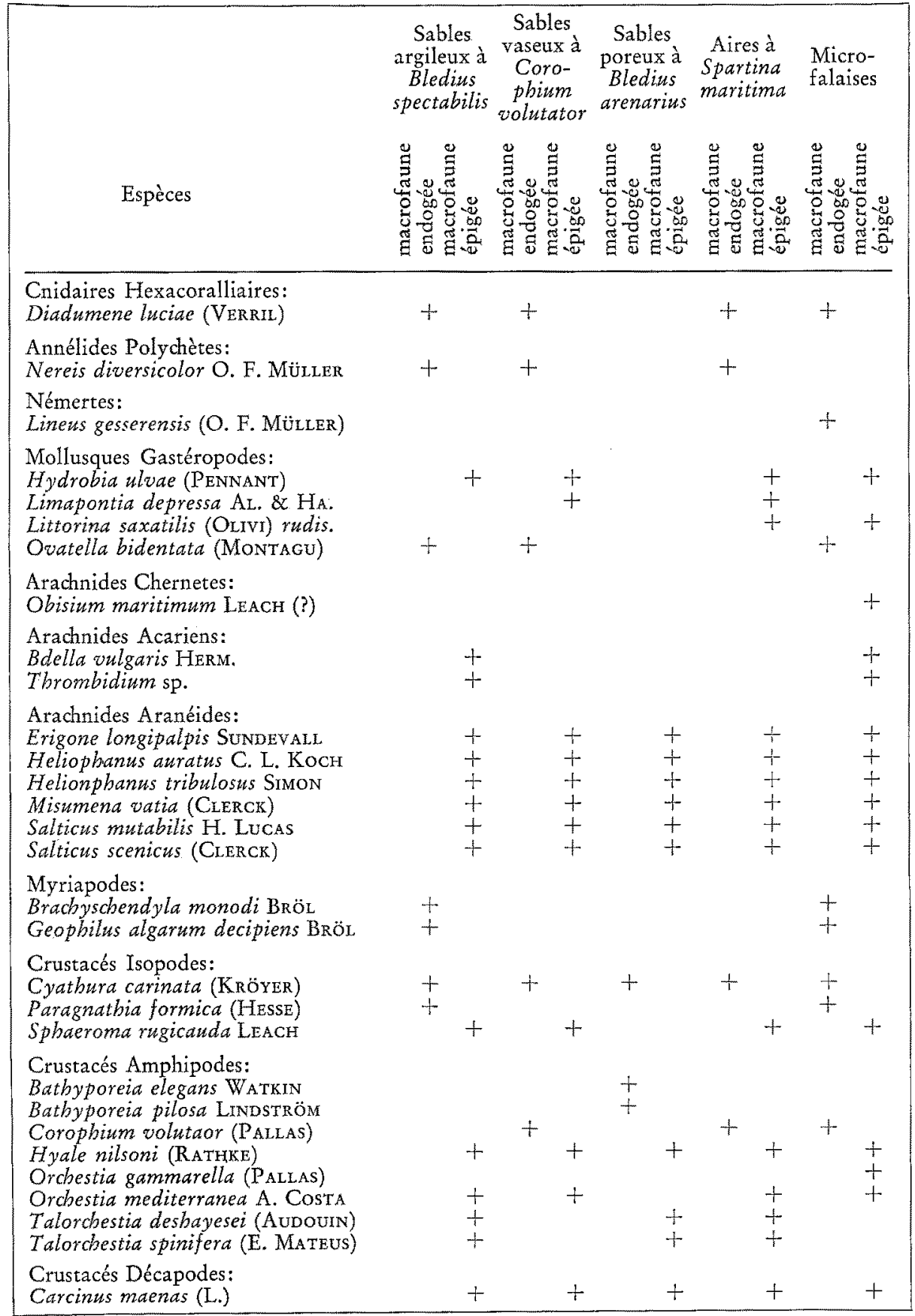


Tableau 19 (suite)

\begin{tabular}{|c|c|c|c|c|c|}
\hline \multirow[b]{2}{*}{ Espèces } & \multicolumn{2}{|c|}{$\begin{array}{cc}\text { Sables } & \text { Sables } \\
\text { argileux à vaseux à } \\
\text { Bledius } & \text { Coro- } \\
\text { spectabilis } & \text { phium } \\
& \text { volutator }\end{array}$} & $\begin{array}{l}\text { Sables } \\
\text { poreux à } \\
\text { Bledius } \\
\text { arenaritus }\end{array}$ & $\begin{array}{l}\text { Aires à } \\
\text { Spartina } \\
\text { maritima }\end{array}$ & $\begin{array}{l}\text { Micro- } \\
\text { falaises }\end{array}$ \\
\hline & 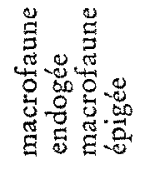 &  &  & 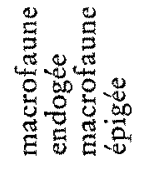 & 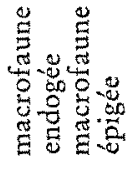 \\
\hline $\begin{array}{l}\text { Insectes Collemboles: } \\
\text { Anurida maritima (GuḱRIN) } \\
\text { Archisotoma pulchella (MONIEZ) } \\
\text { Onychiurus debilis (MoNIEZ) }\end{array}$ & + & + & + & + & $\begin{array}{l}+ \\
+ \\
+\end{array}$ \\
\hline $\begin{array}{l}\text { Insectes Coléoptères: } \\
\text { Bledius arenarius PAYKuLL } \\
\text { Bledius spectabilis KRAATZ } \\
\text { Bledius unicornis GERMAR } \\
\text { Cillenus lateralis SAMOUELLE } \\
\text { Diglossa submarina FARM } \\
\text { Dyschirius salinus SCHAUM } \\
\text { Heterocerus maritimus GUERIN } \\
\text { Heterocerus flexwosus STEPHENS } \\
\text { Pogonus cbalceus MARSHAM } \\
\text { Tachys scutellaris STEPHENS }\end{array}$ & $\begin{array}{l}+ \\
+ \\
+ \\
+ \\
+ \\
+\end{array}$ & $+{ }^{+}$ & $+\begin{array}{r}+ \\
+\end{array}$ & $\begin{array}{l}+ \\
+ \\
+ \\
+\end{array}$ & $\begin{array}{l}+ \\
+ \\
+ \\
+ \\
+ \\
+\end{array}$ \\
\hline
\end{tabular}

Annélides Polychètes

Nereis diversicolor O. F. MúlLER; seul polychète de la slikke susceptible de coloniser de manière dense et régulière les faciès médiolittoraux de la haute slikke; lié alors aux sols-vaseux dont l'humidité est en rapport avec la présence de nappes souterraines peu profondes d'eau douce.

\section{Némertes}

Lineus gesserensis (O. F. Müller); pour Joubrn (1894), la zone «qui n'est pas recouverte tous les jours par la marée, et qui peut, en temps de morte eau, restée à sec un ou deux jours consécutifs ... est caractérisée par une seule espèce de némertes, que l'on y rencontre resque toujours, mais qui descend aussi bien au-dessous: c'est Lineus gesserensis (O. F. MülLER)». Cette espèce a une large répartition et a été signalée notamment à de nombreuses reprises dans les microfalaises, au pied des schorres (MONod 1926). Salmon (1959) signale dans l'Ic Lineus longissimus Sowerby; je rapporte à Lineus du groupe gesserensis les némertes des microfalaises d'Arcachon mais avec un doute sur leur position systématique exacte. 


\section{Mollusques Gastéropodes}

Hydrobia ulvae (Pennant) étagé de la slikke jusqu'au sommet du schorre.

Limapontia depressa A. \& H.; espèce assez commune dans le feutrage des Vaucheria et des Enteromorpha de la haute slikke. CUENOT (1927) signale Limapontia nigra (=L. depressa ?) à Arcachon, dans les algues vertes, de juillet à septembre, et a constaté la ponte en juillet.

Littorina saxatilis (Otrvi) var. rudis Maton; la variété rudis, liée au mode calme, de Littorina saxatilis, est largement répartie dans l'ensemble de la haute slikke, particulièrement dans les aires à spartines où parfois chaque tige porte un ou plusieurs individus. Espèce signalée dans de nombreux estuaires. (Sollaud 1925, Monod 1926, Cazalet 1935, etc.).

Ovatella bidentata (MONTAGU) (= Leuconia bidentata = Alexia bidentata); ce petit Auriculidé a été signalé sous divers noms, dans tous les estuaires de nos côtes mais certaines citations paraissent peu sûres; par exemple Monod (1926) cite Alexia bidentata Mont. dans la Basse Seulle: «Sous les Phragmites que la marée échoue à la surface des Obione... pullulent les Orchestia gammarella... les Sphaeroma rugicauda LEACH, et les Alexia bidentata MONT.» L'habitat et la faune associée sont plutot ceux d'Alexia myosotis (Drap.) qui n'est pas cité; or dans la Basse Seulle j'ai constaté cependant la présence d'Alexia myosotis qui est plus grande, plus abondante, plus facile à récolter qu'Ovatella bidentata (= Alexia bidentata), plus petite, plus abritée, en position plus basse. Si la confusion reste ici incertaine il n'en est pas de même dans le travail de Cazalet (1935), où Leuconia (Alexia) bidentata Mont. et Alexia myo. sotis sont indifféremment cités et incontestablement confondus. SALMoN (1959) cite dans l'estuaire de l'Ic Lenconia (Alexia) bidentata MonT., sur la vase semi-dure avec Paragnatbia formica HESSE, et sous les pierres avec Sphaeroma rugicauda et Orchestia gammarella, mais aussi «au niveau de l'Obionetum et du Puccinellietum» avec Peringia ulvae ( $=$ Hydrobia ulvae): «Leuconia bidentata a une préférence écologique très marquée dans le cas de l'Tc, pour le schorre moyen dans lequel il trouve son optimum de développement»; Alexia myosotis n'est pas cité. Ces exemples pourraient être multipliés. Bien qu'Ovatella bidentata (Montagu) et Alexia myosotis (Drap.) soient deux espèces bien distinctes, qui ne se ressemblent nullement te ne peuvent être confondues, leurs habitats voisins dans les estuaires, la carence des renseignements systématiques les concernant, ont entraîné de nombreuses confusions de la part des non spécialistes, les deux espèces étant prises ou citées l'une pour l'autre; à fortiori, on ne peut donc s'attendre à trouver aisément des précisions écologiques différentielles à leur sujet.

Mars (1959) a bien rétabli la distinction entre les deux espèces, qui sont citées l'une et l'autre de l'étage supralittoral, en bordure des étangs saumâtres méditerranéens, Alexia myosotis étant plus commun qu'Ovatella bidentata. Dans les plages à marée du littoral atlantique et de la Manche les deux espèces, bien que toutes deux supralittorales, sont nettement zonées; tandis qu'Alexia myosotis est lié au schorre tabulaire (voir chapitre suivant), Ovatella bidentata est localisé à la haute slikke, soir dans le front des microfalaises, soir dans la partie superficielle, en général un peu 


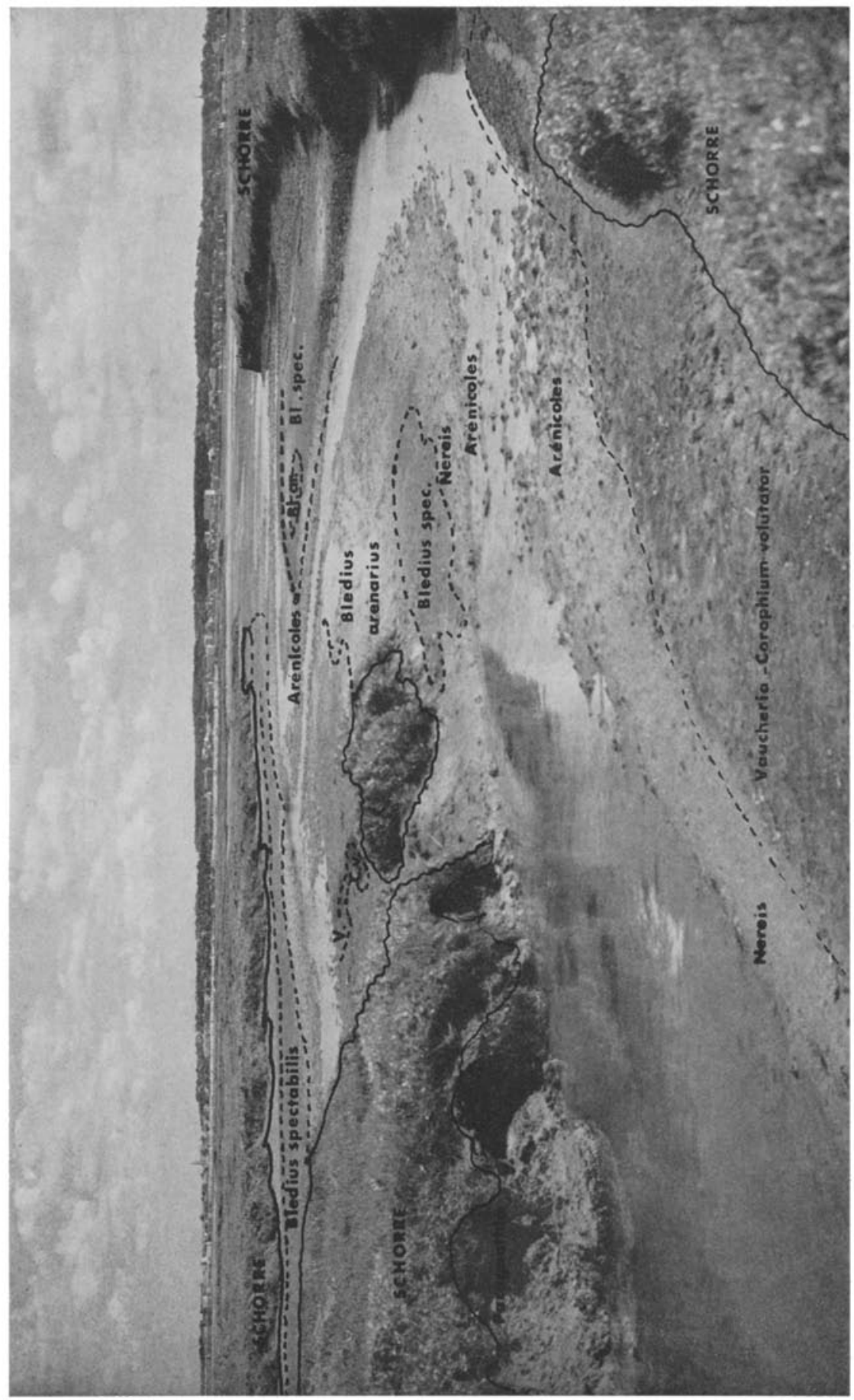

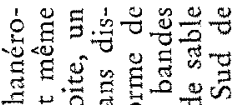

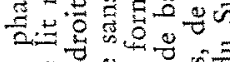

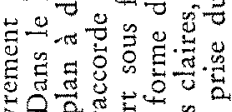

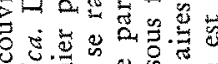

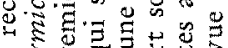

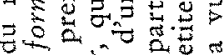

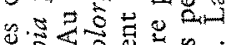

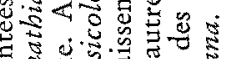

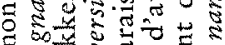
每 है

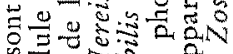

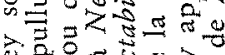

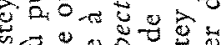

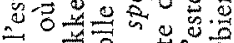
क

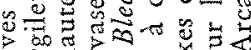
ज包 o

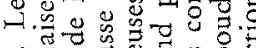

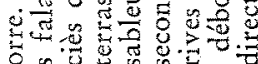

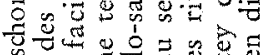
का


है: 웡응

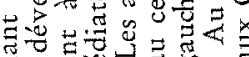

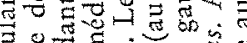
क 3. 궁



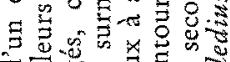

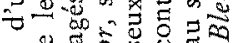

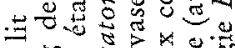
च $50 \%$ ए 03. Jै

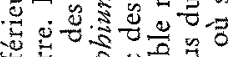


论

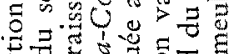


另 ๙

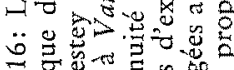

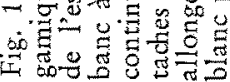


enterrée, des sables vaseux à Corophium ou des sables argileux à Bledius spectabilis (Arcachon, Ile aux Oiseaux). Plus rarement, Ovatella bidentata colonise faiblement le Puccinellietum, s'abritant alors avec Assininea sp. dans les microcavités très humides, amenagées sous les pierres reposant à la surface d'un sol argileux (Gujan-Mestras).

\section{Arachnides Chernetes}

Je rapporte avec doute à Obisium maritimum LEACH un petit chernète récolté, assez rarement d'ailleurs, sur les microfalaises de llle aux Oiseaux.

\section{Arachnides Acariens}

Quelques récoltes, confiées à divers spécialistes, sont en cours de détermination. Bdella vulagirs Herm. (var. littoralis MONIEZ ?) est abondant durant tout l'été dans les crevasses de la microfalaise; divers Thrombidium (sensu lato) sont accidentels.

\section{Arachnides Aranéides}

Selon DenIs (1951): «à l'exception d'Halorates reprobus (O. P. CAmbr.) qui paraît inféodé aux bords de mer, il n'existe pas, sur le littoral de la France occidentale d'araignées spéciales aux terrains salés»; sur le littoral méditerranéen on connaît depuis longtemps l'habitat original de Desisiopsis racovitzai que l'on récolte dans les Lithothamnium.

Les araignées qu l'on récolte sur les plages meubles abritées, sont des formes plutôt hygrophiles ou franchement paludicoles, que des formes véritablement halobies.

Micryphantidae: Erigone longipalpis SUNDEVALI; petite espèce qui colonise abondamment le schorre d'où elle envahit, surtout en été, la haute slikke. Solzaud (1925), la considère comme caractéristique des terrains salés et signale qu' «elle tisse ses toiles délicates à la base des feuilles de Glyceria». BerLAND (1932) cite dans la liste des araignées marines indigènes, Erigone dentipalpis.

Thomisidae: Misumena vatia (CIERCK); il s'agit là d'une espèce banale qui, bien que non caractéristique des prés salés, y est largement représentée. Misumena vatia est très abondante dans le schorre inférieur et la haute slikke, en compagnie notamment de divers salticides; très bien adaptée à son milieu au point de vue chromatique, elle échappe souvent à la capture; en outre, cette espèce chasse à l'affût et, poursuivie, elle ne quitte son abri qu'au dernier moment, son premier réflexe étant de s'immobiliser et non de fuir. Misumena vatia se laisse aisément recouvrir par la marée, dont elle s'abrite sommairement dans les anfractuosités de l'argile ou sous des racines; à marée basse il n'est pas rare de la voir chasser sur la slikke nue, où elle capture en particulier le collembole Anurida maritima.

Salticidae: Salticus mutabilis H. Lucas, Salticus scenicus (Clerck), Heliophanus 
auratus C. L. Koch, Heliophanus tribulosus Srmon; il s'agit là d'espèces banales qui m'ont paru communes et très vagabondes, capables en particulier de descendre à marée basse jusqu'au niveau de la slikke où elles chassent diverses proies.

\section{Myriapodes}

Brachyschendyla monodi Bröl; espèce décrite par Brolemann (1924) d'après une femelle récoltée dans le Finistère; le mâle, resté longtemps inconnu, a été découvert (et décrit) en 1961, par Demange dans mes collections d'Arcachon. L'espèce est cependant commune et a certainement été bien souvent récolté mais confondue sans doute avec Hydroschendyla submarina GRUBE. Brachyschendyla monodi est un halobie étroitement localisé à la microfalaise des plages abritées (Roscoff, estuaire de la Penzé; Chaillevette, estuaire de la Seudre; dans la baie d'Arcachon, sur l'Ile aux Oiseaux et à Gujan Mestras). Au prix de quelque attention, on récolte des échantillons de cette espèce toute l'année, mais c'est surtout en juin et juillet que les chasses sont plus fructueuses. Ponte observée à plusieurs reprises en juin; la ponte est réduite à 4 ou 5 oeufs, sur lesquels la femelle se tient lovée; des oeufs récoltés dans la nature et ramenés au laboratoire sans la femelles évoluent incomplètement; par contre j’ai pu obtenir quelques éclosions en élevant simultanément la ponte et la fémelle qui la protège. Il est difficile de contrôler le comportement de cette femelle pendant l'incubation, en effet, si on la dérange trop fréquemment ou que l'on découvre la logette, elle abandonne la ponte définitivement. Dans la nature, j’ai récolté des jeunes nouveaux-nés à partir de juillet.

Geopbilus algarum decipiens BRöL; halobie de la banquette inférieure du schorre, où il cohabite avec Bracbyschendyla monodi mais moins abondant que ce dernier. Selon Brolemann (1932) signalé de la Manche, de la Loire inférieure, de la Charente inférieure. SALMON (1959) l'a récolté également dans la microfalaise du schorre de l'Ic; plus en amont, il est remplacé dans le même faciès et au mêne niveau par Scolioplanes maritimus LEACH.

Je n'ai jamais récolté à Arcachon Hydroschendyla submarina Grube, espèce citée par de nombreux auteurs, peut être abusivement, dans des sites analogues à ceux que j'explorais.

\section{Crustacées Isopodes}

Cyatbura carinata (KRöYER); espèce à large répartition, déjà signalée dans la slikke et qui culmine dans la haute slikke.

Paragnathia formica (HEsse); c'est incontestablement l'une des espèces les plus caractéristiques de la haute slikke, dont elle occupe tous les faciès sablo-argileux et particulièrement le front des microfalaises. Les adultes aménagent des nids dans lesquels les femelles s'abritent, en compagnie des mâles, pendant la gestation (voir Fig. 15). J'ai donné antérieurement des précisions sur le cycle de cette espèce à Arcachon (AMANIEU 1963). 
Sphaeroma rugicauda LEACH; espèce bien connue pour habiter les plages saumâtres abritées, liée surtout aux flaques supralittorales et aux abris humides du schorre, mais, très mobile, s'étend parfois jusque sur la slikke. Dans la haute slikke, Sphaeroma rugicauda occupe surtout le sommet des microfalaises, comme l'a signalé et figuré MoNoD (1926).

\section{Crustacés Amphipodes}

Batbyporeia elegans WATKIN; «it is in general a shallow-water form» (WATKIN 1938) infra ou médiolittorale, citée soit des régions marines, soir des régions plus ou moins saumâtres d'estuaires (Leloup \& KonIETZKo 1956, VADER 1965).

Bathyporeia pilosa LINDSTröm; colonise les sables saumâtres intertidaux (voir par exemple les stations citées par WatKin 1938). Salvat (1962) rapporte que, sur les plages meubles semi-abritées d'Arcachon, cette espèce «colonise les sédiments jusqu'au niveau $+3,18 \mathrm{~m}$... néammoins les densités maxima ... se situent dans la partie inférieure de l'étage mésolittoral». C'est également celui des Haustoriidés étudiés par VADER (1965), dont le maximum de densité se situe dans la position la plus élevée; les histogrammes publiés par cet auteur conduisent à admettre également une zonation assez stricte. VADER (1965) précise en outre en réponse à une question de SEGERSTRALE que «The lowest chlorinity at which $B$. pilosa has been found within the Deltaic region was about $4 \%$, corresponding to a salinity of about $7 \%$. But in the EemsDollart estuary, in the North Eastern part of country, the species has been collected at chloronities down to $1,5 \%$ (STOCK \& DE VOS 1960).» Les chiffres donnés par VADER sont voisins de ceux que $j$ 'ai notés dans la région de Lège ( $\mathrm{O} \%$ compris entre 6,4 et 10,3, à marée basse, en diverses stations, février 1962). Les populations de Bathyporeia pilosa à Lège, ont leur maximum de densité au-dessus de 3 mètres, c'est à dire que ce maximum correspond à la limite supérieure de la partie de l'estran colonisée à la Vigne; mais la très faible pente de la plage de Lège et la proximité du lit de la rivière, entretiennent une humidité permanente et permettent d'estimer qu'à Lège, comme à la Vigne, Bathyporeia pilosa occupe, du point de vue de l'hydro dynamisme interstitiel, des positions équivalentes.

Hyale nilssoni (RATHKE); sous les débris humides, épaves, laisses, Fucus couchés.

Orcbestia gammarella (PALLAs); espèce du schorre qui s'avance dans la haute slikke durant les mortes eaux estivales; lors du revif, Orchestia gammarella reflue vers le schorre avec le front de la marée. Quignard (1963), a noté, en bordure des étangs méditerranéens, des migrations de diffusion de divers Orchestia; en hiver les Orchestia se groupent sous des abris variés et notamment sous les laisses de zostères qui offrent une protection thermique; «à mesure que la chaleur et la sécheresse s'accentuent ces crustacés quittent le haut rivage pour se rapprocher de l'eau». A Arcachon, de telles migrations sont moins nettes, ce qui tient sans doute au caractère moins extrême des conditions climatiques.

Orchestia mediterranea A. Costa; espèce préférentiellement liée à la haute slikke, accidentelle dans le schorre inférieur. Répartition et cycle à Arcachon étudiés dans le travail de Amanieu (1969). 
Talorchestia deshayesei (Audoun); espèce supralittoral liée aux sables secs recouverts de laisses peu épaisses, également desséchées. Large répartition dans le schorre, accidentelle dans la haute slikke. Présence très irrégulière, bien qu'en général elle pullule lorsqu'elle est présente; les stations repérées sont extrêmement instables, apparaissant ou disparaissant à quelques jours d'intervalle. Plages abritées et semi-abritées.

Talorcbestia spinifera (E. Mateus); stations assez semblables à celles de l'espèce précédente mais ne pullule jamais à Arcachon, qui se situe peut être au voisinage de la limite nord de son aire géographique de répartition, alors qu'elle est beaucoup plus abondante au sud, notamment en Espagne et au Maroc (communication personnelle de M. Ex Kam). Amanieu \& Salvat (1963 \& 1965) ont donné des précisions sur la systématique et la répartition de cette espèce, décrite en 1962 du Portugal par Mateus, mais qui a été récoltée au moins dès 1910 par Chevreux (confondue alors avec Orchestoïdea fischeri).

\section{Insectes Collemboles}

Anurida maritima (GuérIN); une des espèces les plus caractéristiques de la haute slikke. Lors du recouvrement par la marée, des populations entières se laissent flotter et sont ainsi transportées jusque sur le schorre. Mais les populations les plus stables et les plus denses sont installées sur les argiles ou les vases de la haute slikke, notamment dans les anfractuosités de la microfalaise, où sont déposées leurs pontes de couleur jaune orangé, ainsi que leurs exuvies blanches; cet habitat est assez semblable «aux fentes des faciès rocheux» qui, selon Delamare-Debouttevilie (1950), constituent thabitat préférentiel d'Anurida maritima.

Archisotomo pulchella (Moniez); espèce du Puccinellietum, accidentelle dans la haute slikke. Toutefois Sollaud (1925) \& MoNod (1926) citent Archisotoma besselsi (PAckard) des slikkes à Nereis de la Slack et de la Basse Seulle; leurs récoltes avaient été examinées par Denis, qui concluait à la synonymie entre Archisotoma pulchella et $A$. besselsi (voir DenIs 1924). La distinction entre les deux espèces a été rétablie par Strenzie (1955); Delamare-Deboutteville (1950) précise qu'Arcbisotoma besselsi «vit à l'intérieur du sable au niveau des infiltrations d'eau de mer».

Onychiurus debilis (Moniez); assez dense dans des stations très localisées. Espèce strictement marine selon Delamare-Debout'TEville (1953).

\section{Insectes Diptères}

Diverses larves trouvées ne sont pas déterminées.

\section{Insectes Coléoptères}

Bledius spectabilis KRAATZ, Bledius unicornis Germar, Bledius arenarius PAYKULL; les trois espèces sont des halobies étroitement liés aux sols salés, saumâtres ou marins. Cillenus lateralis SAmouelle; est sans aucun doute avec Bledius spectabilis, le coléoptère le plus abondant et le plus caractéristique de la haute slikke. Selon Temperz: 
(1934): «Cillenus lateralis SAM. fréquente les rivages où le sable est fin et mélangé de plus ou moins de limon, dans la zone recouverte à marée haute. On le trouve même dans des stations franchement vaseuses. Au banc de Pineau, (dans la baie d'Arcachon) où je l'ai observé en particulière abondance, en août 1917, un quart seulement des individus se rapportait à la forme typique, par leur prothorax franchement vert; un peu plus d'un quart ayant ce segment testacé et sans reflet métallique, appartenaient à la variété bedeli NIC. le reste était constitué par des spécimens intermédiaires, à corselet testacé, avec un reflet vert métallique plus ou moins accentué.» En élevage, Cillenus lateralis creuse très activement le sable; dans la nature, il s'enterre au moment de la haute mer qu'il ne fuit pas; très actif surtout la nuit, où, au début de l'été, on peut en recenser plus d'une centaine par mètre carré sur les rives de l'Ile aux Oiseaux. Accouplements observés en juin et début juillet, époque à laquelle plus de 50\% des individus captutés sont pris in copula. BAUdorn (1964) cite Cillenus lateralis dans la faune des sables bulleux, avec notamment Diglossa mersa.

Diglossa submarina Farrm.; est une petite espèce qui échappe assez facilement au tri; lorsqu'on la recherche avec précaution, il est rare de ne pas la trouver dans les mêmes biotopes que les Bledius.

Dyscbirius salinus Schaum; est un petit carabique halobie, lié à la haute slikke, où il cohabite avec les Bledius; on a signalé (voir Burmerster 1939), des associations entre Dyschirius et Bledius, les premiers étant inféodés aux seconds en tant que prédateurs; JEANNEL (1941) rappelle cependant que, selon les observations de DE Brunier (1931) «il n'est pas confirmé que le Dyschirius soit parasite du Bledius; il l'accompagne toutefois, même au cours de ses migrations». Dans la nature, Dyschirius salinus m'a paru être un entomophage assez éclectique; en élevage, il accepte des proies variées, notamment des collemboles (Anurida).

Heterocerus (Littorimus) maritimus GuérIN; est un halobie régulièrement présent sur la banquette inférieure des schorres, ou au niveau de contact entre schorres et slikkes, les imagos creusent leurs galeries dans les sols sablo-argileux compacts; les pontes, composées d'une vingtaine d'oeufs, sont déposées au début de l'été (juin), au fond d'une galerie rectiligne aveugle, terminée par une petite chambre. Au laboratoire, on peut observer que les imagos surveillent la ponte au moins pendant plusieurs jours, sans doute jusqu'à l'éclosion (que je n'ai pu obtenir en élevage). Présence des imagos notée presque toute l'année, mais ils ne sont abondants qu'en mai et juin, alors qu'en hiver les captures sont accidentelles; des larves âgées, récoltées dans la nature en mai, ont donné des imagos début juin au laboratoire.

Heterocerus flexnosus STEPHENs; est également un halobie mais plus rare que le précédent avec lequel il cohabite; présence notée surtout en été, de juin à août.

Pogonus chalceus Marsham; est un halobie du schorre qui descend accidentellement sur la haute slikke à marée basse, surtout de nuit, en incursion nutritielle.

Tachys scutellaris STEPHENs; est un halobie abondant dans la haute slikke avec les espèces précédemment citées; il vit dans les anfractuosités du sol dont on le fait sortir en piétinant. 


\section{LE SCHORRE ET LA ZONE PARHALIENNE}

Au dessus de la slikke ou de la haute slikke débute le schorre proprement dit, caractérisé à la fois par son recouvrement phanérogamique dense et continu et par la rareté des inondations qui l'atteignent. Deux associations phanérogamiques essentielles sont susceptibles d'occuper le schorre, d'une part le Puccinellietum maritimae, d'autre part le Juncetum maritimi.

Le Puccinellietum maritimae occupe les zones halophiles des schorres littoraux. Il est limité à sa base par les faciès référables à la haute slikke, mais vient parfois directement au contact de la slikke proprement dite; au sommet, sa marge continentale est le plus souvent marquée par une ceinture d'Agropyrum, qui culmine au contact de la zone parhalienne.

Le Juncetum maritimi est lié, dans la baie d'Arcachon, aux prairies hygrophiles saumâtres; il prend sa pleine extension dans la région de Lège et Arès. A sa base, il vient généralement au contact direct de la slikke. A son sommet il culmine, comme le Puccinellietum, au contact de la zone parhalienne. Une partie importante du Juncetum est colonisée par la faune médio-littorale (Nereis diversicolor, Corophium volutator, Bathyporeia) de la haute slikke, qu'il est difficile de délimiter géographiquement, de telle sorte que le Juncetum s'étend en pratique, depuis les niveaux adlittoraux non inondables, jusqu'au contact de la slikke infra-littorale.

Quant à la zone parhalienne, elle occupe les niveaux qui ne sont pas inondables de manière systematique mais peuvent être recouverts par la marée une dizaine de fois chaque année, en hiver, à l'occasion de circonstances exceptionnelles (marées de très fort coefficient, coïncidant avec une période de tempête). Elle abrite une flore en partie halophile mais aussi une faune vagile essentiellement haloxène. Elle sert également de refuge, lors des fortes marées, à la faune vagile supra-littorale du schorre qui vient s'y abriter de l'immersion.

Les Agropyrum constituant un bon repère permettant de séparer le schorre de la zone parhalienne, il était intéressant de préciser leur répartition hypsométrique autour de la baie d'Arcachon.

\section{Répartition hypsométrique des Agropyrum autour de la baie d'Arcachon}

La Teste: A l'angle sud-est du "pré salé» de la Teste, les Agropyrum s'installent au sommet d'une petite plage de sable; le peuplement débute à la cote $+4,32 \mathrm{~m}$; Salsola soda L. descend un peu plus bas que les graminées.

La Hume: La butte sur laquelle est installé le repère géodésique de Gujan Mestras V, est ceinturée par une bande d'Agropyrum dont la base est à $+4,51 \mathrm{~m}$. Salsola soda croît légèrement plus haut (base à $+4,54 \mathrm{~m}$ ); viennent ensuite Hordeum maritimum WITH., qui débute à $+4,56 \mathrm{~m}$, Beta maritima L. et Atriplex bastatus L. à $+4,72 \mathrm{~m}$; en revanche Inula critbmoïdes L. apparaît par touffes au-dessous de $+4,00 \mathrm{~m}$. 
Mestras: Les Agropyrum débutent à la cote $+4,04 \mathrm{~m}$, sur les bords d'un talus de sable bien égoutté qui culmine à $+4,36 \mathrm{~m}$. Sur un autre talus qui domine directement la haute slikke, on retrouve un peuplement d'Agropyrum à partir de $+4,38 \mathrm{~m}$ en compagnie de Spergularia marginata KITTEL et d'Obione portulacoïdes.

Gujan: En haut du Puccinellietum de Gujan, les Agropyrum font suite aux halophytes et principalement à Glyceria maritima (Huds.) WaHLg. à partir de la cote $+4,17 \mathrm{~m}$.

Le Teich: Les Agropyrum sont disposés le long des digues des réservoirs, à partir de la cote $+4,34 \mathrm{~m}$; Salsola soda débute plus haut à $+4,76 \mathrm{~m}$ et Beta maritima à $+5,11 \mathrm{~m}$.

Lanton: J'ai noté un peuplement d'Agropyrum repens (L.) P.B. à l'extrémité nord de la digue du port de Lanton; la base de ce peuplement débute à $+4,41 \mathrm{~m}$, en même temps que les premiers pieds de Beta maritima et de Salsola soda.

Arès: Au sommet du Juncetum d'Arès, les Agropyrum apparaissent à $+4,12 \mathrm{~m}$ en même temps que les premiers Baccharis halimifolia L. Non loin du courant de Lège, les Agropyrum débutent dès $+4,05 \mathrm{~m}$, en compagnie de Juncus maritimus, Plantago maritima L., Atriplex littoralis L., Suaeda maritima (L.) DumorT., Obione portulacoides etc.; ici le substratum du schorre, qui vient mourir au pied de la dune, est particulièrement sableux et perméable.

Lugue du Cap-Ferret: Autour de la lugue du Cap-Ferret, Agropyrum junceum (L.) P.B. apparaît à partir de $+4,32 \mathrm{~m}$; non loin de là, quelques pieds d'Honckenya peploides (L.) EHrH. débutent à $+4,02 \mathrm{~m}$ en même temps que les Atriplex; Artemisia campestris L. et Helichrysum staechas (L.) DC. apparaissent au-dessus $\mathrm{de}+4,90 \mathrm{~m}$.

Ile aux Oiseaux: Autour de la butte du «Truc vert», la ceinture des Agropyrum apparâ̂t à $+4,21 \mathrm{~m}$ soit un peu au dessus de Suaeda fruticosa (L.) Forsk. $(+4,12 \mathrm{~m})$ et au dessous de Baccharis halimifolia $(+4,54 \mathrm{~m})$. Sur les plages nord, les Agropyrum apparaissent à $+4,07 \mathrm{~m}$, en même temps que divers Atriplex et Salsola soda en des sites où la pente de la plage est assez forte et le substratum très sableux; Baccharis halimifolia, Carex arenaria L., et même Erica scoparia L. apparaissent sur les mêmes plages à la cote $+4,70 \mathrm{~m}$.

Plages Oceaniques: A titre de comparaison je signale que, selon Caussanei. (1962) la plage herbeuse, en bordure du littoral océanique au sud d'Arcachon, débute seulement $\grave{a}+6,00 \mathrm{~m}$ environ, soit très sensiblement au dessus des niveaux qui ont été notés en bordure des plages abritées.

Ces mesures font ressortir que les peuplements d'Agropyrum, assez bien zonés autour de l'ensemble de la baie, débutent dans le schorre, alors qu'ils culminent dans la zone parhalienne; le niveau de plus forte densité marque la séparation entre l'un et l'autre.

Niveau de séparation entre schorre et zone parhalienne

D’après des considérations purement marégraphiques, nous avons signalé (AMANIEU 1966) que le niveau $+4,60 \mathrm{~m}$ marquait la limite inférieure de la zone non inondable 


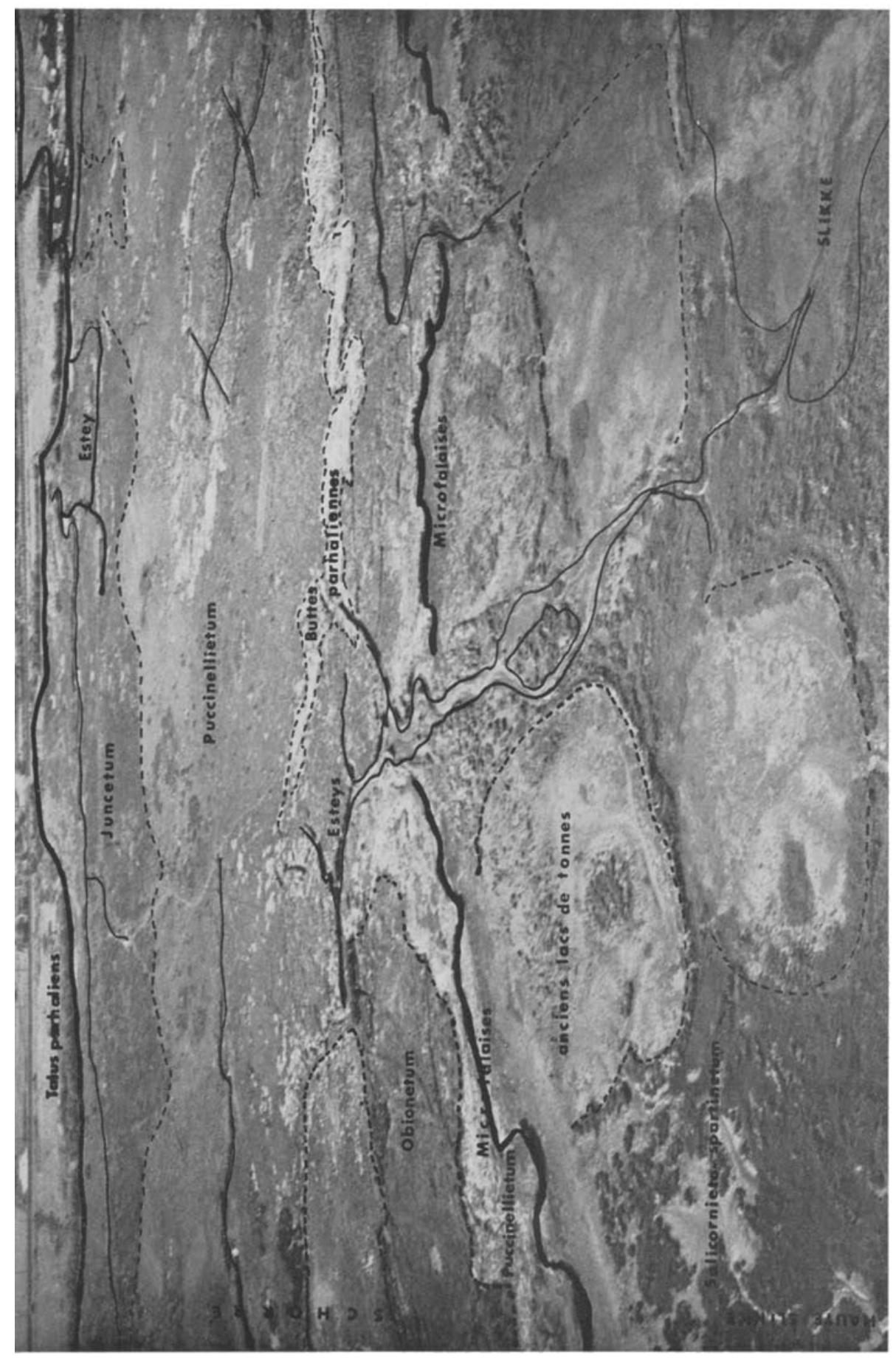



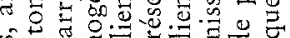

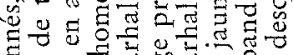


氜. 过 告 o:



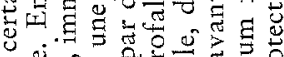
ง.

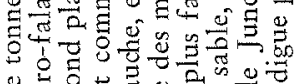

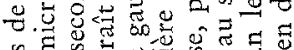

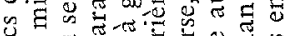


थे

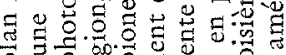

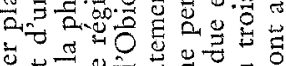

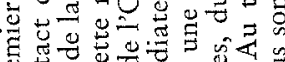



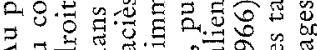
4 Tे 市我 m. है क्षे

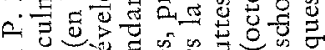

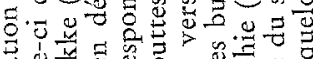

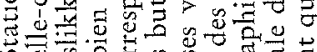

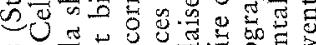



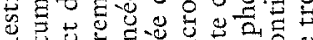

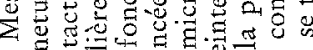
y. o 0.0.

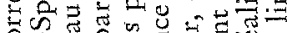

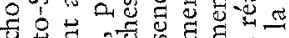



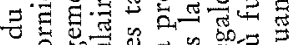

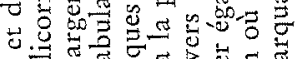

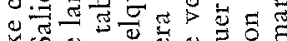


o 光的击

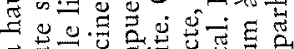
글



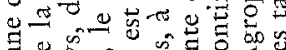

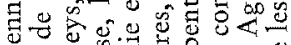

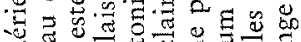





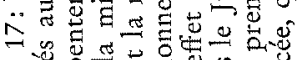

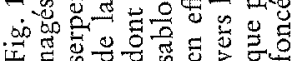


durant les 6 mois d'été, d'avril à septembre. En fait, durant cette période, les conditions marégraphiques varient peu entre $+4,60 \mathrm{~m}$ et $+4,40 \mathrm{~m}$ (voir AMANIEU 1966). Les observations bionomiques s'accordent avec les données marégraphiques, pour fixer à ce niveau la limite entre schorre et zone parhalienne. Il faut se garder toutefois de fixer des valeurs trop absolues, particulièrement en ce qui concerne la faune; nous verrons en effet que, du point de vue faunistique, schorre supérieur et zone parhalienne sont des zones d'échanges importantes entre les espèces qui les peuplent et qui sont presque toujours vagiles. Suivant les marées, suivant les saisons, le stock faunistique se déplace de telle sorte qu'il serait illusoire de rechercher des frontières hypsométriques absolues. En ce qui concerne la flore, les limites sont sans doute plus stables; là encore toutefois, si le niveau $+4,60 \mathrm{~m}$ représente une valeur moyenne raisonnable, il est nécessaire d'admettre certaines fluctuations en rapport avec la pédologie. L'intérêt des nivellements effectués est de permettre une appréciation chiffrée raisonnable de l'amplitude de ces fluctuations.

Les prospections entreprises dans la baie d'Arcachon, portent essentiellement sur le Puccinellietum; des observations plus fragmentaires, relatives au Juncetum et à la zone parhalienne, seront données à titre comparatif.

\section{Les conditions édaphiques. Texture des sols}

La texture des sols référables au Puccinellietum ou à ses variantes et faciès est extrêmement variable; voici quelques chiffres concernant Mestras et l'lle aux Oiseaux; les échantillonnages datés des 2 et 3 septembre 1965 ont été effectués à marée basse, en période de morte eau (Tab. 20).

L'échantillonnage est insuffisant pour que l'on puisse en tirer des conclusions gé-

Tableau 20

Texture et teneur en eau des sols du schorre à Arcachon

\begin{tabular}{|lcccc|}
\hline \multicolumn{1}{|c}{ Stations } & $\begin{array}{c}\text { Particules } \\
\text { fines }(\% \%) \\
<50 \mu\end{array}$ & $\begin{array}{c}\text { Sables }(\%) \\
>50 \mu\end{array}$ & $\begin{array}{c}\text { Teneur en } \\
\text { eau }(\%)\end{array}$ & $\begin{array}{c}\text { Perte de } \\
\text { Poids } \\
\text { par calcina- } \\
\text { tion au four } \\
(\% / 0)\end{array}$ \\
\hline $\begin{array}{l}\text { Ile aux Oiseaux, Truc vert, } \\
\text { sommet du Puccinellietum }\end{array}$ & 13,9 & 86,1 & 33,3 & 6,7 \\
$\begin{array}{l}\text { Mestras, contre la digue, } \\
\text { sommet du Puccinellietum }\end{array}$ & 83,4 & 17,6 & 75,5 & - \\
$\begin{array}{l}\text { Ile aux Oiseaux, Truc vert, } \\
\text { Agropyretum }\end{array}$ & 47,3 & 52,4 & 23,0 & 17,0 \\
Mestras, Agropyretum & 50,6 & 49,4 & 56,2 & 11,8 \\
Ile aux Oiseaux, Obionetum & 100,0 & 0,0 & 156,4 & - \\
Mestras, Obionetum & 77,4 & 22,6 & 100,0 & 11,5 \\
Ile aux Oiseaux, Plage nord, & 4,5 & 95,5 & 11,0 & 2,5 \\
Frankenio-Staticetum & & & & \\
\hline
\end{tabular}


nérales; il permet cependant de montrer la variété des sols du Puccinellietum sensu lato, qui occupe aussi bien des sols exclusivement argileux (Obionetum de l'Ile aux Oiseaux) durs, compacts, imperméables, que des sols sableux très meubles (Frankenio Staticetum). Le contrôle des facteurs édaphiques est important pour les phytosociologues (voir LEMEE 1952, TunMEL 1949); il présente beaucoup moins d'intérêt pour l'étude de la répartition de la macrofaune. Ce qui est le plus remarquable, en effet, dans le schorre et particulièrement dans le Puccinellietum supérieur, c'est l'absence de macrofaune endogée, caractère qui contraste fondamentalement avec la slikke ou la haute slikke; les peuplements zoologiques sont constitués par une épifaune vagile, pourvue de larges moyens de locomotions, qui utilise le sol soit pour se déplacer, soir pour en explorer les abris superficiels. Or, la surface du Puccinellietum est riche en refuges et abris variés; ce sont d'une part les fentes de retrait dûes à la dessication des sols, d'autre part les petites cavités aménagées sous les laisses ou les épaves qui jonchent abondamment le schorre.

Nous aborderons donc immédiatement l'examen des espèces récoltées, en précisant pour chacune l'habitat préférentiel. La distinction entre espèces liées au schorre et espèces liées à la zone parhalienne, doit être tempérée des remarques que nous avons faites concernant les échanges faunistiques entre ces deux types d'habitat, et dont nous donnerons des exemples.

\section{La faune du Puccinellietum de la baie d'Arcachon}

\section{Elements de comparaison avec le Juncetum et la zone parbalienne}

\section{Annélides Oligochètes}

Encbytraeus albidus HENLÉ; quelques oligochètes, particulièrement Enchyiraeus albidus, sont récoltés à la surface du schorre, surtout dans l'humus protégé par les laisses épaisses de zostères et de joncs.

\section{Mollusques Prosobranches}

Assiminea sp; selon Germain (1931), Bavay aurait signalé Assemania ostionum BAVAY «commun à l'embouchure des ruisseaux se jetant dans la partie nord du bassin d'Arcachon entre Taussat et Arès, notamment à Andernos». Je n'ai pas retrouvé cette espèce dans les sites signalés par BAvAY, où pullule actuellement Potamopyrgus jenkinsi; par contre dans le schorre supérieur, sous les hautes laisses échoués à la surface du Pucccinellietum, parfois également (surtout à Mestras) sous les pierres échouées j’ai recolté un Assiminea sp.; selon MAUry, qui a bien voulu examiner mes échantillons d'Arcachon ainsi que ceux récoltés à Talmont (en Gironde), il pourrait s'agir de Assiminea eliae PALAD. Le genre est commun parmi les éléments supralittoraux des faunes des plages abritées.

Hydrobia ulvae (PENNANT); espèce déjà signalée dans la slikke et la haute slikke et qui appartient également à la faune des schorres, Puccinellietum et Juncetum. 
Potamopyrgus jenkinsi (E. A. SMITH); espèce liée aux eaux oligohalines, notamment en bordure des étangs saumâtres d'Arcachon (voir Amanieu 1967). Toutefois Potamopyrgus jenkinsi s'étend aussi dans une partie du Juncetum de Lège, en bordure de la rivière. Les populations observées, très denses d'ailleurs, proviennent du regroupement, sur les vases humides, d'individus entraînés hors du lit du fleuve, au moment des hautes mers de vive eau.

Truncatella subcylindrica (L.); lié, comme Alexia myosotis, au schorre tabulaire mais, contrairement à celui-ci, Truncatella subcylindrica a un comportement très sédentaire et se cantonne sous des abris variés, gravats, débris, laisses, qui ménagent entre eux et la surface du sol des micro-grottes à climat humide; les rassemblements que l'on observe, parfois plusieurs dizaines dindividus dans un même abri, semblent en relation avec la recherche d'un optimum hygrométrique. Selon MARs (1961) les gites de l'espèce seraient beaucoup plus diffus sur les plages méditerranéennes.

\section{Mollusques Pulmonés}

Alexia myosotis (Drap.); l'un des éléments faunistiques les plus constants et les plus caractéristiques de l'ensemble des schorres. Contrairement à Ovatella bidentata avec lequel il a été confondu (voir p. 514) Alexia myosotis est strictement localisé à Arcachon au schorre, mais n'envahit pas la haute slikke. Les pontes sont déposées au début de l'été, principalement en juillet et les éclosions ont lieu début août; en élevage, à la température extérieure, les jeunes grandissent jusqu'à fin novembre puis hivernent; la croissance reprend au printemps et les plus précoces des jeunes nés l'année passée, déposent leurs premières pontes durant l'année en cours, alors qu'ils ont encore une morphologie juvénile correspondant à celle de Pbytia ciliata Morelet (coquille ventrue, présentant près des sutures une frange des poils hérissés); la croissance s'achève durant l'été, une partie des adultes hivernent et déposent de nouvelles pontes alors qu'ils sont eux mêmes âgés de deux ans. La vérification de ce cycle, observé en élevage, est actuellement en cours sur des populations sauvages.

Cochlicella acuta (Müller), Helicella (Candidula) intersecta (POIRE'T), Euparypha pisana (MüLLER); ces trois espèces appartiennent à la zone parhalienne, en bordure des schorres. Les deux premières sont surtout localisées aux biotopes rudéraux arides, particulièrement le long des digues qui marquent la limite continentale du Puccinellietum (Ile aux Oiseaux et littoral sud de la baie diArcachon entre la Teste et le Teich). Euparypha pisana, espèce de la dune herbue (voir SACCHI 1954) est commune dans l'Ammophiletum du littoral landais, beaucoup plus rare en station abritée; elle cohabite occasionnellement avec les précédentes sur les plages nord de lle aux Oiseaux.

\section{Arachnides Aranéides}

Erigonidae: Les Erigonidae sont certainement nombreux dans les prés salés et susceptibles de descendre jusqu'au voisinage de la slikke, mais il s'agit d'espèces de 
petites tailles que je n'ai pas particulièrement recherchées; mes captures ne donnent. donc qu'une idée très imparfaite de leur représentation.

Erigone longipalpis Sundevall; déjà signalé dans la haute slikke; colonise plus abondamment le Puccinellietum.

Gnathonarium dentatum (VIDER); Puccinellietum de l'Ile aux Oiseaux et de Mestras, Juncetum de l'lle aux Oiseaux et de Lège. On capture cette espèce dans les terreaux du schorre, ou plus fréquemment sous les laisses de Juncus des plages calmes du fond de la baie. Adultes à partir de septembre.

Linyphiidae: Lepthyphantes sp., Lepthyphantes tenuis; abondants sous les laisses de Juncus particulièrement dans la région de Lège. Pullulent en été (adultes et juvéniles) sur les digues des claires de Chaillevette (Charente maritime).

Tetragnathidae: Pachygnatha clercki Sündevall, Pachygnatha de geeri Sündevall; espèces banales mais communes dans les près salés; je les ai récoltées tant à Arcachon (surtout $P$. clercki) qu'à Chaillevette, dans l'estuaire de la Gironde et à Hossegor. Signalées dans divers estuaires (MoNod 1926, Basse Seulle, Leloup \& Konietzko 1956, Bas Escaut). La toile réduite à quelques fils, est habituellement tendue parmi les phanérogames dressées, dont le pied est atteint par la marée. Adultes à partir de la fin août; des jeunes, rapportés avec doute à l'une ou l'autre de ces espèces, ont été récoltés au printemps et au début de l'été; subadultes des deux sexes à partir de fin juin.

Lycosidae: Pardosa amentata (Clerck), Pardosa arenicola (CAmbridge); les Pardosa comptent parmi les araignées champêtres les plus communes de notre faune. Les deux espèces citées sont particulièrement abondantes dans le Puccinellietum (Mestras, l'lle aux Oiseaux) beaucoup moins communes dans le Juncetum; on les récolte dans le schorre supérieur, sous les épaves échouées à la surface de l'Obionetum tabulaire. C'est durant le mois de mai, que l'on capture les grosses femelles (surtout $P$. amentata), courant sur le schorre en portant leur cocon; mais en observe également qu'elles aménagent de petite abris entre les épaves et le sol humide; souvent l'on compte cinq à six femelles gestantes, installées côte à côte sous la même épave. Outre la baie d'Arcachon, j'ai récolté également des Pardosa dans les estuaires de la Sladk (Ambleteuse), de la Seine, de la Loire, de la Seudre, de la Gironde, au lac d'Hossegor. Le genre est constamment cité par les auteurs qui ont étudié des régions analogues (MoNoD, JACQUET, LeLoup \& KoniETzKo 1956, etc.).

Tricassa (= Arctosa) subterranea L. Koch; cette intéressante espèce à été trouvée à deux reprises, en mai 1962 et mai 1963, sur le Puccinellietum de l'Ile aux Oiseaux; il s'agissait alors d'adultes des deux sexes. KNÜLLE (1959) la cite des prés salés à Salicornes de Majorque et de Pise.

Arctosa sp.; des stades juvéniles de divers Arctosa, non déterminables spécifiquement, sont capturés sur les Puccinellietum et les Juncetum de la Baie d'Arcachon en même temps que les Pardosa.

Clubionidae: Clubiona (Euryclubiona) stagnatilis Kulczynski, Clubiona (Epiclubiona) neglecta Cambridge; ces deux espèces, particulièrement la première, sont abondantes sous les débris variés (laisses, détritus) échoués sur les Puccinellietum ou les Juncetum. 
Les adultes sont capturés durant tout l'été, et des jeunes, non déterminés spécifiquement, surtout en automne.

Thomisidae et Salticidae: Les espèces appartenant à ces deux familles, déjà citées dans la haute slikke, sont également abondantes dans le Puccinellietum.

Pbilodromus fallax Sundevall; espèce commune dans les dunes du Cap-Ferret; accidentelle dans le Juncetum de Lège.

Divers Tmarus sp. (? stellio E. Srmon); juvéniles ou subadultes, sont assez communs dans le Puccinellietum et s'avancent même jusqu'au niveau des microfalaises.

Les espèces suivantes sont localisées dans la zone parhalienne.

Amaurobiidae: Ciniflo (= Amaurobius) ferox (Walckenaer), Ciniflo (= Amaurobius) erberi (Keyserling); les adultes de ces deux espèces sont abondants dans la zone parhalienne de mars à juin; comme la plupart des espèces appartenant à cette famille, ils tissent leur toile dans les micro-cavités aménagées sous des débris variés, tuiles, planches etc....; communs autours de l'lle aux Oiseaux, puis entre la Teste et Lanton. Au nord, dans la région de Lège Arès, ces mêmes espèces semblent manquer par suite sans doute de la disparition de leur habitat préférentiel, les dunes venant directement au contact du schorre. Les Amaurobius ont été signalés à plusieurs reprises dans des terrains halophiles; par exemple Amaurobius ferox dans l'estuaire du Frémur par Cazalet (1935); Amaurobius erberi est cité de Camargue par Schachter (1950) «sous des pierres, en station sèche, ce qui est normal pour cette espèce». Dans les schorres d'Arcachon, les Amaurobius voisinent fréquemment avec des pulmonés xérophiles (Helicella intersecta, Cocblicella acuta, voir ces espèces).

Dysderidae: Dysdera crocota C. L. Koch; cette espèce se rencontre fréquemment dans le même habitat que les Amaurobius; ses nids blancs, très serrés, sont tissés dans les anfractuosités des débris de maçonnerie, au-dessus ou au voisinage des plus hautes eaux. Les jeunes sont capturés au printemps mais, à Arcachon du moins, les adultes apparaissent dès le début de l'été (quelques captures datées même de mars). L'espèce est largement représentée tout autour de la baie, chaque fois qu'elle rencontre un biotope convenable (amas de pierres); je l'ai récoltée dans les mêmes conditions sur les bords du lac d'Hossegor. A Chaillevette (estuaire de la Seudre), elle est fréquente sur les digues qui entourent les claires à huîtres. Schachter (1950) la cite de Camargue «en station humide, mais aussi en station sèche ... elle n'a jamais été trouvée par nous près des eaux.»

Argiopidae: Argiope brünnichi Scop.; cette belle espèce est des plus communes sur l'Ile aux Oiseaux, ainsi que tout autour de la baie; elle tisse fréquemment ses toiles entre les buissons de Suadea fruticosa; adultes et cocons se récoltent à la fin de l'été (septembre). Non caractéristique des régions littorales, où elle été cependant signalée à plusieurs reprises (JACQUET 1949, dans la Manche, BAUDOIN 1961, dans les schorres de Charente).

Araneus cornutus CLERCK; mêtme localisation et mêmes remarques que pour l'espèce précédente. 


\section{Arachnides Opilions}

Phalangium parietinum DE GEER; commun dans tous les schorres explorés.

\section{Arachnides Acariens}

Les récoltes confiées à divers spécialistes sont en cours de détermination. Outre Bdella vulgaris, déjà signalé dans la haute slikke où sa présence est plus constante, le sol du Puccinellietum abrite de nombreux acariens (Thrombidiidés, Oribatidés, Gamasidés, Ixodidés), parmi lesquels Hermannia sp. (peut-être Hermannia bistriata Nrc. signalé dans l'estuaire du Frémur par CazAlet 1935), qui pullule en été sous les laisses à dessication lente, au niveau d'Alexia myosotis.

\section{Myriapodes}

Les myriapodes ne contribuent que faiblement au peuplement de la zone intertidale; on connaît cependant quelques exemples d'espèces qui s'aventurent assez loin le long de la grève et dont l'habitat exclusivement littoral, permet de les considérer comme de véritables halobies; c'est à ce groupe que se rattachent les espèces citées de la haute slikke: Bracbyschendyla monodi et Geopbilus algarum decipiens. Les espèces des schorres ou de la zone parhalienne, sont moins étroitement attachées aux bords de mers; on y rencontre soit des formes faiblement halophiles, soit des haloxènes.

\section{Diplopodes}

Cylindroiulus frisius (VERHOEFF); est une espèce consideree comme halophile par Scrubart (1929), parfois très abondante sous les hautes laisses du fond de la baie d'Arcachon; ainsi dans la région de Lège Arès, Cylindroiulus frisius pullule sous les laisses de Juncus et de roseaux accumulés au pied des digues, en compagnie des isopodes terrestres, d'Orchestia gammarella et d'Alexia myosotis. Cylindroinlus frisius est beaucoup moins abondant sous les laisses de zostères du Puccinellietum; les peuplements qu'il constitue se dissocient alors de la faune halobie; par exemple, sur I'lle aux Oiseaux, les talitridés abondent sous les laisses ou les épaves qui ceinturent le Truc Vert, tandis que Cylindroiulus frisius se réfugie plus haut, parmi les pierrailles accumulées sur le tertre; en hiver, au moment des très fortes marées, l'ensemble de la faune se regroupe, Orchestia gammarella et Porcellio scaber rejoignant alors Cylindroiulus frisius dans son habitat.

Glomeris marginata VILLERs; assez commun en bordure de la zone parhalienne, notamment sous les laisses fluviales de l'estuaire de la Leyre; s'aventure plus rarement sous les hautes laisses du Puccinellietum (Mestras). 


\section{Chilopodes}

Litbobius sp.; divers Lithobius (tricuspis MeINERT, forficatus L.) se rencontrent cà et là en bordure de la zone parhalienne, sous les débris végétaux accumulés, à la recherche de la petite faune qui s'abrite dans le même habitat.

Pachymerium ferrugineum (C. $\mathrm{KOCH}$ ); est un halophile commun sur le littoral; Caussanel (1962) l'a signalé dans la région d'Arcachon, sous les bois échoués des plages océaniques, au niveau des hautes mers de vive eau, en compagnic de divers coléoptères et de l'amphipode Orchestia microphtalma Amanieu \& Salvat; l'espèce est plus rare sous les laisses du schorre où je l'ai cependant récoltée tant sur l'Ile aux Oiseaux qu'à Mestras et dans le Juncetum de Lège. Pachymerium fermuginenm a fait l'objet d'une monographie écologique de Palmen \& Rantala (1954).

\section{Isopodes marins}

Sphaeroma rugicauda LEACH; espèce à large répartition, dans toute l'étendue des plages abritées et saumâtres. Pullule dans le schorre, soit en pleine eau dans les flaques temporaires à salinité variable (avec Palaemonetes varians), soit sous les épaves, même en position très haute, avec Orchestia gammarella, Alexia myosotis, divers coléoptères et araignées.

\section{Isopodes terrestres}

Tylidae: Tylos latreillei Audoun; espèce liée aux plages sableuses océaniques, accidentelle sur les plages abritées (Claouey, Lège).

Oniscidae: Halopbiloscia (Halophiloscia) couchi (KINAHAN); selon VANdel (1962) «cette espèce est un halophile typique; elle se rencontre au bord immédiat de la mer, dans l'étage supralittoral des écologistes, sous les pierres ou parmi les débris végétaux rejetés par la mer». Peres \& PiCARd (1964) la considèrent comme caractéristique de la Biocénose des laisses à dessication lente dans l'étage supralittoral. Halophiloscia couchi a été récolté à diverses reprises à Arcachon, sous les hautes laisses du Puccinellietum, avec Orchestia gammarella et Porcellio scaber, mais toujours beaucoup moins abondant que ces deux dernières espèces; en revanche il pullule au sommet de l'étage supralittoral à Guéthary (côte basque) sous les pierres, en bordure de la plage exposée (11 juin 1964, 5 mâles, 21 femelles gestantes, 5 femelles au repos sexuel).

Chaetophiloscia elongata (Dotrfus); récolté dans la baie d'Arcachon, sur l'lle aux Oiseaux, en décembre 1963 et février 1964, en compagnie de Metoponorthus sexfasciatus puis à Mestras en janvier 1965, avec Orchestia gammarella et des gastéropodes du genre Clausilia; laisses et terreau en bordure de la zone parhalienne; les récoltes ne comportaient que des femelles au repos génital.

Philoscia muscorum (SCOPOLI); espèce halophile signalé fréquemment dans les plages d'estuaires (Monod 1926, Lejoup \& Konietzko 1955, Salmon 1959); son habitat 
coïncide alors avec celui d'Alexia myosotis et le sommet des peuplements d'Orchestia gammarella. Philoscia muscorum occupe donc les biotopes qui, ailleurs, sont occupés, comme nous le verrons plus loin, par Porcellio scaber. C'est ainsi que je l'ai observé dans l'embouchure de la Slack à Wimereux (Amanieu 1962), à Roscoff sur la rive gauche de la Penzé, dans la baie de l'Aiguillon, dans l'estuaire de la Gironde et à Arès dans la baie d'Arcachon; toutefois, je n'ai que très rarement récolté ensemble Pbiloscia muscormm et Porcellio scaber, même lorsque les stations où se rencontrent chacune des deux espèces sont peu éloignées (par exemple à Arès); il est possible que la compétition biotique interdise la cohabitation.

Proportion des sexes et activité reproductrice: Sur 314 individus récoltés, 231 étaient des femelles et 83 des mâles, soit une proportion de $26,4 \%$ de mâles, un peu inférieure à la proportion de $30 \%$ indiquée par VANDEL, qui a observé 535 exemplaires. Dans les récoltes datées d'Arès, janvier 1964, toutes les femelles étaient au repos génital; dans les récoltes datées de Wimereux, mai 1961, 50\% des femelles étaient gestantes et $50 \%$ au repos; dans les récoltes datées de Roscoff, 2 juin 1964, il y avait 21 femelles au repos pour 52 femelles gestantes.

Porcellionidae: Metoponorthus (Polytretus) sexfasciatus Budde-Lund; selon VANDEL (1962) «en Europe cette espèce est strictement littorale, et ne pénètre jamais dans les montagnes ... lorsqu'on la rencontre à l'intérieur des terres (Digne, Toulouse, Paris), c'est toujours le fait d'importations accidentelles dues à l'homme». En ce qui concerne Metoponortbus (Polytretus) sexfasciatus sexfasciatus BuDde-Lund, seule sous espèce que l'on rencontre en France, «sur la côte atlantique, elle n'est connue que d'une seule station en France continentale: l'embouchure de l'Adour, près de Bayonne. Par contre, elle existe dans toutes les Iles du littoral atlantique français.» Cette espèce nous a en effet été rapportée de l'embouchure de l'Adour par M. LE CAMPION; en outre, nous l'avons récoltée à plusieurs reprises dans la baie d'Arcachon, sur l'lle aux Oiseaux, au sommet du Truc Vert, très peu au-dessus des stations de Porcellio scaber. Metoponorthus sexfasciatus s'abritait avec Chaetopbiloscia elongata et diverses araignées de la zone parhalienne (Amaurobius, Dysdera crocota) sous des pierres et des débris variés. Plus rarement, j'ai retrouvé Metoponortbus sexfasciatus le long des digues qui marquent la limite continentale du Puccinellietum, entre la Teste et le Teich. Cette espèce n'est jamais très abondante à Arcachon, en outre, très agile, elle échappe souvent à la capture et mes récoltes ont toujours été réduites à quelques individus.

Porcellio scaber scaber LATREILLE; espèce à large répartition qui s'est adaptée à des milieux très variés et dans laquelle se sont différenciées plusieurs variétés, plus étroitement liées à des biotopes particuliers. LEGRAND (1949) a signalé en Bretagne que «cette espèce pullule en bordure de la mer, à la limite des vagues dans les détritus, en compagnie d'Oniscus asellus et de Ligia oceanica». Porcellio scaber scaber est abondant sur le flanc des dunes marines qui bordent le sud de la baie d'Arcachon (pointe du Cap-Ferret, banc d'Arguin, dunes du Pyla); il est alors exclusivement représenté par la variété arenaria Dollfus (1892), d'un jaune sale; on le retrouve également le long des prés salés entre la Teste et le Teich, puis dans la région d'Andernos et d'Arès, enfin autour de l'lle aux Oiseaux; dans ces dernières stations c'est toujours la forme type qui est représentée. 
Porcellio scaber scaber forme type, est certainement l'isopode terrestre le plus abondant dans les schorres d'Arcachon; ses colonies, formées de plusieurs milliers d'individus, se rassemblent sous les laisses de haute mer; la strate colonisée par Porcellio scaber est de faible amplitude; localisé principalement au pied des digues qui marquent la limite continentale du Puccinellietum, il s'en éloigne volontiers vers l'aval, colonisant tout l'Obionetum tabulaire sur une profondeur de plusieurs centaines de mètres (Mestras), ce qui ne représente cependant qu'une dénivellation de 10 à $20 \mathrm{~cm}$, mais par contre ne grimpe sur les digues, au-dessus de $4,60 \mathrm{~m}$, que de manière tout à fait accidentelle. La variété arenaria colonise plus facilement les zones arides (flanc des dunes); dans les rares stations où la variété est susceptible de se rencontrer au voisinage du type (Lège), alors que le type s'arrête au pied du talus ou de la dune qui borde le schorre, la variété arenaria s'établit tonjours plus haut.

A Arcachon, Porcellio scaber forme type est associé à Orchestia gammarelle au plus haut niveau de son aire d'extension. A Mestras, l'isopode et l'amphipode constituent chacun, sous les laisses de zostères, des populations étroitement mélangées et d'une extraordinaire densité; les deux espèces s'entredévorent d'ailleurs fréquemment, à l'occasion des mues, sans qu'apparemment cela ait des conséquences favorables à la dominance de l'une ou de l'autre. Le troisième partenaire, tout aussi constant, est le gastéropode Alexia myosotis. J'ai retrouvé la même association à Porcellio scaber, Orchestia gammarella, Alexia myosotis, dans les environs de Roscoff, sous des pierres éboulées au pied de la falaise qui borde la rive gauche de l'Horn, non loin de son embouchure, dans la baie du Mont St-Michel, et à Guéthary (côte basque), également au pied de la falaise qui borde le sommet de la plage de galets. En d'autres cas, l'isopode se trouvait seulement en présence de l'un ou de l'autre des deux autres partenaires; par exemple, dans l'embouchure de la Canche près du Touquet, dans la baie d'Authie, dans la baie de l'Aiguillon (embouchure du Lay), dans diverses stations de l'estuaire de la Gironde et de la côte basque. L'association n'est cependant obligatoire pour aucune des trois espèces; il y a même une tendance à la dissociation des habitats, l'amphipode occupant la frange la plus basse, l'isopode occupant la frange la plus haute, le pulmoné étant localisé à la frange médiane, sur laquelle empiètent les deux autres partenaires; c'est le cas dans la baie d'Arcachon, sur certaines stations de l'lle aux Oiseaux; à Arès les trois espèces cohabitent mais non loin de là, à Lège, les stations sont nettement séparées. Outre les différences géographiques, s'ajoutent des fluctuations saisonnières qui conditionnent regroupement ou dissociation; par exemple, au Truc Vert, au moment des très fortes marées du début de l'hiver, Orchestia gammarella et Porcellio scaber se rassemblent au sommet de la butte, Alexia myosotis moins agile restant à son pied; mais en été, les trois espèces se regroupent sous les épaves échouées autour de la butte, dans le Puccinellietum. En défnitive, s'il est certain que principalement Orchestia gammarella et Porcellio scaber ont des exigences écologiques suffisamment voisines pour que leurs biotopes puissent coïncider, il ne s'établit cependant pas de liens systématiques d'interdépendance, les rapports éventuels étant limités à des confrontations occasionnelles entre prédateurs et proies, chacun pouvant être tour à tour, ou le prédateur ou la proie.

Armadillidiidae: Armadillidium album Dolıfus; commun sur les plages océaniques 
du littoral landais avec Tylos latreillei, accidentel sur les plages abritées. LEGRAND (1954) a souligné le caractère sabulicole et halo-hygrophile de cette espèce.

Armadillidium vulgare (LATREnLE); accidentel, rare, ne présente certainement pas d'affinités particulières avec les sites étudiés.

Armadillidium depressum BrandT; espèce haloxène de la zone parhalienne, assez commune sur les digues entre la Teste et le Teich mais abondante également dans les rues mêmes d'Arcachon.

\section{Crustacés Amphipodes}

Orchestia gammarella (Pallas); espèce déjà citée dans la haute slikke mais, comme nous l'indiquions, beaucoup plus caractéristique du schorre. Chevreux \& Fage (1925) la citent "Toutes nos côtes au dessus des pleines mers de vive eau; dans la terre humide, souvent loin de la mer, au bord des ruisseaux». Les migrations de diffusion sont surtout liées au rythme des marées; en été lors des marées de morte eau Orchestia gammarella s'étend sur tout le schorre; lors du retour des vives eaux l'espèce reflue avec le front de la marée dans le schorre supérieur; lors des très fortes marées hivernales, elle envahit même la zone parhalienne; ainsi après les fortes marées du début décembre 1963, les populations d'Orchestia gammarella du Puccinellietum sud de l'lle aux Oiseaux étaient, en majeure partie, groupées au sommet de la butte du Truc Vert, avec divers myriapodes, araignées, isopodes et gastropodes parhaliens. Enfin, les différentes classes d'âge d'une même population d'Orchestia gammarella ne se répartissent pas de manière homogène; les jeunes recherchent les abris meubles (laisses de zostères peu épaisses et desséchées par exemple), les adultes davantage les abris stables et peut être à climat plus humide.

Orchestia mediterranea A. CosTA; espèce de la haute slikke, accidentelle dans le schorre inférieur.

Talitrus saltator (MonT.); espèce des plages océaniques. Accidentelle dans les sables supralittoraux des plages abritées.

Talorchestia deshayesei (Audouin); espèce des laisses à dessication rapide du schorre. Accidentelle dans la haute slikke.

Talorchestia spinifera (E. Mateus); espèce des sables meubles de la haute slikke. Accidentelle dans le schorre. Etant donnée la faible abondance de cette espèce à Arcachon, il est difficile d'apprécier exactement sa position dans l'estran.

\section{Insectes Collemboles}

Anurida maritima (GuÉRIN); espèce de la haute slikke, accidentelle dans le Puccinellietum.

Archisotoma pulchella (Monrez); espèce accidentelle dans la haute slikte mais qui pullule par contre dans le Puccinellietum, particulièrement sous les hautes laisses à dessication lente. Halophile commun des côtes françaises de l'Atlantique. 
Axelsonia littoralis (MONIEz); largement réparti dans le Puccinellietum mais récolté toujours à un petit nombre d'exemplaires. Littoral atlantique et méditerranéen.

Isotomurus palustris (MÜLLER); récolté sous les laisses à dessication lente du Puccinellietum, avec l'espèce précédente et, plus fréquemment, notamment en hiver, sous les laisses à Juncus du Juncetum de Lège.

\section{Insectes Orthoptères et Dermaptères}

Parmi les Orthoptères et Dermaptères, peu d'espèces sont strictement inféodées aux bords de mers. A ma connaissance, on ne peut guère citer, parmi les premiers, que le petit grillon méditerranéen Mogoplistes squamiger (FrsH.), que l'on récolte «dans les rochers, sous les pierres et les algues, dans la zone battue par le mouvement des vagues» (ChOpARD 1951) et considéré par Soyer (1949), comme caractéristique, avec divers isopodes terrestres halophiles, de l'association à Critbmum maritimum, ainsi que Epacromius tergestinus (CHARP.) (= Aelopus tergestinus) espèce du littoral atlantique que l'on récolte notamment à Arcachon. Parmi les dermaptères, on citera Anisolabis maritima (Bonelli), espèce méditerranéenne que l'on récolte sous les galets et les débris littoraux et Labidura riparia (PALl.), espèce commune le long de nos plages, notamment sur tout le littoral landais.

De nombreux orthoptères creusent leurs nids ou déposent leurs oothèques dans la zone parhalienne; à la faveur de la faible amplitude des marées estivales, beaucoup d'entre eux envahissent le schorre supérieur, de juillet à septembre, ainsi qu'en témoignent les relevés de quelques chasses datant de l'été 1963. A l'exception d'Epacromius tergestinus et de Labidura riparia, toutes les espèces citées dans ce paragraphe doivent être considérées comme haloxènes.

\section{Orthoptères}

Manteidae: Empusa pennata (THuns.); mâles capturés de nuit au cours de chasses à la lumière dans le Puccinellietum de la Hume.

Mantis religiosa L.; commune partout en fin d'été, notamment dans le schorre supérieur de l'lle aux Oiseaux; forme verte exclusivement, alors que les individus bruns dominent dans la forêt landaise.

Conocephalidae: Conocephalus (Xiphidion) fuscus (F.); commun sur l'Ile aux Oiseaux et dans le Juncetum humide des bords du courant de Lège, en septembre.

Homorocorypbus nitidulus (Scor.) avec le précédent.

Tettigoniidae: Decticus verrucivorus (L.); cette belle espèce est abondante dans les dunes littorales, mais aussi dans le schorre supérieur de l'Ile aux Oiseaux et dans le Juncetum de Lège, au pied des dunes arides, entre Lillan et le courant de Lège.

Gampsocleis glabra (Herbst); dans le Puccinellietum de l'lle aux Oiseaux.

Platycleis affinis FIEBER; très commun avec le précédent. 
Tettigonia viridissima L.; particulièrement commun à la Hume et à Mestras, au voísinage des prairies continentales.

Ephippigeridae: Ephippiger ephippiger (FIEBIG); accidentel, avec le précédent.

Gryllidae: Acheta burdigalensis (LATR.); espèce recherchée intentionnellement et récoltée à deux reprises dans le Juncetum inondable de Lège Arès.

Gryllus campestris L.; commun en été dans tous les schorres supérieurs.

Pteronemobius beydeni (Fisch.); beaucoup plus précoce que les espèces précédentes capturé dans le Juncetum, en bordure du courant de Lège ainsi, que dans le Puccinellietum inférieur de Mestras.

Tetrigidae: Paratettix meridionalis (RAMB.); rares adultes capturés en hiver en bordure des esteys du Juncetum de Lège.

Tetrix subulata (L.); Egalement précoce, capturé à Lège et dans l'embouchure de la Leyre, dans les prairies humides à Scirpus.

Tetrix tenuicornis (SAHLB.); Puccinellietum de l'lle aux Oiseaux, autour du Truc Vert.

Catantopidae: Calliptamus italicus (L.); c'est le type même de l'espèce à large répartition qui pullule en été dans tous les schorres, comme dans toutes les prairies incultes; malgré son abondance, sa présence n'a aucun rapport avec l'originalité des prairies salées.

Acrididae: Acrotylus insubricus (Scop.); Lège et nord de l'Ile aux Oiseaux, accidentel.

Aiolopus strepens (LATR.); un peu partout, pas très fréquent, se remarque surtout par le fait qu'il est, avec Paratettix meridionalis, l'un des rares orthoptères que l'on peut capturer en hiver dans les prés salés.

Aiolopus thalassinus (F.); accidentel, deux captures à Arès.

Dociostaurus genei (Osck.); tout l'été, particulièrement dans le Juncetum de Lège, très actif par temps ensoleillé.

Epacromius tergestinus (CHARP.); considéré par CHOPARD (1951) comme «assez rare dans les prairies au bord de la mer et dans les marais salants». Cuenot (1927) écrit à son sujet: "cet acridien est tout à fait spécial aux prairies situées au bord de la mer et aux marais salants (adultes de juillet à octobre); il abonde dans les prés salés de la Teste, à l'Ile aux Oiseaux où il domine les autres acridiens, au banc de Pineau où il est à peu près le seul orthoptère ...»; c'est sans doute le seul orthoptère de notre région que l'on puisse considérer réellement comme halophile; je l'ai récolté à plusieurs reprises dans divers Puccinellietum (Ile aux Oiseaux, la Hume, Mestras) mais il n'abonde nulle part; sur l'Ile aux Oiseaux il n'est certainement pas dominant parmi les autres acridiens.

Locusta migratoria L. ph. danica Krrby; la forme solitaire du criquet migrateur n'est pas rare dans notre région, particulièrement en été, dans le schorre aride de Lège, assez en amont, à la lisière de la forêt.

Oedaleus decorus (GERM.); commun partout en été. 
Oedipoda coerulescens (L.), Oedipoda germanica (LATr.); les deux espèces sont communes, particulièrement la seconde qui s'avance très profondément en aval dans le schorre de l'Ile aux Oiseaux et qu'il n'est pas rare de capturer en été au niveau de la haute slikke.

Paracinema tricolor bisignata (Charp.); belle espèce commune en été dans le Puccinellietum de l'Ile aux Oiseaux, autour du Truc Vert.

Spbingonotus coerulans (L.); commun dans tous les schorres.

Stenobothrus lineatus (PanZ.); commun dans tous les schorres.

\section{Dermaptères}

Labiduridae: Labidura riparia (PALl.); espèce halobie, commune sur les plages océaniques le long du littoral landais; accidentelle sur les plages abritées de l'Ile aux Oiseaux.

Forficulidae: Forficula auricularia LINNÉ; espèce ubiquiste, particulièrement abondante dans les gravats de la zone parhalienne; en été, elle s'aventure sous les hautes laisses du schorre tant à Mestras qu'à Lège et sur l'Ile aux Oiseaux, avec les isopodes terrestres halophiles.

\section{Insectes Coléoptères}

Les coléoptères du Puccinellietum sont plus abondants et plus variés que ceux de la haute slikke; à côte d'halophiles plus ou moins strictement inféodés à ce biotope, on récolte des espèces à large répartition et dont la présence est sans rapport avec la proximité de la mer. Sans prétendre donner une liste exhaustive, nous nous efforcerons, par des exemples, d'indiquer les types principaux de peuplements de coléoptères qui occupent les schorres: les halobies du sol, les phytophages liés à des halophytes, les halophiles, les ubiquistes.

(1) Les ha lobies du sol. Staphylinidae: Cafius xantboloma Gravenhorst; est une espèce dont l'attachement aux bords de mer a été signalé depuis longtemps. Caussanel (1962), a bien précisé les conditions de son habitat à Arcachon; Cafius xantholoma pullule principalement sous les laisses de mer, en bordure des plages océaniques; sa densité peut y atteindre 200 individus au mètre carré. Sa présence sous les débris végétaux des plages abritées, n'est cependant pas à exclure; je l'ai récolté, rarement abondant mais présent avec régularité, sur l'lle aux Oiseaux, à la Hume, à Mestras, à Arès. Il cohabite sous les laisses de vive eau du schorre avec divers autres coléoptères (Dichirotrichus, Pogonus, etc.) ainsi qu'avec l'isopode Porcellio scaber, l'amphipode Orchestia gammarella, le pulmoné Alexia myosotis.

Ortbidus cribratus ERICHSON; non signalée sur les plages océaniques de la région par Caussanel (1962), cette espèce constitue au contraire l'un des éléments les plus constants de la faune des plages abritées; même habitat que la précédente, plus abondante sans cependant jamais pulluler. 
Trechidae: Emphanes normannus DEJEAN; est un petit carabique que l'on récolte fréquemment avec les espèces précédentes; je l'ai observé en outre dans le domaine de Certes, sur le sol très salé d'un ancien réservoir désaffecté, entièrement asséché (8 aout 1963), où il pullulait.

Pogonus chalceus Marsham; est un halobie de la plate forme du schorre, où il s'abrite çà et là, sous toutes les épaves de bonne dimension (planches, pierres, etc....); souvent avec les précédents mais se déplace plus activement et s'aventure non seulement sur toute l'étendue du schorre, mais aussi sur la haute slikke, particulièrement en été, aux marées basses de la première moitié de la nuit; je l'y ai rencontré souvent en incursion nutritielle, à la recherche de proies variées.

Harpalidae. Parmi les Harpalidae du schorre, seuls Dichirotrichus gustavii Crotch, ( $=$ D. pubescens Paxkull) et Dichirotrichus obsoletus DeJEAN, sont des halobies. Les Dichirotrichus sont caractéristiques du Puccinellietum (avec Porcellio scaber, Orchestia gammarella, Alexia myosotis, etc.) sur lequel ils circulent régulièrement mais, contrairement à Pogonus chalceus, ils ne semblent pas s'aventurer sur la haute slikke.

(2) Les phytophages liés aux halophytes. Curculionidae: De nombreux Curculionidae phytophages sont strictement inféodés à des halophytes; on trouvera dans le travail de Tempere (1934), une bonne liste des espèces de la région d'Arcachon; je me bornerai à citer ici deux exemples caractéristiques, tirés de captures faites dans le Puccinellietum.

Apion limonii KIRBY; pullule au printemps et au début de l'été non seulement sur divers Statice mais aussi sur Plantago maritima.

Psendopbytobius acalloides FAIRMAIR; est inféodé aux Suaeda. WAGNER (1937) a décrit une sous espèce temperei récoltée à Arcachon (Ile aux Oiseaux) par TEMPERE \& Agnus. Selon 'Tempere (1950) il s'agirait non pas d'une forme locale, mais d'une race atlantique de l'espèce qui était jusqu'alors considérée comme méditerranéenne. Le même auteur précise en outre l'éthologie de cette espèce inféodée à Suaeda fruticosa, halophyte de la marge continentale du Puccinellietum. Tempere a pu élever Pseudopbytobius acalloides s. sp. temperei à partir de très jeunes larves: «l'évolution complète, qui se poursuit chez nous en mai juin, demande de cinq à sept semaines, dont douze à quinze jours pour la larve et trois à quatre semaines pour les stades passés à l'intérieur d'une coque ... la larve attaque plusieurs feuilles et les vide successivement de leur parenchyme. Il semble bien n'y avoir qu'une seule génération annuelle... les imagos qui sont abondants surtout au début de juillet, attendent le printemps suivant pour s'accoupler et pondre». J'ai retrouvé Pseudophytobius acalloides s. sp. temperei à plusieurs reprises sur l'Ile aux Oiseaux et à Mestras.

(3) Les halophiles. Au voisinage des espèces que nous venons de citer, qui sont des hôtes exclusifs des bords de mers ou des sols salés continentaux, on trouve un certain nombre d'espèces plus fréquentes dans ces mêmes biotopes, sans que cependant leur présence en dehors d'eux soit à exclure.

Chrysomelidae: Cassida vittata VILLERs; est parfois très abondant aux pieds des 
Atriplex et de Spergularia marginata; ce double victus sur des Salsolacées et des Caryophyllacées, autrefois noté par Perris, a été confirmé par TEMPERE (1928).

Crepidodera impressa FaBricius; est un halophile que l'on récolte en abondance au début de l'été, en battant les Statice et divers halophytes du Puccinellietum.

Elateridae: Agriotes sordidus Illiger, Agriotes ustulatus Schall; à ma connaissance il ne s'agit pas d'espèces habituellement considérées comme halophiles; les récoltes permanentes que j'en ai faites sous les laisses de vive eau du schorre, me conduisent à penser que leur présence régulière dans un biotope aussi caractéristique n'est pas purement accidentelle, et qu'elle traduit une certaine halophilie.

Coccinellidae: Coccinella undecimpunctata LinNÉ; est en revanche un halophile classique dont la répartition, selon Sarnte-Claire Deville (1935), inclut «tout le littoral et régions salinifères de Lorraine et du Puy de Dôme; rare à l'intérieur». Je l'ai obtenu en abondance en battant les phanérogammes du schorre.

Staphylinidae: Staphylinus ater Gravenhorst; est, selon Sainte-Claire Deville (1935), réparti dans «toute la France; plus commun dans les régions maritimes»; il s'agit en effet d'un halophile que j'ai récolté non seulement à Arcachon, mais encore dans tous les estuaires de notre littoral atlantique que j'ai prospectés. Cette espèce ne descend pas au-dessous des hautes laisses de vive eau.

A ces halophiles ou halobies du schorre, on pourrait ajouter un bon nombre d'espèces de la plage océanique, qui s'installent occasionnellement sur la plage abritée, à l'occasion par exemple de la présence de la plante hôte. Ainsi la faune qui accompagne les Cakile maritima, étudiée par CAussaneL (1962), se retrouve éventuellement dans le schorre avec la plante hồte, mais il s'agit là d'observations accidentelles; en revanche, bon nombre d'espèces de la plage océanique manquent totalement sur la plage abritée, c'est le cas par exemple d'Eurynebria complanata (L.) absent même des schorres les plus sablonneux.

(4) L es ubiquis te s. Par ubiquistes nous entendrons d'une part les espèces qui fréquentent des biotopes variés (Littré: «il est ubiquiste se dit d'un homme qui se trouve bien partout») d'autre part les espèces qui, liées à un biotope précis mais épars (bousiers, nécrophages), disposent de puissants moyens de dispersion leur assurant une large répartition (Littré: «il est ubiquiste ... se dit par exagération, d'un homme qui voyage très fréquemment, de sorte qu'il paraît être dans plusieurs endroits à la fois ... ). Le schorre est accueillant pour de nombreux ubiquistes qui ne sont ni halobies, ni même halophiles. Je ne donnerai pas une liste complète des espèces capturées, ce qui serait sans intérêt pour notre sujet, mais à titre d'exemple et pour montrer la variété de cette faune, rapporterai quelques comptes rendus de chasse.

Les floricoles: Les espèces suivantes ont été obtenues en battant les phanérogammes du schorre de Gujan-Mestras en juillet 1962: Rhagonycha fulva Scopoli (Cantharidae); Notoxus monoceros L. (Anticidae), Colotes maculatus LAporte et Dolichosoma lineare Rossi (Malachidae), Olibrus corticalis PANzer (Phalacridae), Coccinella septempunctata L., Titthaspis sedecimpunctata I., Thea vigintiduopunctata L. (Coccinellidae), Phaedon veronicae BED. (Chrysomelidae). 
Les necrophages: Profitant des mortes eaux, j’ai installé, le 9 août 1963, trois appâts d'une dizaine de kilos chacun, constitués par des viscères de requins; les appâts ont été déposés à même le sol dans la région de Meyran, le premier dans une prairie continentale, le second sur la plate forme du schorre, le troisième sur la slikke. Ces appâts furent régulièrement visités toutes les vingt quatre heures pendant quatre jours. Sous le troisième, déposé sur la slikke, je récoltai seulement quelques amphipodes et notai la présence de pontes de mouches qui d'ailleurs se desséchèrent rapidement sans se développer; sous le second, déposé dans le schorre, je récoltai quelques amphipodes (Orchestia gammarella) mais surtout de nombreux coléoptères: Creophilus maxillosus L., Thanatophilus rugosus L., divers Saprinus dont Saprinus semi punctatus F., S. detersus IlL., S. semistriatus Scriba, enfin une grande quantité de Dermestes frischi KUG. et de nombreuses pontes de mouches saprophages qui évoluèrent normalement. Sous le premier appât, outre les mêmes espèces que sous le second, je récoltai également Necrodes littoralis L.

Les erratiques: On récolte, à l'aspirateur à bouche, de nombreuses espèces dont la présence sur le schorre est accidentelle et sans rapport avec l'originalité de ce biotope; ainsi les Harpalidae, tels Harpalus rubripes Dufschmot, assez commun sous les planches en épave, souvent avec Harpalus affinis Schrank et Acupalpus brunneipes STURm; divers Anisodactylus (binotatus FAB. et poeciloides STEPH.) ou Amara (dont $A$. aenea DE GEER) ne constituent pas davantage des captures exceptionnelles. D'autres espèces viennent manifestement de biotopes voisins; ainsi les ténébrionides (Blaps lusitanica Herb. ou Crypticus quisquilius L.) qui sont communs au voisinage des villages de l'tle aux Oiseaux, ou encore les grands curculionides Brachyderes lusitanicus F. et Hylobius abietis L., espèces inféodées aux pins, qui ne sont pas rares dans le schorre de Lège, directement contigu à la forêt landaise. Divers staphylinides, tels Philonthus sp. et Quedius sp., se rencontrent çà et là, tandis que Paederus littoralis GRAv, espèce ripicole qui fréquente habituellement les bords du lac de Cazaux, est parfois abondant par époque, en bordure des slikkes marines de la baie d'Arcachon; sans doute s'agit $\mathrm{il}$, dans ce dernier cas, d'individus égarés au cours de leur migration.

\section{Insectes Hemiptères}

La faune hémiptérologique de la région d'Arcachon a fait autrefois l'objet de relevés importants (voir notamment les catalogues de Lambertie). Cependant, comme le souligne CARAyon (1949), auquel je renvoie pour les références bibliographiques, «La zone littorale proprement dite paraît avoir été plus négligée». Les schorres et la zone parhalienne renferment, à vrai dire, une majorité d'ubiquistes et peu d'espèces caractéristiques; sauf mention contraire, les espèces citées ici doivent donc être considérées comme haloxènes.

\section{Insectes Homoptères}

Cercopidae: Divers Cercopis (dont Cercopis sanguinolenta Scop.) et Apbrophora (dont Aphrophora corticea Ger.) qui pullulent dans les prairies de la zone parhalienne s'étendent largement en été dans le Puccinellietum et le Juncetum. 
Membracidae: Nous ferons les mêmes remarques pour Gargara genistae FABR. capturé à Mestras, à Lège et sur l'Tle aux Oiseaux.

Typhlocibidae: Les Baccharis halimifolia de Lège et de la Teste sont souvent envahis par de petits typhlocibides qui y pullulent; espèces non déterminées.

Jassidae: A côte des espèces précédentes qui sont banales, le petit Jassidae halobie Aphrodes limicola EDwards 1908, est incontestablement le représentant le plus dense, le plus constant, et le plus caractéristique des homoptères du Puccinellietum. Chaque année il pullule de juin à octobre, sous les hautes laisses à dessication lente des Puccinellietum de Mestras et de l'Ile aux Oiseaux. Récolté également mais moins abondant dans la région de Lège. Gravestein (1965) a récemment rappelé qu'il y avait bien lieu de séparer Aphrodes limicola EDwards 1908 d' Aphrodes albifrons (Linné 1758). Apbrodes limicola «is exclusively halobiont and is only found in the Puccinellietum».

\section{Insectes Hétéroptères}

CARAYON (1949) a donné une liste des hétéroptères des prés salés d'Arcachon. J'ai moi même récolté les espèces suivantes:

Saldidae: Les Saldidae sont des insectes généralement liés aux sols humides ou aux rivages, dont certains représentants, considérés comme halophiles, ont été signalés à un niveau correspondant à la haute slikke. Divers Salda sp. sont communs sur les bords du courant de Lège dont les rives sont inondés par la marée. Dufour (1853) signale trois Salda des prés salés d'Arcachon. CARA yon (1949) signale Saldula saltatoria (L.) et Chartoscirta cincta (HER. SCH.) des prairies à salicornes et à graminés de la baie.

Nabidae: Contrairement aux précédents, les Nabidae sont plutôt cantonnés aux buissons et aux prairies ensoleillées de la zone parhalienne. Nabis major Costa, Nabis lineatus DHLB., sont communs dans le schorre supérieur. CARAYoN cite également Nabis capsiformis (GERM.) capturé à la pointe de l'Aiguillon (Arcachon) «espèce cosmopolite, très commune en Afrique, mais fort rare en France».

Capsidae: Les Poeciloscytus pullulent fréquemment au pied des halophytes, particulièrement des chénopodiacés, mais davantage dans les dunes ou les lettes littorales que dans les schorres; j’y ai récolté toutefois Poeciloscytus cognatus FIEB., P. bolosericeus Hahn., P. unifasciatus FAB.

Phytocoris ulmi L., pullule en haut du Juncetum de Lège au voisinage de la forêt et dans le Puccinellietum supérieur de l'Ile aux Oiseaux.

Exolygus maritimus E. WAGN. a été capturé sur l'Ile aux Oiseaux (plage nord, zone parhalienne) et au Cap-Ferret.

Lygaeidae: Beaucoup d'espèces banales ou sans signification particulière, comme Lamprodema manrum (FIEB.) qui pullulait dans les Puccinellietum de l'lle aux Oiseaux et de Mestras en juillet août 1964, ou divers Aphanus sp. qui viennent des pins ou des genêts de la zone parhalienne. 
Ischnodemus sabuleti FaLl.; est un halophile lié surtout aux oyats (Ammophila arenaria) des dunes océaniques; mais l'espèce n'est cependant pas rare au pied de diverses graminées du schorre supérieur.

Coreidae: Camptotus lateralis GrRm., Stenocephalus agilis Scop., Syromastes marginatus L.; schorres de Lège et de l'lle aux Oiseaux.

Pentatomidae: La famille est abondamment représentée dans les schorres par des espèces qui proviennent pour la plupart de la zone parhalienne. Eurydema cognata FIEB., Geotomus punctulatus (CosTA), Odontoscelis dorsalis FAB. capturés à Lège et sur l'Ile aux Oiseaux, sont faiblement halophiles; Aelia acuminata (L.), Brachypelta aterrima FsT., Eusarcoris inconspicuus (H. S.), sont des ubiquistes qui pullulent régulièrement dans le Puccinellietum de la Hume. Beaucoup d'espèces, telles que Brachynema sp. ou Graphosoma italicum Mull., sont des formes sinon abondantes, au moins communes, mais qui n'ont aucune signification particulière.

Piesmidae: J’ai retrouvé Piesma quadrata (FIEB.), signalé par CARAYon (1949), assez commun au pied des Atriplex de l'Ile aux Oiseaux. L'espèce est considérée comme halophile par LINDBERG (1935).

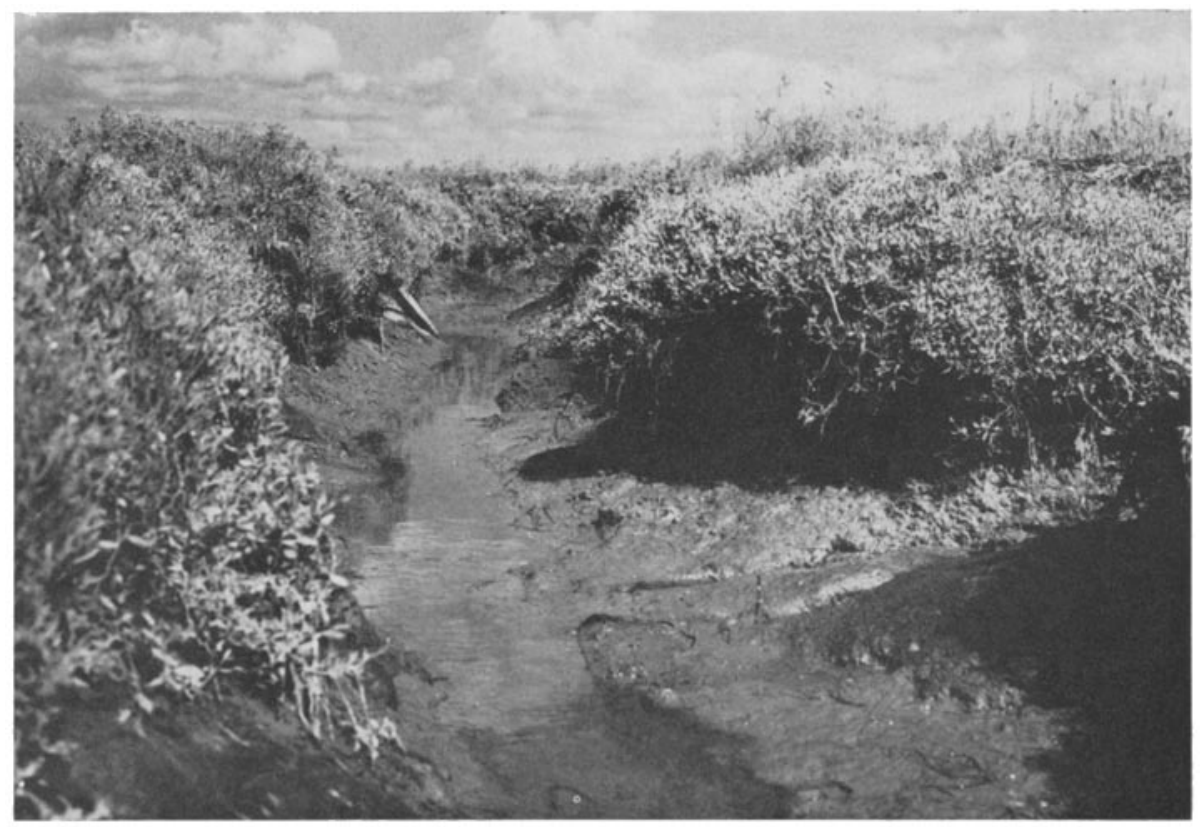

Fig. 18: Un estey dans le schorre supérieur. Le lic est étroit, encaissé entre des berges verticales, peuplé de vases molles à Nereis diversicolor. Obione portulacoides est particulièrement abondant sur les berges; sous l'abri de son feullage pullulent encore les talitridés de la haute slikke, Hyale nilssoni et Orchestia mediterranea; sur la paroi verticale des berges on retrouve la faune des microfalaises, notamment Paragnathia formica, divers Heterocerus, etc. 


\section{Remarques et conclusions sur la faune du schorre et de la zone parhalienne}

Si le schorre et la zone parhalienne constituent incontestablement deux biotopes distincts, les espèces qui les peuplent sont susceptibles de vagabonder de l'un à l'autre, soit de manière systematique, soit de manière accidentelle. Par exemple Orchestia gammarella, espèce qui vit habituellement sur le schorre, s'abrite dans la zone parhalienne au moment des très fortes marées hivernales. Au contraire divers insectes, par exemple les orthoptères, déposent leurs pontes dans la zone parhalienne, d'où ils envahissent le schorre durant leur vie libre. Ces restrictions doivent donc nuancer les expressions de «faune du schorre» ou «faune parhalienne» qui qualifient simplement un habitat préférentiel.

En ce qui concerne la macrofaune de l'édaphon, l'échantillonnage que nous avons réalisé est sinon complet, du moins bien représentatif des biotopes explorés. En ce qui concerne les espèces qui vivent non pas au contact même du sol mais au-dessus, par exemple tous les insectes floricoles munis de larges moyens de locomotion, notre échantillonnage, bien que très incomplet, est suffisant pour situer l'essentiel des problèmes; un recensement plus important sortirait du cadre de ce travail. Les peuplements du schorre sont remarquables par:

(1) L'absence quasi-totale de macrofaune endogée, caractère qui oppose fondamentalement le schorre à la slikke ou à la haute slikke. Les peuplements sont constitués par une épifaune vagile, souvent pourvue de larges moyens de locomotion, qui utilise le sol pour se déplacer et en explorer les abris. Très peu d'espèces, sinon de manière occasionnelle, n'y aménagent de galeries ou de terriers permanents; à ce point de vue, le sol du schorre diffère fondamentalement, pour l'écologiste, d'un sol meuble marin.

(2) La dominance des espèces littorales, dans la macrofaune vagile qui circule au contact mêrme du sol. Parmi ces espèces littorales, quelques unes sont d'origine marine (amphipodes talitridés, quelques isopodes et prosobranches), la plupart sont des halophiles d'origine continentale (par exemple les coléoptères du sol). Les araignées toutefois sont plutôt paludicoles que littorales.

(3) La dominance du peuplement d'origine continentale dans la macrofaune vagile qui vit non plus au contact du sol, mais au-dessus de celui-ci (insectes floricoles par exemple). Les formes littorales recensées dans cette strate, sont représentées uniquement par quelques phytophages liés à des halophytes (certains curculionides par exemple). Le schorre à ce point de vue, apparaît comme une prairie salée, dans laquelle le caractère prairial est plus important que le caractère halin..

(4) Comparativement à la slikke ou même à la haute slikke, on note dans le schorre que la part du peuplement d'origine marine (quelques amphipodes, isopodes et prosobranches) est faible, par rapport à la part d'origine continentale; ce caractère s'accrốt encore dans la zone parhalienne, où les espèces marines ne font que des incursions accidentelles.

(5) En définitive, le schorre représente une strate de convergence où, plus sans doute que nulle part ailleurs, les faunes littorales et continentales sont non pas confrontées mais étroitement imbriquées. 


\section{REMARQUES GENERALES SUR LES PLAGES ABRITEES}

\section{Classement des facies des plages abritées en fonction de leur coefficient d'assèchement}

La mesure de la teneur en eau des sédiments, à laquelle nous avons accordé une part importante, ne représente qu'une première approche de leur richesse en eau libre (eau de gravité), réellement disponible au profit de la faune. A une même teneur en eau brute, par exemple $30 \%$, correspond un sable saturé infralittoral, comme une argile supralittorale presque sèche. L'appréciation de l'eau disponible au point de vue écologique, doit donc tenir compte de la teneur en eau brute, rapportée à une certaine dimension caractéristique du sédiment, mesurant son aptitude à retenir une certaine quantité d'eau.

Bourcart \& Francis Boeuf (1942) on introduit la notion de teneur en eau sous tassement libre, dimension caractéristique du sédiment et indépendante des circonstances d'échantillonnage. La teneur en eau sous tassement libre cerne assez exactement la teneur en eau à saturation. Nous introduisons ici la notion de coefficient d'assèchement (CA) défini par la relation.

$$
\mathrm{CA}=\frac{\mathrm{T}-\mathrm{t}}{\mathrm{T}} \times 1000 \text { dans laquelle }
$$

$\mathrm{T}=$ teneur en eau sous tassement libre, est caractéristique du sédiment et indépendante des circonstances d'échantillonnage; $t=$ teneur en eau brute, au contraire est caractéristique à fois du sédiment et des circonstances d'échantillonnage. La différence $\mathrm{T}-t$, rapportée évidemment à $\mathrm{T}$, est en définitive caractéristique des circonstances, c'est à dire des faciès explorés, et relativement indépendante de la texture du sédiment. Le cacul du CA n'offre aucune difficulté pratique. Du point de vue méthodologique, cette notion paraît cependant assez abstraite et demande à être vérifiée par une estimation expérimentale.

Notons tout d'abord que pour un sol totalement $\sec (t=0) \mathrm{CA}=1000$, quelque soit le sol. Pour un sol saturé au contraire $(t=T), C A=0$. En août et septembre 1965, nous avons échantillonné 33 stations appartenant à douze des faciès précédemment décrits. (1) sur la slikke: Herbiers, sables à arénicoles, vases à Nereis, vases à Scrobicularia; (2) sur la haute slikke: sables argileux à Bledius spectabilis, sables vaseux à Coropbium volutator, sables poreux à Bledius arenarius, microfalaises; (3) sur le schorre: Puccinellietum et Obionetum; (4) dans la zone parhalienne: Agropyretum et base de talus parhaliens (au niveau de Beta maritima L.)

Chacun de ces faciès a été exploré sur l'Ile aux Oiseaux, à Mestras et à LègeArès. Chaque station comprenait 5 échantillons, prélevés en quinconce à $1 \mathrm{~m}$ les uns des autres, à pleine marée bassé et en vive eau. Les chiffres données concernent la moyenne de ces 5 échantillons regroupés par station. Les résultats apparaissent sur la Figure 19 et suggèrent les remarques suivantes.

La slikke est caractérisée par un CA dispersé mais en moyenne faible. Certains sables à arénicoles sont intégralement saturés d'eau (CA $=0$, alors que la teneur en eau brute des sables à arénicoles est particulièrement faible). Une seule mesure est quelque peu aberrante: Herbier de l'Ile aux Oiseaux (plage Nord très essorée) dans 


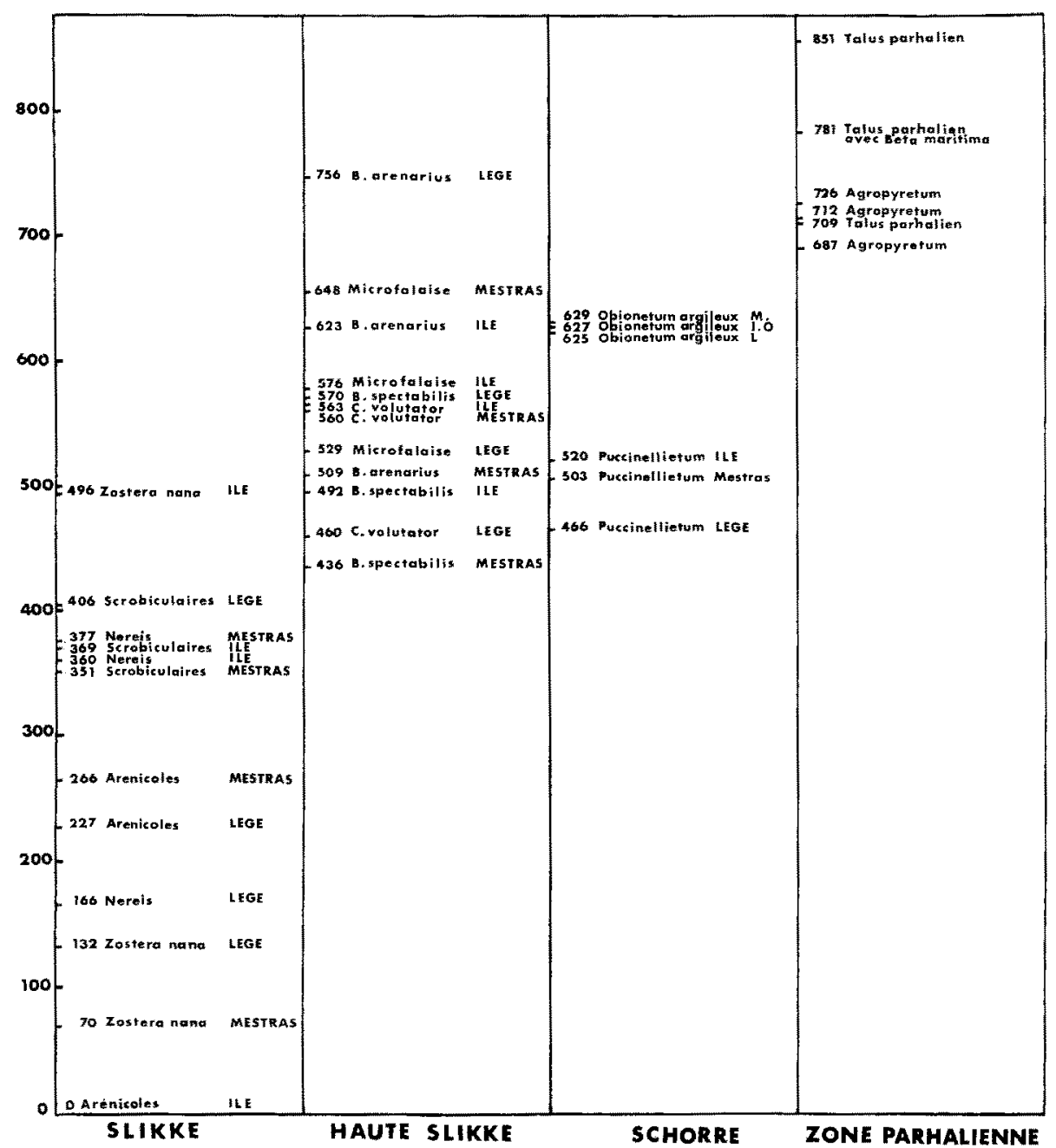

Fíg. 19: Distribution des coefficients d'assèchement de differents faciès des plages abritées de la région d'Arcachon

lequel $\mathrm{CA}=$ 496. Dans l'ensemble, les sédiments de la slikke, quelque soit le faciès, ont un CA faible, compris entre 400 et 0 , c'est à dire qu'ils sont saturés à 60-100\%.

La haute slikke est caractérisée par un CA moins dispersé et plus élevé que pour la slikke; là encore, une seule mesure est aberrante, un sable à Bledius arenarius de Lège où $\mathrm{CA}$ atteint 756. La majorité des mesures se situe entre 430 et 650 , c'est à dire que les faciès de la haute slikke sont saturés à $57-35 \%$.

Le schorre, contrairement à ce que nous attendions, n'apparaît pas comme différent de la haute slikke au point de vue du CA. Pourtant celà ne doit pas surprendre; les schorres explorés, argilo-sableux, ne s'assèchent que très peu entre deux vives eaux 
consécutives. Le revêtement phanérogamique, au lieu de contribuer à cet assèchement, assure au contraire une certaine protection. Or, contrairement à la haute slikke, le schorre n'abrite pas de faune endogée médiolittorale. C'est donc vraissemblabement la régularité des immersions de la haute slikke, et non sa teneur en eau, qui conditionne la position de la faune médiolittorale.

La zone parhalienne enfin, y compris l'Agropyretum, inclut des sols beaucoup plus secs dans lesquels le $C A$ varie de 680 à 850 (soit $15-32 \%$ de saturation).

Le CA mesure en définitive une dimension des sols liée à la fois à leur texture et à leur position dans l'estran; il permet un classement des faciès en accord avec les descriptions bionomiques et apparaît comme directement lié à la teneur en eau écolologiquement utilisable par la macrofaune.

\section{Correspondance des slikkes et schorres avec les étages intertidaux}

Nous avons rassemblé sur la Figure 20 la manière dont se répartissaient les différents types de faune infra, médio, supra et adlittorale, en précisant la correspondance avec slikke, haute slikke, schorre et zone parhalienne.

A la slikke, correspond une endofaune infralittorale, une épifaune médiolittorale.

A la haute slikke, correspond une endofaune médio-et supralittorale. L'épifaune, peu caractéristique, renferme des espèces vagiles en provenance soit du médiolittoral (certains amphipodes et gastéropodes) soit du supralittoral (insectes halophiles).

$\mathrm{Au}$ Puccinellietum correspond essentiellement une épifaune supralittorale; en hiver, cette épifaune fait des incursions dans l'adlittoral, alors qu'en été elle s'enrichit des incursions de la faune haloxène adlittorale.

A la zone parhalienne, correspond une épifaune, et éventuellement une endofaune estivale (fourmillières, terriers de lézards et de campagnols), adlittorales. Elle tolère en hiver les incursions de la fatne supralittorale du Puccinellietum.

Les notions de slikke et de schorre correspondent davantage aux données bionomiques, purement descriptives, alors que les notions d'étages tiennent plus étroitement compte des conditions écologiques; on ne peut en aucun cas conclure à une assimilation stricte des unes aux autres.

Les conditions d'humectation, fondamentalement différentes suivant que l'on considère la strate épigée ou endogée, conduisent à dissocier topographiquement l'épifaune et l'endofaune d'un même étage; par exemple, l'épifaune médiolittorale se situe dans les aires qui abritent une endofaune infralittorale. Il devient alors difficile de juger objectivement du déplacement vertical des étages, suivant que l'on a à faire à une plage de sable ou de vase. Par ailleurs, la limite des étages est incontestablement confuse sur une plage de vase et il est indispensable d'admettre des franges d'incursions très larges, dont nous avons à plusieurs reprises précisé l'amplitude. 


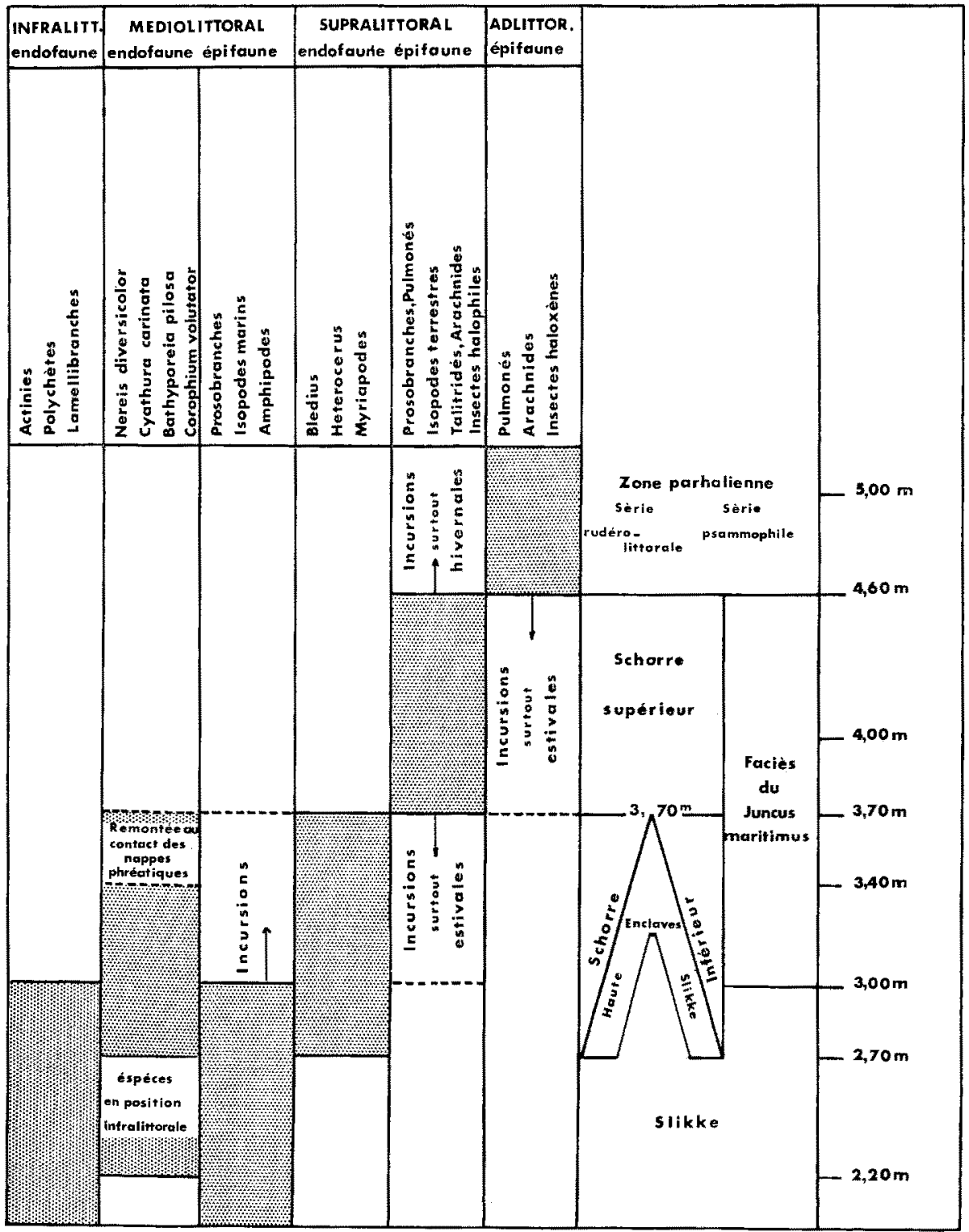

Fig. 20: Répartition de la faune des plages abritées de la baie d'Arcachon

Comparaison avec les plages semi-abritées

Salvat (1964) propose pour décrire la répartition de l'endofaune des plages meubles, d'adopter un cadre qui se réfère non plus aux niveaux marégraphiques, mais à quatre zones, dont la distinction repose sur les conditions hydrodynamiques 
interstitielles. Il reconnaît ainsi: (1) La zone de sable sec, exceptionnellement atteinte par l'eau de mer, (2) la zone de rétention, au-dessus de l'horizon de résurgence, (3) la zon de résurgence, saturée et soumise à un hydrodynamisme intense et (4) la zone de sa. ation, au dessous de l'horizon de résurgence. Quelques comparaisons, portant sur 2 ' ' espèces étudiées à la fois par SALvat $(1962,1964)$ et par nous mêmes sont figu. 'sur la Figure 21.

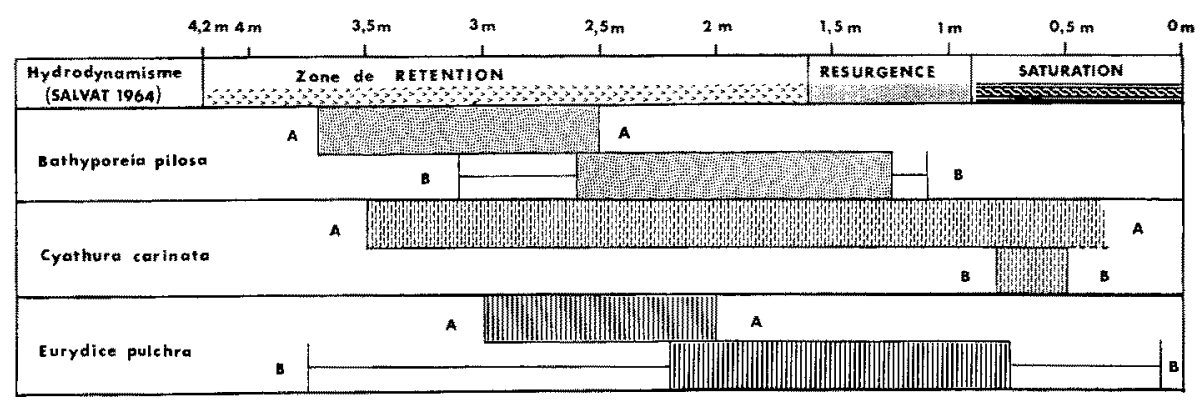

Fig. 21: Position de 3 espèces communes aux plages semi-abritées et abritćes de la région d'Arcachon. (AA) position dans la plage abritée. (BB) positions dans les plages semi-abritées d'après SALVAT $(1962,1964)$. En haut du graphique, hauteurs par rapport au 0 des cartes marines locales. Immédiatement au-dessous, position des 3 zones de Rétention, Résurgence et Saturation, définies par SALVAT (1964) dans les plages meubles semi-abritées d'Arcachon (La Vigne)

Eurydice puldbra est situé, selon Salvat (1964), en partie dans la zone de saturation, dans la zone de résurgence, et dans la zone de rétention; l'espèce s'étend de $+0,10 \mathrm{~m} \grave{a}+3,75 \mathrm{~m}$ environ, avec un niveau préférentiel entre $+0,75$ et $+2,20 \mathrm{~m}$ environ. Salvat (1961) considère Eurydice pulchra comme caractéristique du médiolittoral inférieur, J. P. LAgARdere (communication orale), sur les plages meubles très perméables des estrans battus de la côte océanique au sud d'Arcachon, récolte la même espèce au-dessous de l'horizon de résurgence. Sur les plages colmatées de l'Ile aux Oiseaux, Eurydice pulcbra se situe au contraire en position élevée, dans les sables saturés à Arenicola marina, entre +2 et $+3 \mathrm{~m}$, mais elle est localisée à la partie superficielle exondable de ces sables. Il y a donc déplacement vertical ascendant d'Eurydice pulchra, lorsque l'on va des plages perméables océaniques aux plages semi-abritées, puis réduction d'amplitude de la zone colonisée, lorsque l'on va des plages semi-abritées aux plages colmatées et imperméables des zones abritées. La position de cette espèce ne peut donc être considérée comme caractéristique d'un niveau marégraphique, mais davantage comme liée aux conditions hydrodynamiques de l'eau interstitielle.

Cyathura carinata «été récolté à la Vigne entre $0,48 \mathrm{~m}$ et $0,79 \mathrm{~m}$ dans un sédiment saturé d'eau» (SALVAT 1962). Sur l'Ile aux Oiseaux, l'espèce colonise les sables infralittoraux de la slikke et les sables médiolittoraux de la haute slikke, culminant à $+3,50 \mathrm{~m}$ avec un maximum de densité vers $+3,00 \mathrm{~m}$ il y a donc une augmentation considérable et difficilement explicable de la strate colonisée; ce résultat suggère 
que quelque autre facteur, peut être la salinité, limite l'extension de Cyatbura carinata à la Vigne.

Batbyporeia pilosa; selon Salvat (1964), cette espèce colonise principalement la zone de rétention et en partie la zone de résurgence. A la Vigne, plage perméable semi-abritée, elle s'étend entre $+1,10 \mathrm{~m}$ et $+3,10 \mathrm{~m}$ environ, avec un maximum de densité entre $+1,25 \mathrm{~m}$ et $+2,60 \mathrm{~m}$. Sur l'Ile aux Oiseaux Batbyporeia pilosa colonise les sables médiolittoraux exondables entre 2,50 et $3,50 \mathrm{~m}$. Il y a donc à la fois réduction d'amplitude et déplacement vertical ascendant de la zone colonisée. Si l'espèce se situe en position plus haute sur les plages abritées que sur les plages semiabritées, il y a cependant équivalence quant à l'hydrodynamisme des eaux interstitielles dans les deux habitats. Batbyporeia pilosa est lié aux sables poreux de la zone de rétention, reposant sur une assise saturée (zone de résurgence dans les plages semi-abritées, zone de saturation avec faible circulation dans les plages abritées).

\section{CONCLUSIONS GENERALES}

Le présent travail traite de la répartition et de l'écologie de la faune des plages abritées. Quatre grands ensembles sont identifiés, successivement étagés de bas en haut de la zone intertidale; la slikke, la haute slikke, le schorre et la zone parhalienne. Un rappel historique sur l'origine de ces termes permet d'en préciser la définition. Chacun de ces ensembles est ensuite étudié séparément quant aux peuplements qui les occupent, et aux structures édaphiques qui leur correspondent. Devant la diversité et l'étendue des plages abritées de la région d'Arcachon, il était nécessaire de fixer un cadre permettant de les décrire de manière cohérente. Parmi les différents cadres possibles, par exemple la localisation géographique, ou l'altitude moyenne, ou la variation de tel ou tel facteur abiotique (granulométrie, salinité etc.), nous avons opté pour celui intéresse le plus directement l'écologie, à savoir les peuplement eux-mêmes. Sur les plages abritées les peuplements sont monotones et se répartissent en mosaïque de divers faciès imbriqués, particulièrement dans la slikke et la haute slikke.

Quatre faciès fondamentaux sont reconnus dans les slikkes d'Arcachon. Le premier est celui des herbiers de Zostera nana; plus de 200 relevés de niveau permettent de préciser la position des herbiers qui culminent habituellement aux environs de la cote $+3 \mathrm{~m}$ (par rapport au zéro des cartes marines), soit au niveau des hautes mers des plus faibles marées. L'amplitude de l'herbier est cependant variable suivant les sites; des estimations chiffrées de manière précise, permettent d'apprécier raisonnablement l'extension ou la régression des herbiers en fonction de la structure des sols, de la pente des plages et de l'hydrodynamisme. Le faciès des herbiers de Zostera nana apparâ̂t comme hétérogène magré la monotonie du recouvrement phanérogamique; l'étude des cycles annuels de salinité dans les différentes strates du sol, fait ressortir les écarts importants qui opposent les herbier du secteur sud de la baie (Ile aux Oiseaux) à ceux du secteur nord (région de Claouey, Lège, Arès). Trois strates de peuplement sont reconnues: la macrofaune endogée, au contact des eaux interstitielles, trouve dans son habitat des conditions très différentes de celles auxquelles est confrontée la macrofaune épigée, au contact des eaux recouvrantes à marée haute; l'im- 
portance écologique de la couche de vase thixotropique, qui occupe la surface de l'herbier, est mise en évidence à propos de la faune dite de la pellicucle de surface, mais aussi par le rôle stabilisant qu'elle exerce sur l'eau interstitielle.

Le faciès des sables à arénicoles se caractérise essentiellement du point de vue abiotique par la perméabilité des sols qu'il occupe. Cette perméabilité, conséquence d'une porosité élevée, entraîne une meilleure oxygénation de la couche superficielle du sol qui, à marée basse, s'enrichit en air; cette aération superficielle oppose les sables à arénicoles aux autres faciès de la slikke. La situation topographique de ces sables, qui sont en fait des sables vaseux, leur teneur en eau libre élevée, le caractère infralittoral de la macrofaune endogée qui les occupe, confirment cependant leur rattachement aux faciès de la slikke.

Le faciès des vases à Nereis diversicolor est remarquable par la monotonie des peuplements qui l'occupent. L'étude des fluctuations de salinités montre que ces vases forment un ensemble cohérent, dont l'eau interstitielle évolue de manière largement indépendante de l'eau recouvrante. Par suite de leur mauvaise perméabilité, les vases à Nereis diversicolor sont difficilement imprégnées lorsqu'elles sont recouvertes par une eau peu salée, donc légère, alors que la densité élevée des eaux très salées permet au contraire leur intrusion; dans une certaine mesure ces vases «sélectionnent» donc en quelque sorte, les eaux sursalées qui se forment à leur surface à marée basse (par évaporation). Cette remarque explique que les vases à Nereis diversicolor se répartissent de manière préférentielle dans les plages presque horizontales et à hydrodynamisme faible, conditions qui favorisent la sédimentation fine et la formation, à marée basse, de flaques soumises à l'évaporation. Dans le Bassin d'Arcachon, le faciès des vases à Nereis diversicolor culmine à des niveaux assez variables, qui sont souvent au dessus de celui atteint par toutes les marées. Outre les faciès qu'il nomme, Nereis diversicolor envahit d'autres faciès, notamment de manière dense les vases à scrobiculaires et une partie du Juncetum de Lège; dans ce dernier habitat Nereis diversicolor a tendance à se situer en position médiolittorale.

Le faciès des vases à scrobiculaires est également étudié en fonction de sa répartition et des caractéristiques édaphiques qui lui correspondent. Ces vases se développent, avec leur peuplement caractéristique, dans les sites où les eaux souterraines renaissent en surface ou circulent à sa proximité immédiate. Contrairement aux vases à Nereis diversicolor, les vases à scrobiculaires sont donc dessalées en profondeur; en outre les strates profondes du sol ont une granulométrie plus grossière que les strates superficielles, en rapport avec la circulation des eaux souterraines.

En définitive, la slikke apparaît comme un ensemble de plusieurs faciès imbriqués, dont la haute teneur en eau libre conditionne le caractère infralittoral de la faune endogée. Les faciès de la slikke culminent en général au niveau atteint par toutes les marées ou peu au-dessus de ce niveau. Ils ont une extension inégale: le faciès de la Zostera nana est à la fois le plus étendu et le plus varié; il est habituellement surmonté soit par les sables à arénicoles, dominants dans le secteur sud de la baie, soit par les vases à Nereis diversicolor, dominants dans le secteur nord; plus rarement les herbiers de petite zostère sont directement confrontés aux faciès de la haute slikke. Quant au faciès des vases à scrobiculaires, il occupe le plus souvent des 
aires étroitement localisées, encaissements d'esteys, taches de résurgence, auxquelles correspondent des remontées d'eau douce.

La haute slikke surmonte topographiquement la slikke. Nous y avons reconnu cinq faciès: le faciès des sables argileux à Bledius spectabilis, le faciès des sables vaseux à Coropbium volutator, le faciès des sables poreux à Bledius arenarius et Bathyporeia, les aires à Spartina maritima et les microfalaises.

Les trois premiers faciès sont caractérisés par les peuplements de l'endofaune, représentée par des espèces vagiles qui aménagent des terriers stables envahis par l'air, au moins à marée basse; à marée haute en revanche, ces terriers tolèrent (Corophium volutator) ou non (Bledius spectabilis) l'inondation. Cette différence tient non pas à la position ou à la structure des sols occupés, mais au comportement caractéristique de chaque espèce; les peuplements zoologiques de la haute slikke sont donc considérés comme supralittoraux (insectes) ou médiolittoraux (amphipodes). L'importance quantitative de la macrofaune endogée est un trait qui rapproche la haute slikke de la slikke, alors que le comportement médio ou même supralittoral de cette faune annonce le schorre. JACQUET (1949) avait conçu la notion de haute slikke surtout à partir de considérations relatives à la morphologie du terrain et aux données marégraphiques; il nous est apparu qu'en outre, cette zone est occupée par une faune ayant un comportement écologique comparable, qui justifie de considérer la haute slikke comme un ensemble bionomique cohérent et bien individualisé. Il convenait de situer cet ensemble parmi ceux auxquels il est confronté. Le plus souvent, particulièrement dans la région d'Arcachon, les faciès zoologiques de la haute slikke sont dispersés en aires de petites dimensions et de faible amplitude verticale, dont les frontières s'établissent d'une part au contact du schorre, d'autre part au contact de tel ou tel faciès de la slikke. Ces aires sont généralement reliées par un faciès d'épiflore, celui de la Spartina maritima, qui en constitue en quelque sorte le lien morphologique; il est donc possible de préciser la position et l'amplitude de la haute slikke en nivellant les aires à recouvrement de spartines. Ces nivellements, auxquels nous avons consacré un long paragraphe, confirment que la haute slikke se situe du point de vue hypsométrique entre la slikke et le schorre; la haute slikke n'est donc inondée qu'à partir des marées moyennes; il en découle un cycle périodique de la teneur en eau des sols qu'elle occupe, teneur qui est plus élevée après une période de vive eau qu'après une période de morte eau; à ce point de vue, les sols de la haute slikke, qui ne sont jamais saturés par l'eau interstitielle, apparaissent donc comme très différents de ceux de la slikke. Les nivellements effectués permettent en outre de constater que, lorsque la morphologie du terrain retarde ou empêche l'écoulement normal de l'eau à marée basse, il y a non pas déplacement d'ensemble des faciès de la haute slikke, notamment du faciès des spartines, mais délamination partielle ou totale à partir de la base de ces faciès, au profit de l'extension de la slikke, généralement au profit des vases à Nereis diversicolor. Cette remarque permet de comprendre la dispersion des faciès de la haute slikke, leur faible amplitude, parfois même leur disparition, lorsque le schorre est directement confronté à la slikke. Comme l'avait pressenti Peres (1961), il n'y a pas, sur les plages de vase, transfert de la faune médiolittorale, mais amenuisement ou disparition de cette faune au profit d'une confrontation directe entre les peuplements supralittoraux du schorre et infralittoraux de la slikke. $\mathrm{Si}$, du triple point de vue morphologique, 
marégraphique et faunistique, la haute slikke apparaît donc comme une zone de transition d'amplitude variable, on y reconnaît cependant des peuplements homogènes et stables, qui justifient de la considérer comme un ensemble bionomique ayant une individualité propre. Enfin nous avons abordé le problème des microfalaises, accident morphologique très constant sur le type de plages étudiées ici. Les microfalaises sont surtout remarquables par la richesse et la densité de leur faune, non par sa spécificité.

En ce qui concerne le schorre, nous avons surtout étudié le Puccinellietum qui correspond dans notre région aux schorres littoraux de Verger (1956). Morphologiquement, le schorre apparaît comme une surface plane, il est souvent qualifié de schorre «tabulaire», ou en pente très douce. On peut parfois y discerner une partie inférieure, légèrement inclinée et plus humide, dans laquelle sont indentées les formations de la haute slikke, et une partie supérieure, presque horizontale, uniformément envahie par un recouvrement phanérogamique dense. Les phytosociologues ont été amenés à reconnaître dans ce recouvrement un «complexe en mosaïque» où s'imbriquent plusieurs faciès botaniques dont le plus répandu est celui de l'Obionetum. La marge continentale des schorres littoraux est marquée par un accident topographique, un talus à forte pente souvent aménagé en digue protectrice, généralement accompagné ou immédiatement précédé du faciès de l'Agropyretum, qui forme une ceinture caractéristique. Dans la région d'Arcachon, les schorres littoraux culminent aux environs de $+4,40$ à $+4,60 \mathrm{~m}$ au dessus du zéro des marées; ils ne sont inondés qu’aux marées de vive eau. Bien que très variée, la faune du Puccinellietum, présente des caractéristiques collectives qui l'opposent aussi bien à celle de la slikke qu'à celle de la haute slikke. Le Puccinellietum est dépourvu de macrofaune endogée dense, constituant des terriers ou des galeries permanents et stables; il est occupé par une épifaune vagile supralittorale qui circule au dessus du sol, à son contact ou dans ses abris (fissures, laisses, épaves). C'est à la mobilité de ses constituants que la faune du schorre doit son homogénéité. Contrairement à ce que nous avions noté pour les zones précédentes, il est difficile d'y reconnaître des faciès à peuplements monotones ayant une localisation spatiale précise; il y a donc, dans les mêmes territoires, confrontations de plusieurs types de faune, dont l'examen des coléoptères nous a permis de situer les exemples principaux. C'est ainsi qu'à côté d'halobies ou d'halophiles liés soit au sol, soit aux halophytes, on recense de nombreux ubiquistes n'ayant aucun rapport préférentiel avec le Puccinellietum considéré en tant que prairie salée. L'origine des peuplements est soit continentale (insectes, araignées, gastéropodes pulmonés etc.) soit marine (amphipodes, isopodes marins, mollusques prosobranches etc). «En définitive le schorre représente une strate de convergence ou, plus sans doute que nulle part ailleurs, les faunes littorales et continentales sont non pas confrontées mais étroitement imbriquées.»

En rapprochant les données relatives à la position topographiques du schorre, de celles concernant le rythme des marées, nous avons fait ressortir que, particulièrement au début de l'hiver, le Puccinellietum est soumis à des inondations fréquentes et parfois d'assez longue durée, alors que durant toute la belle saison il est hors d'atteinte de la marée. Cette remarque permet de préciser le rôle de la zone parhalienne par rapport aux peuplements du schorre. En hiver, la zone parhalienne, 
représentée par les flancs ou le sommet des talus qui ceinturent la marge continentale du schorre, apparaît comme un abri indispensable à la faune supralittorale du schorre qui vient s'y réfugier. En été au contraire, elle est le point de départ des éléments adlittoraux qui envahissent le Puccinellietum.

En conculusion de ce travail consacré à la bionomie des plages abritées, nous rattachons les notions de slikke, haute slikke, schorre et zone parhalienne, à l'étagement défini par le Colloque de Gènes. On ne peut conclure à une assimilation de ces deux notions. Alors que la première correspond mieux aux données purement descriptives et géographiques, la seconde introduit une interprétation éco-physiologique qui nécessite un examen distinct du comportement de chaque espèce.

\section{RESUME}

1. Le présent travail est consacré à l'étude écologique des plages abritées de la baie d'Arcachon (France). L'auteur tout d'abord, reconnaît et localise dans la région étudiée les quatres zones classiques qui partagent les estrans meubles abrités: slikke, haute slikke, schorre et zone parhalienne. Chacune d'entre elles est ensuite étudiée de manière plus détaillée, quant aux peuplements qui les occupent et aux structures édaphiques qui leur correspondent.

2. Dans la slikke, l'auteur, reconnaît et décrit quatre faciès fondamentaux: les herbiers de Zostera nana, les sables nus, type sables à arénicoles, les vases à Nereis diversicolor et les vases à scrobiculaires. Chacun de ces faciès est analysé quant à sa teneur en eau, porosité, salinité, pH et oxygène dissous, répartition hypsométrique. En ce qui concerne la faune de ces slikkes sablo-vaseuses, l'auteur souligne l'importance de la macrofaune endogée, son caractère infralittoral, la faible mobilité de ses représentants.

3. Dans la haute slik ke, l'auteur reconnaît et décrit cinq faciès: les sables argileux à Bledius spectabilis, les sables vaseux à Corophium volutator, les sables poreux à Bledius arenarius, les aires à Spartina maritima et les microfalaises. De nouveau, sont étudiés successivement les caractéristiques abiotiques, puis les peuplements de chaque faciès; l'auteur souligne l'action réciproque des structures édaphiques sur la répartition des peuplements, mais aussi des peuplements sur les structures édaphiques. Par exemple Corophium volutator et Bledius spectabilis édifient des galeries à parois stables, permanentes, quì ne peuvent être aménagées que dans certains types de sédiments; mais tandis que les galeries de B. spectabilis sont denses et étanches à la marée, celles de $C$. volutator sont plus dispersées et inondables; aussi la présence de l'insecte permet l'installation d'une faune d'accompagnement supralittorale, qui s'enterre au moment de la haute mer, celle de l'amphipode s'accompagne au contraire, d'une faune médiolittorale inondable.

4. Enfin, dans le $\mathrm{s} c \mathrm{~h}$ or $\mathrm{r}$ e et la $z$ on e parha li e n n e, l'auteur souligne l'absence de macrofaune endogée, la mobilité des peuplements vagiles épigés, leur balancement saisonnier lié au régime des marées. Il montre notamment qu'en été, le schorre abrite à la fois des espèces supralittorales marines ou halobies, et des espèces adlittorales haloxènes qui, venant de la zone parhalienne, sont attirées par la 
richesse trophique du Puccinellietum. En hiver, au contraire, la fréquence des immersions dans le schorre oblige la faune à refluer dans la zone parhalienne, où elle troure son abri.

5. Une classification des faciès étudiés en fonction de leur teneur en eau écologiquement utilisable par la macrofaune, un tableau de correspondance entre slikke, haute slikke, schorre et zone parhalienne d'une part, et étages intertidaux d'autre part, enfin une comparaison entre les peuplements des plages abritées et plages semi-abritées, complètent l'ensemble du travail.

\section{LITTERATURE CITEE}

Amanrev, M., 1962. Observations récentes sur les associations végétales et animales des terrains salés de l'embouchure de la Slack. P. v. Soc. linn. Bordeanx 99, 61-74.

- 1963. Evolution des populations de Paragnatbia formica (Hesse) au cours d'un cycle annuel. Bull. Inst. océanogr. Monaco 60 (1261), 1-12.

- 1966. Introduction à l'étude écologique des plages abritées et des étangs saumâtres du Bassin d'Arcachon. Act. Soc. linn. Bordeaux 103, 60 p.

- 1967. Introduction à l'étude écologique des réservoirs à poissons de la région d'Arcachon. Vie Milieu 18 B, 381-446.

- \& Cazaux, C., 1962. Animaux rares observés dans la région d'Arcachon en 1961-1962. P.w. Soc. linn. Bordeaux 99, 74-86.

- - 1963. Nouveaux animaux observés dans la région d'Arcachon en 1962-1963. P.-v. Soc. linn. Bordeaux 100, 163-173.

- - 1964. Nouveaux animaux récoltés dans la région d'Arcachon en 1964. P.-v. Soc. linn. Bordeaux 101, 1-11.

- \& Salyat, B., 1963a. Note sur la présence à Arcachon de deux Talitridae: Talorchestiat spinifera (E. Mateus 1962), Orchestia micropbtalma sp. 1. P.-v. Soc. linn. Bordeanx 100, $69-72$.

- - 1963b. Orchestia microphtalma. Description et affinités. Bull. Mus. Hist. nat. Paris 35, 302-310.

- - 1965. Sur Orchestoidea fischeri (H. MHLNE-Edwards, 1830), Talorchestia quadrispinosa Barnard, 1916 et Talorchestia spinifera (E. Mateus, 1962), Amphipodes Talitridae, Précisions systématiques et répartition géographiques. Crustaceana 8, 55-65.

Amoureux, L., 1963. Etude des teneurs en oxygène dans les eaux interstitielles de l'Aber de Roscoff. Cab. Biol. mar. 4, 23-32.

- 1966. Etude bionomique et écologique de quelques annélides polychètes des sables intertidaux des côtes ouest de la France. Thèse, Faculté des Sciences (C.N.R.S.), Paris, 218 pp.

Andre, M., 1939. Halacariens récoltés dans le bassin d'Arcachon en septembre 1938. Bull. Mus. Hist. nat. Paris 11, 118-122.

Baudorn, R, 1964. Titres et travaux scientifiques. Sedes, Paris, 77 pp.

Beauchamp, P. DE, 1914. Les grèves de Roscoff. Lhomme, Paris, $270 \mathrm{pp}$.

Bellan-Santini, D. \& PrCard J., 1963. Etude des variations au cours d'une année des amphipodes de quelques stations de fonds meubles dans la région de Marseille. Recl Trav. Stn mar. Endoume 43 (28), 21-32.

Berlan, L., 1932. Les Arachnides. Lechevalier, Paris, 485 pp.

Bertrand, H., 1939. Sur quelques crustacés malacostracés de la région dinardaise. Bull. Lab. marit. Dinard 20, 23-31.

Blois, J. C., Francaz, J. M., Gaudichon, S. \& Le Bris, L., 1961. Observations sur les herbiers à zostères de la région de Roscoff. Cab. Biol. mar. 2, 223-262.

Boisseau, J. P., 1962. Contribution à la faune du bassin d'Arcachon. Annélides, Sipunculiens, Echiuriens. P.-v. Soc. linn. Bordeaux 99, 113-126. 
Bouchet, J. M., 1962a. Etude préliminaire des conditions physiques et sédimentologiques d'un chenal du bassin d'Arcachon (chenal du Courbey). Bull. Inst. océanogr. Monaco 59 (1233), $1-19$.

- 1962b. Etude bionomique d'une fraction de chenal du bassin d'Arcachon (chenal du Courbey). Bull. Inst. océanogr. Monaco 59 (1252), 1-15.

Bourcart, J. \& Francis Boeuf, C., 1942. La vase. Actual. scient. ind. 927.

Brajnikov, B., Francis Boeuf, C. \& Romanowsky, V., 1943. Techniques d'étude des sédiments et des eaux qui leur sont associées. Actual. scient. ind. 952.

Brolemann, H. W., 1932. Myriapodes Chilopodes. Faune Fr. 25, 1-404.

- 1935. Myriapodes Diplopodes (Chilognathes I). Faune Fr. 29, 1-370.

Burmeister, F., 1939. Biologie, Okologie und Verbreitung der europäischen Käfer. Goedke \& Evers, Krefeld, 307 pp.

Callame, B., 1955. Sur la répartition de Arenicola marina L. dans les sédiments de la zone intercôtidale. C. r. bebd. Séanc. Acad. Sci., Paris 240, 1670-1672.

- 1960. Etude sur la diffusion des sels entre les eaux surnageantes et les eaux d'imbibition dans les sédiments marins littoraux. Bull. Inst. océanogr. Monaco 57 (1181), 1-18.

Carayon, J., 1949. Notes sur les hémiptères hétéroptères des environs d'Arcachon. Feuille Nat. 4, 15-20.

Caussanel, C., 1962. Recherches sur les coléoptères d'une plage de sable du littoral atlantique (Arcachon). D.E.S., Faculté des Sciences, Bordeaux.

Cazalet, M., 1935. Recherches bionomiques sur l'estuaire du Frémur. Bull. Soc, scient. Bretagne 12, 205-237.

Chevreux, E. \& Fage, L., 1925. Amphipodes. Faune Fr. 9, 1-488.

Chopard, L., 1951. Orthoptéroïdes. Fanne Fr. 56, 1-359.

CRAWFORD, G. I., 1937a. A review of the amphipod genus Corophium with notes on the British species. J. mar. biol. Assoc. U.K. 21, 589-630.

- 1937b. Notes on the distribution of burrowing isopodes and amphipods in various soils of the sea bottom near Plymouth. J. mar. biol. Ass. U.K. 21, 631-662.

CuenöT, L., 1927. Contributions à la faune du bassin d'Arcachon. 9. Revue générale de la faune et bibliographie. Bull. Stn biol. Arcacbon 24, 229-308.

Davant, P. \& Salvat, B., 1961. Recherches écologiques sur la macrofaune intercôtidale du bassin d'Arcachon. I. Le milieu physique. Vie Milieu 12, 405-471.

Debyser, J., 1957. La sédimentation dans le bassin d'Arcachon. Bull. Cent. Etud. Rech. scient., Biarritz 3, 405-418.

- 1961. Contribution à l'étude géochimique des vases marines. Institur Français du Pétrole, des Carburants et Lubrifiants, Rueil Malmaison, 249 pp. (Réf. 6005.)

Deguen, F. \& Molinter, R., 1961. Etudes écologiques et biocénotiques dans la baie du Brusc (Var). Les sols phanérogamiques. Bull. Inst. océanogr. Monaco 58 (1197), 1-50.

Delamare Debouttevmle, C., 1950. Collemboles marins de Banyuls. Vie Milieu 1, 100-101.

- 1953. Collemboles marins de la zone souterraine humide des sables littoraux. Vie Milieu 4, 190-319.

Demange, J. M., 1961. Sur deux myriapodes géophilomorphes des prés salés d'Arcachon. P.-v. Soc. linn. Bordeaux 99, 40-42.

Denis, J. R., 1924. Sur la faune française des Aptérygotes. 5e note. Bull. Soc. zool. Fr. 49, $554-586$.

- 1951. Captures d'araignées halophiles et hygrophiles. Bull. Soc. ent. Fr. 46, 147-152.

- 1952. Quelques araignées paludicoles de Gironde. Bull. Soc. ent. Fr. 47, 78-80.

Dessenorx, C., 1962. Contribution à l'étude de la faune des bois immergés du bassin d'Arcachon. D.E.S., Faculté des Sciences, Bordeaux.

Dufour, L., 1853. Excursion entomologique aux dunes de Biscarosse et d'Arcachon avec indication de quelques manoeuvres insecticeptologiques et réflexions. Act. Soc. linn. Bordeaux $19(9), 283-318$.

Fauver, P., 1923. Polychètes errantes. Faune Fr. 5, 1-488.

- 1927. Polychètes sédentaires. Faune Fr. 16, 1-494. 
FISCHER, E., 1928. Recherches de bionomie et d'océanographie littorales sur la Rance et le littoral de la Manche. Annls Inst. océanogr. 5, 201-429.

Fischer, P., 1865. Faune conchyliologique marine du département de la Gironde et des côtes du sud-ouest de la France. Act. Soc. linn. Bordeaux 25, 9-88.

- 1869. Faune conchyliologique marine... Suppl. 1. Act. Soc. linn. Bordeaux 27, 89-150.

- 1874. Faune conchyliologique marine ... Suppl. 2. Act. Soc. Linn. Bordeanx 29, 151-213.

Francis Boeuf, C., 1947a. Production et consommation d'oxygène par la pellicule superficielle des vases fluvio-marines. C. $r$. hebd. Séanc. Acad. Sci., Paris 225, 820-822.

- 1947b. Recherches sur le milieu fluvio-marin et les dépôts d'estuaire. Annls Inst. océanogr. 23, $149-344$.

FreEman, R. \& Rrgler, F. H., 1957. The responses of Scrobicularia plana (DA Costa) to osmotic pressure changes. J. mar. biol. Ass, U.K. 36, 553-567.

Grovannini, R., 1965. Revision des espèces benthiques méditerranéennes du genre Hyale. Recl Trav. Sin mar. Endoume 53 (37), 277-340.

Glemarec, M., 1964. Bionomie benthique de la partie orientale du Golfe du Morbihan. Cah. Biol. mar. 5, 33-96.

Gravestein, W. N., 1965. New faunistic records on Homoptera - Auchenorhyncha from the Netherlands North sea island Terschelling. Zool. Beitr. 11, 103-111.

Guérin, M., 1961. Etude de biotopes à Scrobicularia plana DA CostA. Cah. Biol. mar. 2, $417-436$.

Hart, T. J., 1930. Preliminary notes on the bionomics of the amphipod Corophium volutator Pallas. J. mar. biol. Ass. U.K. 16, 761-789.

Heydemann, B., 1960. Die biozönotische Entwicklung rom Vorland zum. Koog. 1. Spinnen (Araneae). Abh. math.-naturw. Kl. Akad. Wiss. Mainz 1960 (11). 745-913.

- 1962. Die biozönotische Entwicklung vom Vorland zum Koog. 2. Käfer (Coleoprera). Abb. math.-naturre. Kl. Akad. Wiss. Mainz 1962 (11), 771-964.

Howes, N. H., 1939. Observations on the biology and post-embryonic development of Idotea viridis (SLABBER) (Isopoda, Valvifera) from new England creek, south east Essex. I. mar. biol. Ass. U.K. 23, 279-310.

JACQUET, J., 1949. Recherches écologiques sur le littoral de la Manche. Les prés salés et la Spartine de Townsend. Les estuaires. La Tangue. Lechevalier, Paris, $374 \mathrm{pp}$.

Jeannel, R., 1941. Coléoptères Carabiques. Pr 1. Fanne Fr. 39, 1-571.

Joubin, L., 1894. Les némertiens. Soc. Ed. Sci., Paris, 235 pp. (Faune française).

KnÜlLE, W., 1959. Über italienische Arctosa-Arten (Aranae : Lycosidae). Archo zool. ital. 45, 253-270.

LAFON, M., 1953. Recherches sur les sables côtiers de la basse Normandie et sur quelques conditions de leur peuplement zoologique. Annls Inst. océanogr, 28, 113-161.

LEDoYer, M., 1962. Etude de la faune vagile des herbiers superficiels de Zostéracées et de quelques biotopes d'algues littorales. Recl Trav. Stn mar. Endonme 39 (25), 117-237.

- 1964. La faune vagile des herbiers de Zostera marina et de quelques biotopes d'algues infralittorales dans la zone intertidale en Manche et comparaison avec des milieux méditerranéens identiques. Recl Trav. Stn mar. Endoume 50 (34), 227-247.

Legrand, J. J., 1949. Contribution à l'étude des isopodes terrestres de la Bretagne. Bull. Soc. zool. Fr. 74, 53-67.

- 1954. Les isopodes terrestres du Poitou et du littoral charentais. Contribution à l'étude du peuplement atlantique. Mém. Mus. natn. Hist. nat. Paris (A) 6, 139-180.

Lezoup, E. \& Konietzko, B., 1956. Recherches biologiques sur les eaux saumâtres du BasEscaut. Mém. Inst. r. Sci. nat. Belg. 132, 1-100 5 pl.

Lemee, G., 1952. Végétation et écologie des tangues du havre de Portbail (Manche). Mem. Soc. bot. Fr. 1950/52, 156-165.

LindBERG, H., 1935. Halophile und halobionte Hemiptera. Tierwelt N.- u. Ostsee 11e 4, 103-124.

LUBET, P. E., 1956. Considérations écologiques sur les herbiers du bassin d'Arcachon. P.-v. Soc. linn. Bordeaux 96, 95-104. 
Manigault, P., 1932. L'effet de variations expérimentales de salinité, de température, de $\mathrm{pH}$., sur Littorina obtusata L. subsp. Littoralis Linné. Bull. Inst. oceanogr. Monaco 29 (605), 1-8.

Mars, P., 1961. Recherches sur quelques étangs du littoral méditerranéen français et sur leurs faunes malacologiques. Thèse. Faculté des Sciences, Paris. Vie Milieu (Suppl.) 20, 1-270.

Massart, J., 1907-1908. Essai de géographie botanique des districts littoraux et alluviaux de la Belgique. Recl Inst. bot. Léo-Errera 7 (1), 167-584; (2), 1-121.

- 1910. Esquisse de géographie botanique de la Belgique. Recl Inst. bot. Léo-Errera $7 \mathbf{b}$.

MatSAKIS, J., 1956. Développement postembryonnaire d'ldotea viridis (SLABBER) provenant de l'étang de Leucate. Vie Milieu 7, 287-300.

Molander, A. R., 1962. Studies on the fauna in the fjords of Bohuslän with reference to the distribution of different associations. Ark. Zool. 2, 15, 1 .

Monod, Th., 1926. La région de la basse Seulle. Etude bionomique. Trav. Stn biol. Roscoff 4, $2-74$.

Newell, R., 1962. Behavioural aspects of the ecology of Peringia (= Hydrobia) ulvae (PeNNANT) (Gasteropoda, Prosobranchia). Proc. zool. Soc. Lond. 138, 49-75.

- 1964. Some factors controlling the upstream distribution of Hydrobia ulvae (PenNant) (Gastropoda, Prosobranchia). Proc. zool. Soc. Lond. 142, 85-106.

Palmen, E. \& Rantala, M., 1954. On the life history and ecology of Pachymerium ferrugineum (C. L. KосH) (Chilopoda, Geophilidae). Annls zool. Soc. zool. bot. fenn. Vanamo 16 (3), 1-44.

Paulian, R., 1941. Les premiers étars des Staphylinoidea. Etude de morphologie comparée. Thèse, Faculté des Sciences, Paris.

Peres, J. M. \& Picard, J., 1955. Biotopes et biocoenoses de la Méditerranée occidentale comparés à ceux de la Manche et de l'Atlantique nord oriental. Archs Zool. exp. gén. 92, 1-70.

- - 1958. Manuel de bionomie benthique de la mer Méditerranée. Recl Trav. Stn mar Endoume 23 (14).

- - 1964. Nouveau manuel de bionomie benthique de la mer Méditerranée. Recl Trav. Stn mar. Endoume 47 (31), 1-137.

PiCard, J., 1965. Recherches qualitatives sur les biocoenoses marines des substrats meubles dragables de la région marseillaise. Thèse. Faculté des Sciences, Marseille, 160 pp.

PRUvot, G., 1897. Essai sur les fonds et la faune de la Manche occidentale (côtes de Bretagne) comparés à ceux du golfe du Lion. Archs Zool. exp. gén. 3 (5), 511-660.

Quignard, J. P., 1963. Remarques écologiques sur quelques Talitridae des étangs languedociens. Rapp. P.-v. Réun. Commn int. Explor. scient Mer Méditerr. 17 (3), 795-804.

Renaud-Debyser, J., 1963. Recherches écologiques sur la faune interstitielle des sables. Bassin. d'Arcachon, île de Bimini, Bahamas. Vie Miliew (Suppl.) 15, 1-157.

Rullier, F., 1959. Eude bionomique de l'Aber de Roscoff. Trav. Stn biol, Roscoff 10, 1-350.

SACCHI, C. F., 1954. Relations entre les groupements malacologiques terrestres et les conditions biogéographiques et biohistoriques des étangs roussillonnais. Vie Milieu 5, 529-564.

Sainte Clatre Deville, J., 1935-1938. Catalogue raisonné des coléoptères de France. Abeille, Paris $36(1-3), 1-467$.

Salmon, J., 1959. Contribution à la biologie des eaux saumâtres. Etude bionomique de la partie terminale de la rivière de l'Ic, à Binic (Côtes du nord). Bull. Soc. scient. Bretagne $34,81-120$.

Salvat, B., 1962. Faune des sédiments meubles intertidaux du Bassin d'Arcachon. Systématique et Ecologie. Tanaidacés, Isopodes, Amphipodes, Cab. Biol. mar, 3, 219-244.

- 1964. Les conditions hydrodynamiques interstitielles des sédiments meubles intertidaux et la répartition verticale de la faune endogée. C. r. bebd. Séanc. Acad. Sci., Paris 259, 1576-1579.

- 1967. La macrofaune carcinologique des sédiments meubles intertidaux (Tanaidacés, Isopodes et Amphipodes). Ethologie, bionomie et cycle biologique. Mém. Mus natn. Hist. nat., Paris (N.S.A. Zool.) 45, 1-275.

SChachiter, D., 1950. Contribution à l'étude écologique de la Camargue. Annls Inst. Oceanogr. $25,1-108$. 
Schubart, O., 1929. Thalassobionte und thalassophile Myriapoda. Tierwelt N.- u. Ostsee 11f, $1-20$.

Sollaud, E., 1925. Les associations végétales et animales des terrains salés de l'embouchure de la Slack. Trav. Stn zool. Wimereux 9, 212-232.

SoUTHWARD, A. J., 1953. The fauna of some sandy and muddy shores in the south of the Isle of Man. Proc. Trans. Liverpool biol. Soc. 59, 51-71.

SoYER, B., 1949. Etude statistique des groupements d’animaux dans les associations végétales des environs de Marseille. Bull. Soc, zool. Fr. 74, 117-121.

Spooner, G. M. \& Moore, Ph. D., 1940. The ecology of the Tamar estuary. VI. An account of the macrofauna of the intertidal muds. J. mar biol. Ass. U.K. 24, 283-330.

Strenzke, K., 1955. Collembola. Tierwelt N.- u. Ostsee 11f 2, 1-52.

TEMpere, G, 1928. Cassida vittata Vrulers (Coléoptères Chrysomelidae) et ses plantes nourricières. Act. Soc. linn. Bordeaux 80, 106-109.

- 1934. Nouveau coup d'oeil sur les Coléoptères des environs d'Arcachon et de la Teste. P.-v. Soc. linn. Bordeaux 1934, 1-20.

- 1950. L'Ethologie des Hypurini (Col. Curculionidae). Bull. Soc, ent. Fr. 55 (4), 57-61.

THorson, G., 1957. Bottom communities (sublittoral or shallow shelf). In: Treatise on marine ecology and paleoecology. Vol. 1. Ed. by J. W. Hedgpeth. Mem. geol. Soc. Am. 67 (1), $461-534$.

True Schlenz, R., 1965. Données sur les peuplements des sédiments à petites phanérogames marines (Zostera nana Roth et Cymodocea nodosa Ascher) comparés à ceux des habitats voisins dépourvus végétation (Côtes de Provence). Recl Trav. Stn mar Endoume 55 (39), 95-125.

Turmer, J. M., 1945. Végétation maritime du havre de Régnéville (Manche). Bull. Soc. bot. Fr. 92, 235-242.

- 1949. Végétation de la côte ouest du Cotentin. Mém. Mus. natn. Hist. nat., Paris 28, 1-72.

VADER, W. J. M., 1965. Intertidal distribution of Haustoriid amphipods in The Netherlands. Botanica gotboburg. 3, 233-246.

VANDEL, A., 1960. Isopodes terrestres. Pt 1. Fanne Fr. 64, 1-416.

- 1962. Isopodes terrestres. Pt 2. Faune Fr. 66, 1-931.

Verger, F., 1956. Quelques remarques sur la formation et le relief des schorres. Bull. Ass. Géogr. fr. 259/260, 146-156.

WATKIN, E. E., 1938. A revision of the amphipod genus Bathypareia Lindström. J. mar. biol. Ass. U.K. 23, 211-236.

WeILs, G. P., 1957. The life of the lugworm. New Biol. 22, 1-20.

Adresse de l'auteur: Dr. M. Amanieu

Institut de Biologie marine

Université de Bordeaux

(33) Arcachon, France 